\title{
The Gambia: Statistical Appendix
}

This Statistical Appendix for The Gambia was prepared by a staff team of the International Monetary Fund as background documentation for the periodic consultation with the member country. It is based on the information available at the time it was completed on July 7, 2005. The views expressed in this document are those of the staff team and do not necessarily reflect the views of the government of The Gambia or the Executive Board of the IMF.

The policy of publication of staff reports and other documents by the IMF allows for the deletion of market-sensitive information.

To assist the IMF in evaluating the publication policy, reader comments are invited and may be sent by e-mail to publicationpolicy@imf.org.

\author{
Copies of this report are available to the public from \\ International Monetary Fund $\bullet$ Publication Services \\ 700 19th Street, N.W. • Washington, D.C. 20431 \\ Telephone: (202) 6237430 • Telefax: (202) 6237201 \\ E-mail: publications@imf.org • Internet: http://www.imf.org \\ Price: $\$ 15.00$ a copy

\section{International Monetary Fund \\ Washington, D.C.}





\title{
INTERNATIONAL MONETARY FUND
}

\author{
THE GAMBIA
}

\section{Statistical Appendix}

Prepared by a staff team consisting of Mr. Calvin McDonald (Head), Mrs. Ruby Randall, Mr. Subramanian Sriram (all AFR) and Mr. Yi Wu (PDR)

Approved by the African Department

July 7, 2005

Contents Page

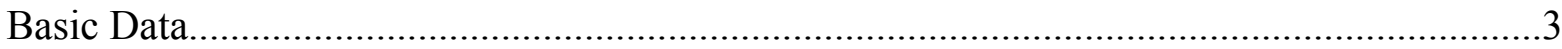

Statistical Appendix Tables

1. GDP by Sector at Constant Prices, 1998-2004 …….................................................

2. GDP by Sector at Current Market Prices, 1998-2004................................................6

3. GDP Growth by Sector at Constant Prices, 1998-2004 ………................................

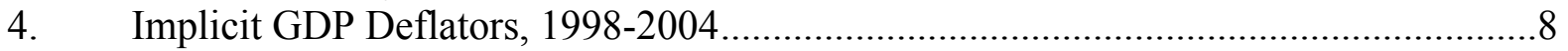

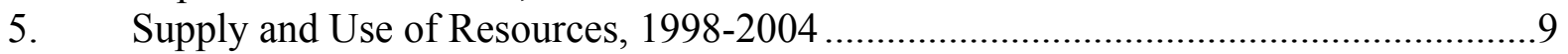

6. Savings-Investment Balance, 1998-2004 .............................................................10

7. Agricultural Production, 1998-2004 …………........................................................11

8. Minimum Producer Prices for Agricultural Commodities, 1998-2004 .......................12

9. Purchases and Disposition of Groundnuts, 1998-2004 .............................................13

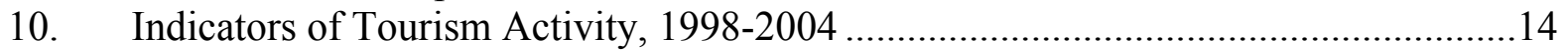

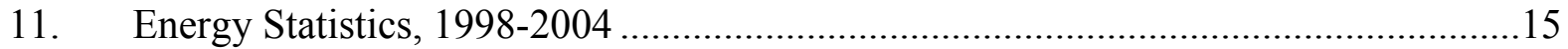

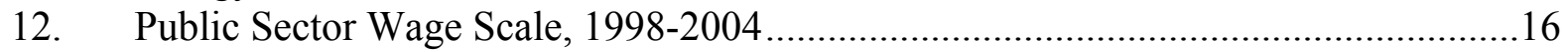

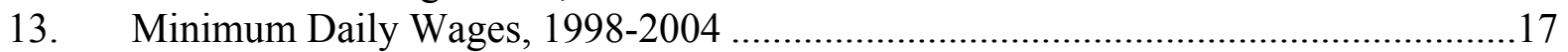

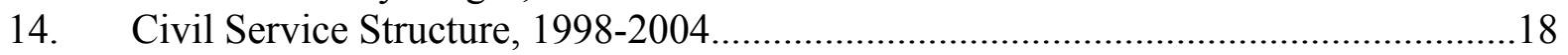

15. Private Employment by Industry, 1998-2004 _.........................................................19

16. Private Sector Employment by Size of Establishments and Industry, 1997-1998 …..20

17. Overall Consumer Price Index for Low-Income ……………...............................2

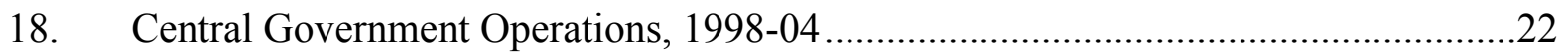

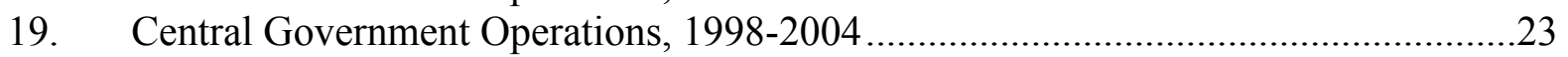

20. Functional Classification of Central Government Current Expenditure, 1998-2004 ..24

21. Central Government Social Expenditure and Social Indicators, 1998-2004 ...............25

22. Public Investment Program and Its Financing, 1998-2004.....................................26

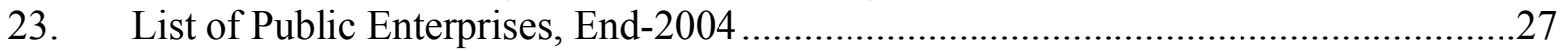

24. Monetary Survey, December 1998-March 2005 .....................................................22

25. Summary Accounts of the Central Bank, December 1998-March 2005 .....................29 
26. Summary Accounts of the Commercial Banks, December 1998-March 2005 ............30

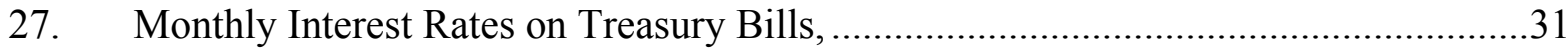

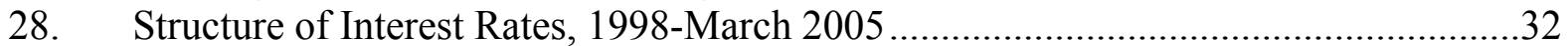

29. Distribution of Commercial Bank Credit by Sector, 1998-March 2005 ……...............33

30. Liquidity Position of Commercial Banks, 1998-March 2005 ......................................34

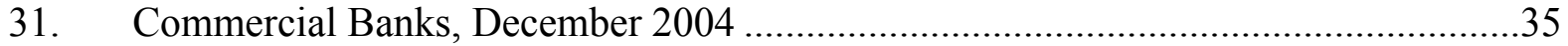

32. Distribution of Outstanding Government Securities, 1998-2005 ………...................36

33. Balance of Payments, 1998-2004 ………………..................................................

34. Composition of Merchandise Exports, 1998-2004 ….............................................38

35. Composition of Merchandise Imports, 1998-2004 ................................................39

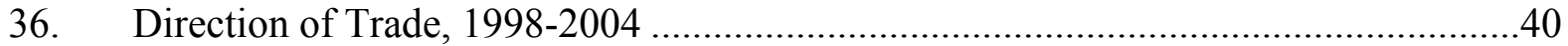

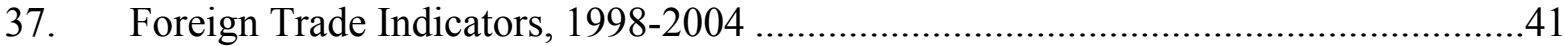

38. Public External Debt Outstanding and Debt Service, 1998-2004...............................42

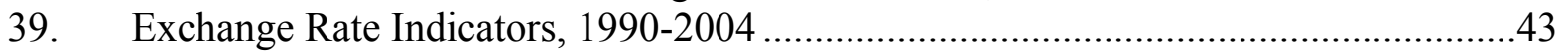

40. Interbank and Parallel Market Exchange Rates, 1998-2004 .......................................44

41. Summary of Tax System as of December 31, 2004...............................................45 
The Gambia: Basic Data

Area, population, and GNP per capita

$\begin{array}{lrl}\text { Area } & 10,700 \text { sq. km. } \\ \text { Population } & & \\ \text { Total (2003) } & 1.4 & \text { million } \\ \text { Growth rate (2000-2003; average) } & 2.8 & \text { percent } \\ \text { GDP per capita (2003) (staff est.) } & 256 & \text { US\$ }\end{array}$

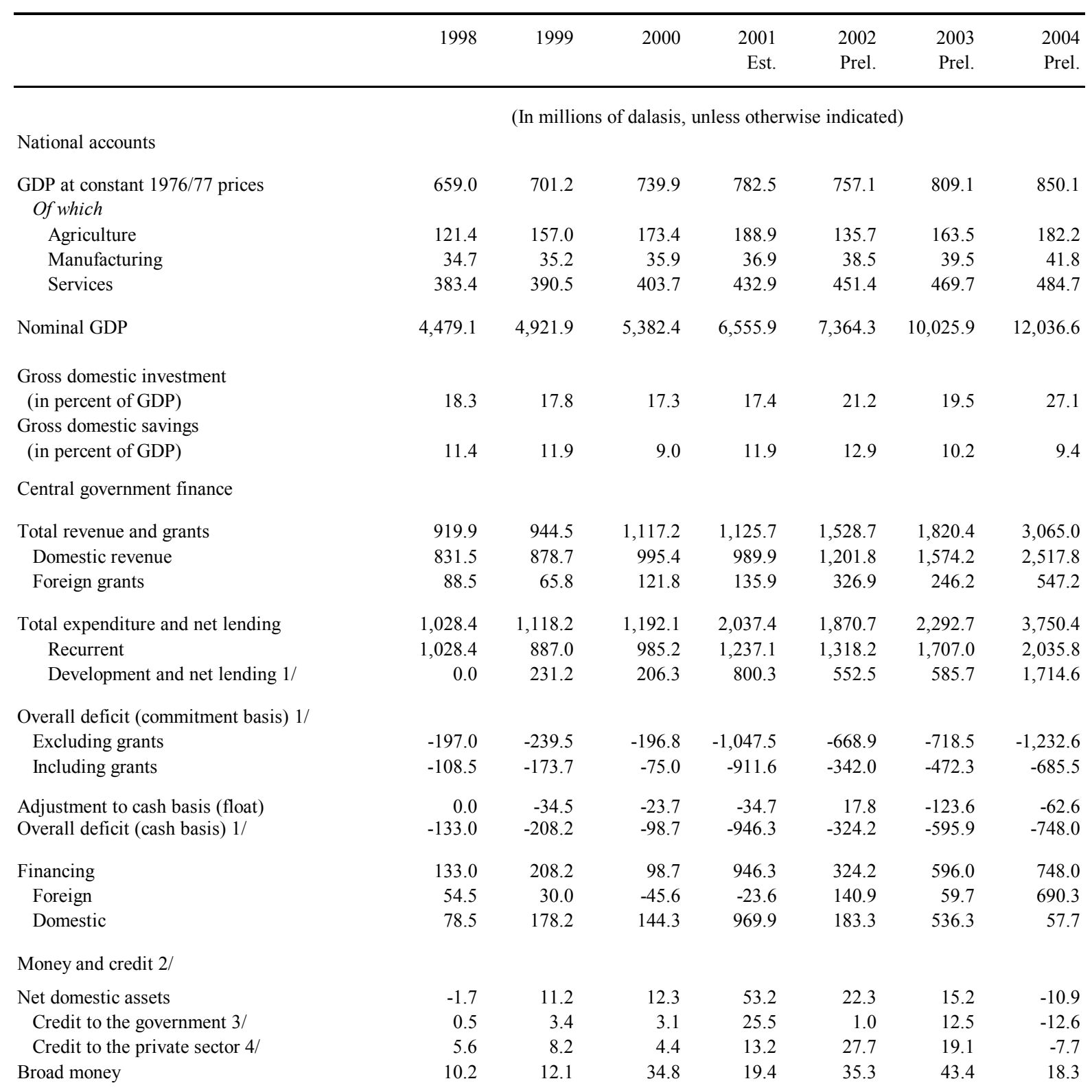


The Gambia: Basic Data (concluded)

\begin{tabular}{|c|c|c|c|c|c|c|c|}
\hline & 1998 & 1999 & 2000 & $\begin{array}{r}2001 \\
\text { Est. }\end{array}$ & $\begin{array}{l}2002 \\
\text { Prel. }\end{array}$ & $\begin{array}{l}2003 \\
\text { Prel. }\end{array}$ & $\begin{array}{r}2004 \\
\text { Prel. }\end{array}$ \\
\hline & \multicolumn{6}{|c|}{ (In millions of U.S dollars, unless otherwise indicated) } & \\
\hline \multicolumn{8}{|l|}{ Balance of payments } \\
\hline Trade balance & -78.9 & -68.7 & -63.3 & -50.0 & -62.4 & -60.0 & -108.4 \\
\hline Exports, f.o.b. & 130.4 & 120.2 & 126.6 & 102.1 & 109.3 & 101.0 & 127.0 \\
\hline Imports, c.i.f. & -209.3 & -188.9 & -189.9 & -152.1 & -171.6 & -161.0 & -235.4 \\
\hline Services (net) & 29.7 & 22.7 & 8.2 & 4.3 & 9.2 & 8.3 & 17.8 \\
\hline Transfers & 39.1 & 33.8 & 41.8 & 34.9 & 42.8 & 33.8 & 43.5 \\
\hline Private & 3.5 & 3.8 & 10.6 & 3.5 & 3.6 & 3.7 & 3.9 \\
\hline Official & 35.6 & 30.0 & 31.3 & 31.4 & 39.2 & 30.0 & 39.6 \\
\hline \multicolumn{8}{|l|}{ Current account balance } \\
\hline Excluding official transfers & -45.7 & -42.2 & -44.5 & -42.2 & -49.6 & -48.0 & -86.7 \\
\hline Including official transfers & -10.1 & -12.2 & -13.2 & -10.8 & -10.4 & -18.0 & -47.1 \\
\hline Capital account & 17.3 & 7.5 & 6.0 & 22.8 & 9.9 & 6.7 & 68.0 \\
\hline Official & 5.4 & 2.7 & 6.6 & 8.4 & 18.9 & 16.1 & 24.9 \\
\hline Private & 12.0 & 4.8 & -0.6 & 14.4 & -9.0 & -9.4 & 43.2 \\
\hline Unaccounted-for loss in reserves & 0.0 & 0.0 & 0.0 & -28.5 & 0.5 & 0.0 & 0.0 \\
\hline Errors and omissions & -0.8 & -0.6 & 17.4 & -39.6 & -0.1 & 6.3 & 12.6 \\
\hline Overall balance & 6.5 & -5.3 & 10.1 & -56.1 & -0.1 & -4.9 & 33.5 \\
\hline \multicolumn{8}{|c|}{ Current account balance (in percent of GDP) } \\
\hline Excluding official transfers & -10.9 & -9.8 & -10.6 & -10.1 & -13.4 & -13.6 & -21.6 \\
\hline Including official transfers & -2.4 & -2.8 & -3.1 & -2.6 & -2.8 & -5.1 & -11.8 \\
\hline \multicolumn{8}{|l|}{ Gross official reserves } \\
\hline In millions of SDRs & 102.0 & 98.0 & 111.4 & 63.0 & 67.2 & 62.3 & 84.6 \\
\hline In months of imports, c.i.f. & 5.8 & 6.2 & 7.0 & 5.0 & 4.5 & 4.4 & 4.1 \\
\hline \multicolumn{8}{|l|}{ External public debt } \\
\hline In millions of U.S. dollars & 460.5 & 451.5 & 454.3 & 458.3 & 481.1 & 511.6 & 580.7 \\
\hline In percent of GDP & 109.4 & 104.6 & 107.9 & 109.7 & 130.1 & 144.9 & 144.9 \\
\hline \multicolumn{8}{|c|}{ (In percent of exports and travel income) } \\
\hline \multicolumn{8}{|l|}{ Debt-service ratio 5/ } \\
\hline Including the Fund & 11.1 & 11.8 & 15.4 & 16.4 & 16.9 & 8.5 & 15.9 \\
\hline Excluding the Fund & 8.4 & 9.6 & 14.3 & 16.3 & 16.8 & 8.4 & 9.7 \\
\hline
\end{tabular}

1/ Data for 2001 include US\$28.5 million capital expenditure financed by a retroactive loan by the Central Bank of The Gambia (CBG) which the authorities indicated in October 2003 had not been recorded in official accounts.

$2 /$ In percent of broad money at the beginning of the period.

3/ Includes the central bank's foreign currency advance to the government in 2001.

4/ Includes public enterprises and central bank credit to foreign exchange bureaus.

5/ As a percentage of exports and travel income. After interim debt relief and HIPC grants. Excludes any accumulation of esternal arrears. 
Table 1. The Gambia: GDP by Sector at Constant Prices, 1998-2004

\begin{tabular}{|c|c|c|c|c|c|c|c|}
\hline & 1998 & 1999 & 2000 & 2001 & 2002 & 2003 & 2004 \\
\hline & \multicolumn{7}{|c|}{ (In millions of dalasis, at constant $1976 / 77$ prices, unless otherwise indicated) } \\
\hline Agriculture & 121.4 & 157.0 & 173.4 & 188.9 & 135.7 & 163.5 & 182.2 \\
\hline Groundnuts & 26.3 & 44.1 & 49.5 & 54.2 & 25.7 & 33.3 & 43.2 \\
\hline Other crops & 51.6 & 67.6 & 77.2 & 84.9 & 57.7 & 75.1 & 81.5 \\
\hline Livestock & 32.0 & 33.3 & 34.3 & 36.3 & 38.1 & 40.0 & 41.3 \\
\hline Forestry & 3.7 & 3.8 & 4.0 & 4.2 & 4.4 & 4.6 & 4.8 \\
\hline Fishing & 7.7 & 8.2 & 8.4 & 9.3 & 9.7 & 10.4 & 11.5 \\
\hline Industry & 70.2 & 71.8 & 75.5 & 80.1 & 88.0 & 93.6 & 96.8 \\
\hline Manufacturing & 34.7 & 35.2 & 35.9 & 36.9 & 38.5 & 39.5 & 41.8 \\
\hline Large and medium manufacturing & 23.5 & 23.8 & 24.3 & 25.0 & 26.3 & 26.8 & 28.4 \\
\hline Small manufacturing & 11.2 & 11.4 & 11.6 & 11.9 & 12.3 & 12.8 & 13.4 \\
\hline Construction and mining & 30.3 & 31.3 & 34.4 & 37.9 & 43.6 & 47.9 & 49.6 \\
\hline Electricity and water supply & 5.2 & 5.3 & 5.1 & 5.3 & 5.9 & 6.2 & 5.4 \\
\hline Services & 383.4 & 390.5 & 403.7 & 432.9 & 451.4 & 469.7 & 484.7 \\
\hline Trade & 79.5 & 75.9 & 81.1 & 87.0 & 90.6 & 91.0 & 91.3 \\
\hline Groundnuts & 9.7 & 10.0 & 11.2 & 12.9 & 13.6 & 14.0 & 14.3 \\
\hline Others & 69.8 & 65.9 & 69.9 & 74.0 & 77.0 & 77.0 & 77.0 \\
\hline Hotels and restaurants & 33.4 & 34.6 & 30.1 & 33.1 & 35.5 & 40.8 & 44.2 \\
\hline Transport and communications & 145.9 & 153.2 & 161.0 & 175.1 & 183.1 & 192.3 & 202.1 \\
\hline Transport & 64.2 & 64.4 & 66.8 & 71.4 & 74.3 & 78.0 & 80.3 \\
\hline Communications & 81.7 & 88.8 & 94.2 & 103.7 & 108.8 & 114.3 & 121.7 \\
\hline Real estate and business services & 38.8 & 39.0 & 39.8 & 41.8 & 43.5 & 44.8 & 45.8 \\
\hline Public administration & 67.5 & 69.4 & 72.6 & 76.2 & 78.5 & 80.1 & 80.1 \\
\hline Other services & 18.3 & 18.5 & 19.0 & 19.6 & 20.2 & 20.8 & 21.3 \\
\hline GDP at factor costs & 574.9 & 619.3 & 652.6 & 701.8 & 675.0 & 726.9 & 763.7 \\
\hline Indirect tax (net) & 84.1 & 81.9 & 87.4 & 80.7 & 82.1 & 82.3 & 86.4 \\
\hline GDP at constant market prices & 659.0 & 701.2 & 739.9 & 782.5 & 757.1 & 809.1 & 850.1 \\
\hline Real GDP growth (in percent) & 6.5 & 6.4 & 5.5 & 5.8 & -3.2 & 6.9 & 5.1 \\
\hline
\end{tabular}

Sources: Gambian authorities; and IMF staff estimates. 
Table 2. The Gambia: GDP by Sector at Current Market Prices, 1998-2004

\begin{tabular}{|c|c|c|c|c|c|c|c|}
\hline & 1998 & 1999 & 2000 & 2001 & 2002 & 2003 & 2004 \\
\hline & \multicolumn{7}{|c|}{ (In millions of dalasis, unless otherwise indicated) } \\
\hline Agriculture & $1,228.1$ & $1,493.3$ & $1,654.3$ & $2,042.3$ & $1,832.7$ & $2,822.4$ & $3,610.0$ \\
\hline Groundnuts & 236.2 & 347.1 & 389.7 & 464.7 & 284.5 & 634.1 & $1,066.6$ \\
\hline Other crops & 471.5 & 566.0 & 662.7 & 838.3 & 655.6 & $1,039.7$ & $1,230.6$ \\
\hline Livestock & 358.0 & 390.1 & 405.8 & 494.6 & 597.3 & 765.1 & 861.3 \\
\hline Forestry & 48.9 & 58.5 & 60.8 & 73.4 & 88.7 & 113.6 & 127.8 \\
\hline Fishing & 113.4 & 131.6 & 135.3 & 171.1 & 206.7 & 269.8 & 323.7 \\
\hline Industry & 507.1 & 545.4 & 584.4 & 736.8 & 964.4 & $1,274.2$ & $1,451.2$ \\
\hline Manufacturing & 219.0 & 233.0 & 244.8 & 301.6 & 378.2 & 485.4 & 582.8 \\
\hline Large and medium manufacturing & 146.0 & 155.3 & 163.2 & 201.7 & 254.1 & 324.0 & 390.2 \\
\hline Small manufacturing & 73.1 & 77.7 & 81.7 & 100.0 & 124.1 & 161.4 & 192.5 \\
\hline Construction and mining & 218.2 & 234.4 & 265.5 & 350.5 & 483.7 & 665.1 & 751.0 \\
\hline Electricity and water supply & 70.0 & 78.0 & 74.0 & 84.7 & 102.5 & 123.7 & 117.4 \\
\hline Services & $2,177.8$ & $2,311.4$ & $2,497.3$ & $3,174.0$ & $3,845.0$ & $4,989.7$ & $5,847.3$ \\
\hline Trade & 466.3 & 462.8 & 490.6 & 650.5 & 778.6 & $1,012.7$ & $1,151.3$ \\
\hline Groundnuts & 6.8 & 6.4 & 7.4 & 10.3 & 12.9 & 17.3 & 20.2 \\
\hline Others & 459.5 & 456.4 & 483.2 & 640.2 & 765.7 & 995.4 & $1,131.1$ \\
\hline Hotels and restaurants & 181.1 & 194.3 & 213.8 & 305.7 & 408.9 & 611.4 & 753.1 \\
\hline Transport and communications & 656.8 & 693.8 & 798.5 & $1,040.4$ & $1,279.8$ & $1,740.8$ & $2,073.9$ \\
\hline Transport & 336.9 & 364.1 & 378.7 & 486.3 & 581.6 & 824.4 & 964.8 \\
\hline Communications & 319.9 & 329.7 & 419.8 & 554.2 & 698.2 & 916.4 & $1,109.1$ \\
\hline Real estate and business services & 282.1 & 312.5 & 331.5 & 400.3 & 478.8 & 567.1 & 658.6 \\
\hline Public administration & 394.1 & 438.0 & 455.3 & 525.8 & 607.7 & 712.9 & 810.0 \\
\hline Other services & 197.5 & 210.0 & 207.6 & 251.2 & 291.1 & 344.8 & 400.5 \\
\hline GDP at factor costs & $3,913.1$ & $4,350.1$ & $4,736.1$ & $5,953.1$ & $6,642.1$ & $9,086.3$ & $10,908.5$ \\
\hline Indirect tax (net) & 566.0 & 571.8 & 646.3 & 602.8 & 722.2 & 939.7 & $1,128.1$ \\
\hline GDP at market prices & $4,479.1$ & $4,921.9$ & $5,382.4$ & $6,555.9$ & $7,364.3$ & $10,025.9$ & $12,036.6$ \\
\hline Nominal GDP growth (in percent) & 7.2 & 9.9 & 9.4 & 21.8 & 12.3 & 36.1 & 20.1 \\
\hline Real GDP growth (in percent) & 6.5 & 6.4 & 5.5 & 5.8 & -3.2 & 6.9 & 5.1 \\
\hline GDP deflator (in percent) & 0.6 & 3.3 & 3.6 & 15.2 & 16.1 & 27.4 & 14.3 \\
\hline CPI (annual average change, in percent) & 1.1 & 3.8 & 0.9 & 4.5 & 8.6 & 17.0 & 14.2 \\
\hline
\end{tabular}

Sources: Gambian authorities; and IMF staff estimates. 
Table 3. The Gambia: GDP Growth by Sector at Constant Prices, 1998-2004

\begin{tabular}{|c|c|c|c|c|c|c|c|}
\hline & 1998 & 1999 & 2000 & 2001 & 2002 & 2003 & 2004 \\
\hline & \multicolumn{7}{|c|}{ (Percentage changes) } \\
\hline Agriculture & -9.4 & 29.4 & 10.5 & 8.9 & -28.2 & 20.5 & 11.4 \\
\hline Groundnuts & -5.9 & 67.4 & 12.3 & 9.4 & -52.6 & 29.9 & 29.6 \\
\hline Other crops & -17.7 & 31.0 & 14.2 & 10.0 & -32.0 & 30.0 & 8.5 \\
\hline Livestock & 4.0 & 4.0 & 3.0 & 6.0 & 5.0 & 5.0 & 3.2 \\
\hline Forestry & 3.9 & 4.1 & 3.9 & 5.0 & 5.0 & 5.0 & 3.1 \\
\hline Fishing & -13.1 & 5.9 & 2.9 & 10.0 & 5.0 & 7.0 & 10.0 \\
\hline Industry & 5.7 & 2.3 & 5.1 & 6.1 & 9.8 & 6.4 & 3.3 \\
\hline Manufacturing & 2.4 & 1.4 & 2.0 & 2.7 & 4.5 & 2.6 & 5.7 \\
\hline Large and medium manufacturing & 2.6 & 1.3 & 2.0 & 3.0 & 5.0 & 2.0 & 6.0 \\
\hline Small manufacturing & 2.1 & 1.8 & 2.0 & 2.0 & 3.5 & 4.0 & 5.0 \\
\hline Construction and mining & 6.4 & 3.3 & 10.0 & 10.0 & 15.0 & 10.0 & 3.5 \\
\hline Electricity and water supply & 27.1 & 1.9 & -3.0 & 4.0 & 10.0 & 5.0 & -13.0 \\
\hline Services & 13.2 & 1.9 & 3.4 & 7.2 & 4.3 & 4.1 & 3.2 \\
\hline Trade & 8.9 & -4.5 & 6.8 & 7.2 & 4.1 & 0.4 & 0.4 \\
\hline Groundnuts & 6.7 & 3.1 & 12.3 & 15.0 & 5.0 & 3.0 & 2.5 \\
\hline Others & 9.2 & -5.5 & 6.0 & 6.0 & 4.0 & 0.0 & 0.0 \\
\hline Hotels and restaurants & 7.5 & 3.4 & -12.8 & 10.0 & 7.0 & 15.0 & 8.4 \\
\hline Transport and communications & 26.6 & 5.0 & 5.1 & 8.8 & 4.6 & 5.0 & 5.1 \\
\hline Transport & 7.0 & 0.3 & 4.0 & 7.0 & 4.0 & 5.0 & 3.0 \\
\hline Communications & 47.8 & 8.7 & 6.1 & 10.0 & 5.0 & 5.0 & 6.5 \\
\hline Real estate and business services & 1.1 & 0.4 & 2.2 & 5.0 & 4.0 & 3.0 & 2.2 \\
\hline Public administration & 7.0 & 2.8 & 4.6 & 5.0 & 3.0 & 2.0 & 0.0 \\
\hline Other services & 1.4 & 1.1 & 3.0 & 3.0 & 3.0 & 3.0 & 2.2 \\
\hline GDP at factor costs & 6.6 & 7.7 & 5.4 & 7.5 & -3.8 & 7.7 & 5.1 \\
\hline Indirect tax (net) & 5.6 & -2.6 & 6.7 & -7.7 & 1.7 & 0.2 & 5.1 \\
\hline GDP at constant market prices & 6.5 & 6.4 & 5.5 & 5.8 & -3.2 & 6.9 & 5.1 \\
\hline
\end{tabular}

Sources: Gambian authorities; and IMF staff estimates. 
Table 4. The Gambia: Implicit GDP Deflators, 1998-2004

(Base year, 1976/77=100)

\begin{tabular}{|c|c|c|c|c|c|c|c|}
\hline & 1998 & 1999 & 2000 & 2001 & 2002 & 2003 & 2004 \\
\hline Agriculture & $1,012.1$ & 951.3 & 954.1 & $1,081.3$ & $1,350.7$ & $1,726.2$ & $1,980.8$ \\
\hline Groundnuts & 897.2 & 787.6 & 787.4 & 858.2 & $1,109.2$ & $1,902.7$ & $2,469.2$ \\
\hline Other crops & 913.8 & 837.3 & 858.4 & 987.1 & $1,135.2$ & $1,385.0$ & $1,510.8$ \\
\hline Livestock & $1,119.2$ & $1,172.5$ & $1,184.1$ & $1,361.7$ & $1,565.9$ & $1,910.4$ & $2,084.0$ \\
\hline Forestry & $1,326.1$ & $1,523.4$ & $1,524.6$ & $1,753.3$ & $2,016.3$ & $2,459.9$ & $2,683.4$ \\
\hline Fishing & $1,465.3$ & $1,604.9$ & $1,603.4$ & $1,844.0$ & $2,120.5$ & $2,587.1$ & $2,822.2$ \\
\hline Industry & 722.4 & 759.6 & 774.3 & 920.1 & $1,096.4$ & $1,360.9$ & $1,500.6$ \\
\hline Manufacturing & 631.2 & 661.9 & 681.9 & 818.2 & 981.7 & $1,227.3$ & $1,394.5$ \\
\hline Large and medium manufacturing & 621.1 & 652.5 & 672.1 & 806.5 & 967.8 & $1,209.8$ & $1,374.7$ \\
\hline Small manufacturing & 652.3 & 681.6 & 702.3 & 842.7 & $1,011.3$ & $1,264.1$ & $1,436.4$ \\
\hline Construction and mining & 720.0 & 748.9 & 771.3 & 925.5 & $1,110.6$ & $1,388.3$ & $1,514.4$ \\
\hline Electricity and water supply & $1,345.3$ & $1,471.7$ & $1,439.9$ & $1,583.9$ & $1,742.3$ & $2,003.6$ & $2,185.7$ \\
\hline Services & 568.1 & 591.9 & 618.6 & 733.3 & 851.8 & $1,062.2$ & $1,206.4$ \\
\hline Trade & 586.9 & 609.7 & 605.1 & 748.1 & 859.8 & $1,113.3$ & $1,261.1$ \\
\hline Groundnuts & 69.9 & 64.0 & 66.3 & 79.6 & 95.5 & 124.1 & 141.1 \\
\hline Others & 658.8 & 692.6 & 691.7 & 864.6 & 994.3 & $1,292.6$ & $1,468.8$ \\
\hline Hotels and restaurants & 541.6 & 562.2 & 709.8 & 922.7 & $1,153.3$ & $1,499.3$ & $1,703.7$ \\
\hline Transport and communications & 450.2 & 452.9 & 496.0 & 594.2 & 698.9 & 905.3 & $1,026.6$ \\
\hline Transport & 524.7 & 565.4 & 567.3 & 680.8 & 782.9 & $1,056.9$ & $1,200.9$ \\
\hline Communications & 391.6 & 371.3 & 445.5 & 534.6 & 641.5 & 801.9 & 911.2 \\
\hline Real estate and business services & 726.9 & 802.1 & 832.4 & 957.3 & $1,100.9$ & $1,266.0$ & $1,438.6$ \\
\hline Public administration & 583.9 & 631.1 & 627.0 & 689.7 & 773.9 & 890.0 & $1,011.3$ \\
\hline Other services & $1,079.0$ & $1,135.1$ & $1,089.9$ & $1,280.6$ & $1,440.7$ & $1,656.8$ & $1,882.6$ \\
\hline GDP at factor costs & 680.6 & 702.4 & 725.8 & 848.3 & 984.0 & $1,250.1$ & $1,427.7$ \\
\hline Indirect tax (net) & 704.6 & 712.4 & 739.6 & 747.0 & 879.9 & $1,142.1$ & $1,277.6$ \\
\hline GDP at constant market prices & 679.7 & 701.9 & 727.4 & 837.8 & 972.7 & $1,239.1$ & $1,415.9$ \\
\hline
\end{tabular}

Sources: Gambian authorities; and IMF staff estimates. 
Table 5. The Gambia: Supply and Use of Resources, 1998-2004

\begin{tabular}{|c|c|c|c|c|c|c|c|}
\hline & 1998 & 1999 & 2000 & 2001 & 2002 & 2003 & 2004 \\
\hline & \multicolumn{7}{|c|}{ (In millions of dalasis) } \\
\hline Supply of resources & $7,063.9$ & $7,468.6$ & $8,241.8$ & $9,466.8$ & $11,372.4$ & $15,692.2$ & $20,227.2$ \\
\hline GDP at current prices & $4,479.1$ & $4,921.9$ & $5,382.4$ & $6,555.9$ & $7,364.3$ & $10,025.9$ & $12,036.6$ \\
\hline \multicolumn{8}{|l|}{ Imports of goods and } \\
\hline Use of resources & $7,063.9$ & $7,473.5$ & $8,241.7$ & $9,466.8$ & $11,372.4$ & $15,692.2$ & $20,227.2$ \\
\hline Gross domestic absorption & $4,789.6$ & $5,210.4$ & $5,824.8$ & $6,910.6$ & $7,978.0$ & $10,952.9$ & $14,159.4$ \\
\hline Consumption & $3,969.9$ & $4,334.1$ & $4,895.7$ & $5,772.9$ & $6,416.2$ & $8,999.3$ & $10,899.8$ \\
\hline Private & $3,226.6$ & $3,508.0$ & $3,970.0$ & $4,157.5$ & $5,181.9$ & $7,455.7$ & $9,098.6$ \\
\hline Public & 743.3 & 826.1 & 925.6 & $1,615.4$ & $1,234.2$ & $1,543.6$ & $1,801.2$ \\
\hline Gross fixed investment & 819.7 & 876.3 & 929.1 & $1,137.7$ & $1,561.8$ & $1,953.6$ & $3,259.7$ \\
\hline Private & 559.9 & 615.2 & 683.6 & 852.3 & $1,008.9$ & $1,423.7$ & $1,653.8$ \\
\hline Public & 259.9 & 261.0 & 245.6 & 285.4 & 552.9 & 529.9 & $1,605.8$ \\
\hline Change in stocks & .. & .. & .. & .. & .. & .. & .. \\
\hline $\begin{array}{c}\text { Exports of goods and } \\
\text { nonfactor services }\end{array}$ & 2274.22 & 2263.10 & 2416.91 & 2556.27 & 3394.39 & 4739.32 & 6067.76 \\
\hline \multicolumn{8}{|l|}{ Memorandum items: } \\
\hline & \multicolumn{7}{|c|}{ (In percent of GDP) } \\
\hline Consumption & 88.6 & 88.1 & 91.0 & 88.1 & 87.1 & 89.8 & 90.6 \\
\hline Private & 72.0 & 71.3 & 73.8 & 63.4 & 70.4 & 74.4 & 75.6 \\
\hline Public & 16.6 & 16.8 & 17.2 & 24.6 & 16.8 & 15.4 & 15.0 \\
\hline Domestic savings $1 /$ & 11.4 & 11.9 & 9.0 & 11.9 & 12.9 & 10.2 & 9.4 \\
\hline Domestic investment & 18.3 & 17.8 & 17.3 & 17.4 & 21.2 & 19.5 & 27.1 \\
\hline $\begin{array}{c}\text { Imports of goods and } \\
\text { nonfactor services }\end{array}$ & 57.7 & 51.7 & 53.1 & 44.4 & 54.4 & 56.5 & 68.0 \\
\hline $\begin{array}{c}\text { Exports of goods and } \\
\text { nonfactor services }\end{array}$ & 50.8 & 46.0 & 44.9 & 39.0 & 46.1 & 47.3 & 50.4 \\
\hline
\end{tabular}

Sources: Gambian authorities; and IMF staff estimates.

1/ Defined as GDP minus consumption. 
Table 6. The Gambia: Savings-Investment Balance, 1998-2004

\begin{tabular}{|c|c|c|c|c|c|c|c|}
\hline & 1998 & 1999 & 2000 & $\begin{array}{r}2001 \\
\text { Est. }\end{array}$ & $\begin{array}{r}2002 \\
\text { Prel. }\end{array}$ & $\begin{array}{r}2003 \\
\text { Prel. }\end{array}$ & $\begin{array}{r}2004 \\
\text { Prel. }\end{array}$ \\
\hline & \multicolumn{7}{|c|}{ (In percent of GDP) } \\
\hline GDP market prices & 100.0 & 100.0 & 100.0 & 100.0 & 100.0 & 100.0 & 100.0 \\
\hline Factor services, net & -4.8 & -5.0 & -4.9 & -5.5 & -6.0 & -5.4 & -5.0 \\
\hline Remittances of technical assistance staff & 1.4 & 1.9 & 2.4 & 2.1 & 3.0 & 3.5 & 4.7 \\
\hline Interest receipts, net & -6.2 & -6.9 & -7.3 & -7.6 & -9.1 & -8.9 & -9.7 \\
\hline GNP market prices & 95.2 & 95.0 & 95.1 & 94.5 & 94.0 & 94.6 & 95.0 \\
\hline Unrequited transfers, net $1 /$ & 9.3 & 8.0 & 9.9 & 8.3 & 11.6 & 9.6 & 10.9 \\
\hline Of which: official transfers & 8.5 & 7.1 & 7.4 & 7.5 & 10.6 & 8.5 & 9.9 \\
\hline Gross disposable national income & 104.5 & 103.0 & 105.1 & 102.8 & 105.5 & 104.2 & 105.9 \\
\hline Total consumption & 88.6 & 88.1 & 91.0 & 88.1 & 87.1 & 89.8 & 90.6 \\
\hline Private consumption 2/ & 72.0 & 71.3 & 73.8 & 63.4 & 70.4 & 74.4 & 75.6 \\
\hline Government consumption 3/ & 16.6 & 16.8 & 17.2 & 24.6 & 16.8 & 15.4 & 15.0 \\
\hline Gross domestic savings & 11.4 & 11.9 & 9.0 & 11.9 & 12.9 & 10.2 & 9.4 \\
\hline Government domestic savings 4/ & 2.0 & 1.1 & 1.3 & -9.5 & -0.4 & 0.3 & 6.0 \\
\hline Private domestic savings $5 /$ & 9.4 & 10.9 & 7.7 & 21.5 & 13.3 & 9.9 & 3.5 \\
\hline Gross national savings & 15.9 & 14.9 & 14.1 & 14.8 & 18.4 & 14.4 & 15.3 \\
\hline Government national savings & 10.4 & 8.1 & 8.7 & -2.0 & 10.1 & 8.8 & 15.8 \\
\hline Of which: domestically generated & 2.0 & 1.1 & 1.3 & -9.5 & -0.4 & 0.3 & 6.0 \\
\hline Private national savings $4 /$ & 5.5 & 6.8 & 5.4 & 16.8 & 8.2 & 5.6 & -0.5 \\
\hline Total domestically generated 5 / & 7.4 & 7.9 & 6.7 & 7.2 & 7.8 & 5.9 & 5.5 \\
\hline Gross domestic investment & 18.3 & 17.8 & 17.3 & 17.4 & 21.2 & 19.5 & 27.1 \\
\hline Government investment 6/ & 5.8 & 5.3 & 4.6 & 4.4 & 7.5 & 5.3 & 13.3 \\
\hline Private investment $2 /$ & 12.5 & 12.5 & 12.7 & 13.0 & 13.7 & 14.2 & 13.7 \\
\hline Private investment rate (\% of GDP) & 12.5 & 12.5 & 12.7 & 13.0 & 13.7 & 14.2 & 13.7 \\
\hline \multicolumn{8}{|l|}{ Memorandum items: } \\
\hline \multicolumn{8}{|l|}{ External current account } \\
\hline Including transfers & -2.4 & -2.9 & -3.1 & -2.6 & -2.8 & -5.1 & -11.8 \\
\hline Excluding transfers & -10.9 & -9.9 & -10.6 & -10.1 & -13.4 & -13.6 & -21.6 \\
\hline Government financial balance 7/ & -3.8 & -4.2 & -3.3 & -13.9 & -7.9 & -5.0 & -7.4 \\
\hline Government savings & 2.0 & 1.1 & 1.3 & -9.5 & -0.4 & 0.3 & 6.0 \\
\hline Government investment & 5.8 & 5.3 & 4.6 & 4.4 & 7.5 & 5.3 & 13.3 \\
\hline Private financial balance $7 /$ & -7.0 & -5.7 & -7.3 & 3.8 & -5.5 & -8.6 & -14.2 \\
\hline Private savings & 5.5 & 6.8 & 5.4 & 16.8 & 8.2 & 5.6 & -0.5 \\
\hline Private investment & 12.5 & 12.5 & 12.7 & 13.0 & 13.7 & 14.2 & 13.7 \\
\hline
\end{tabular}

Sources: Gambian authorities; and IMF staff estimates.

$1 /$ Consists of both official and private transfers.

2/ Includes public enterprise sector.

3/ Government current expenditure (excluding Gambia Local Fund), less capital component of recurrent budget,

plus current component of development budget.

4/ Domestic revenue (excluding capital revenue) less government consumption.

$5 /$ Gross national savings excluding official transfers.

6/ Development expenditure (excluding net lending), plus capital component of recurrent budget, less current

component of development budget.

7/ Domestically generated financial balances. 
Table 7. The Gambia: Agricultural Production, 1998-2004

\begin{tabular}{|c|c|c|c|c|c|c|c|}
\hline & 1998 & 1999 & 2000 & 2001 & 2002 & 2003 & 2004 \\
\hline Acreage & \multicolumn{7}{|c|}{ (In thousands of hectares) } \\
\hline Export crops & 77.7 & 113.8 & 126.4 & 138.9 & 105.6 & 107.9 & 119.6 \\
\hline Groundnuts & 75.3 & 112.2 & 124.8 & 138.9 & 105.6 & 107.9 & 119.6 \\
\hline Cotton & 2.4 & 1.6 & 1.6 & $\ldots$ & $\ldots$ & $\ldots$ & ... \\
\hline Food crops & 119.5 & 125.3 & 146.3 & 159.0 & 145.6 & 173.4 & 184.7 \\
\hline Rice (paddy) & 18.3 & 15.8 & 16.7 & 18.2 & 12.0 & 17.8 & 20.8 \\
\hline Irrigated 1/ & $\ldots$ & 2.2 & 2.3 & $\ldots$ & $\ldots$ & & \\
\hline Rainfed & 18.3 & 13.5 & 14.4 & $\ldots$ & $\ldots$ & & \\
\hline Sorghum 2/ & 14.2 & 18.5 & 24.4 & 26.2 & 18.3 & 24.7 & 25.6 \\
\hline Millet 2/ & 75.3 & 76.3 & 90.4 & 97.4 & 96.9 & 109.9 & 114.9 \\
\hline Maize & 11.7 & 14.7 & 14.8 & 17.2 & 18.4 & 21.0 & 23.3 \\
\hline Production & \multicolumn{7}{|c|}{ (In thousands of metric tons) } \\
\hline Export crops & 75.0 & 123.4 & 138.2 & 151.7 & 72.0 & 92.9 & 120.5 \\
\hline Groundnuts & 73.5 & 123.0 & 138.0 & 151.1 & 71.5 & 92.9 & 120.5 \\
\hline Cotton & 1.5 & 0.4 & 0.2 & 0.6 & 0.5 & $\ldots$ & $\ldots$ \\
\hline Food crops & 114.1 & 151.1 & 175.6 & 199.9 & 137.0 & 213.3 & 237.5 \\
\hline Rice (paddy) & 26.5 & 31.7 & 34.1 & 32.6 & 18.6 & 29.5 & 40.4 \\
\hline Irrigated 1/ & 7.8 & 13.2 & 13.4 & $\ldots$ & $\ldots$ & $\ldots$ & $\ldots$ \\
\hline Rainfed & 18.7 & 18.3 & 20.7 & $\ldots$ & $\ldots$ & $\ldots$ & $\ldots$ \\
\hline Sorghum 2/ & 9.9 & 18.0 & 24.9 & 33.4 & 15.2 & 30.1 & 32.1 \\
\hline Millet 2/ & 64.7 & 81.0 & 94.6 & 104.9 & 84.6 & 120.3 & 129.0 \\
\hline Maize & 13.0 & 20.4 & 22.0 & 29.0 & 18.6 & 33.4 & 36.1 \\
\hline
\end{tabular}

Source: Gambian authorities.

1/ Including double-cropped area.

2/ Including area intercropped with groundnuts. 
Table 8. The Gambia: Minimum Producer Prices for Agricultural Commodities, 1998-2004 (In dalasis per ton)

\begin{tabular}{lccccccc}
\hline & 1998 & 1999 & 2000 & 2001 & 2002 & 2003 & 2004 \\
\hline Cotton & 3,400 & 3,400 & 3,750 & 3,650 & 4,150 & $\ldots$ & $\ldots$ \\
Groundnuts (undecorticated) & 2,790 & 2,700 & 2,600 & 2,650 & 4,250 & 7,500 & 8,100 \\
Rice (paddy) & 1,750 & 1,750 & 1,750 & 2,250 & 2,500 & $\ldots$ & $\ldots$ \\
\hline
\end{tabular}

Source: Gambian authorities. 
Table 9. The Gambia: Purchases and Disposition of Groundnuts, 1998-2004 (In metric tons)

\begin{tabular}{|c|c|c|c|c|c|c|c|}
\hline & 1998 & 1999 & 2000 & 2001 & 2002 & 2003 & 2004 \\
\hline Delivery purchases (undecorticated) & $\ldots$ & $\ldots$ & 41,912 & 42,813 & 62,203 & 12,833 & 19,145 \\
\hline Export sales & $\ldots$ & $\ldots$ & 20,105 & 25,484 & 40,803 & 7,700 & $\ldots$ \\
\hline Decorticated FAQ groundnuts 1/ & $\ldots$ & $\ldots$ & 15,204 & 8,228 & 2,097 & 3,000 & $\ldots$ \\
\hline Decorticated HPS groundnuts 2/ & $\ldots$ & $\ldots$ & 672 & 7,267 & 3,290 & 4,000 & $\ldots$ \\
\hline Oil & $\ldots$ & $\ldots$ & 3,000 & 1,438 & 15,707 & 700 & $\ldots$ \\
\hline Cake & $\ldots$ & $\ldots$ & 1,229 & 2,581 & 19,709 & 0 & $\ldots$ \\
\hline Local sales 3/ & $\ldots$ & $\ldots$ & 20,700 & 22,400 & $\ldots$ & $\ldots$ & $\ldots$ \\
\hline Seednuts & $\cdots$ & $\cdots$ & $\ldots$ & $\ldots$ & $\ldots$ & $\cdots$ & $\cdots$ \\
\hline Wastage in shelling/processing 4/ & $\ldots$ & $\ldots$ & 13,412 & 13,700 & 21,400 & 5,133 & $\ldots$ \\
\hline
\end{tabular}

Source: Gambian authorities.

$1 / \mathrm{FAQ}=$ fair average quality.

2/ HPS $=$ handpicked selected.

3/ Estimated as 15 percent of total production, including seeds.

4/ Estimated as 32 percent of delivery purchases. 


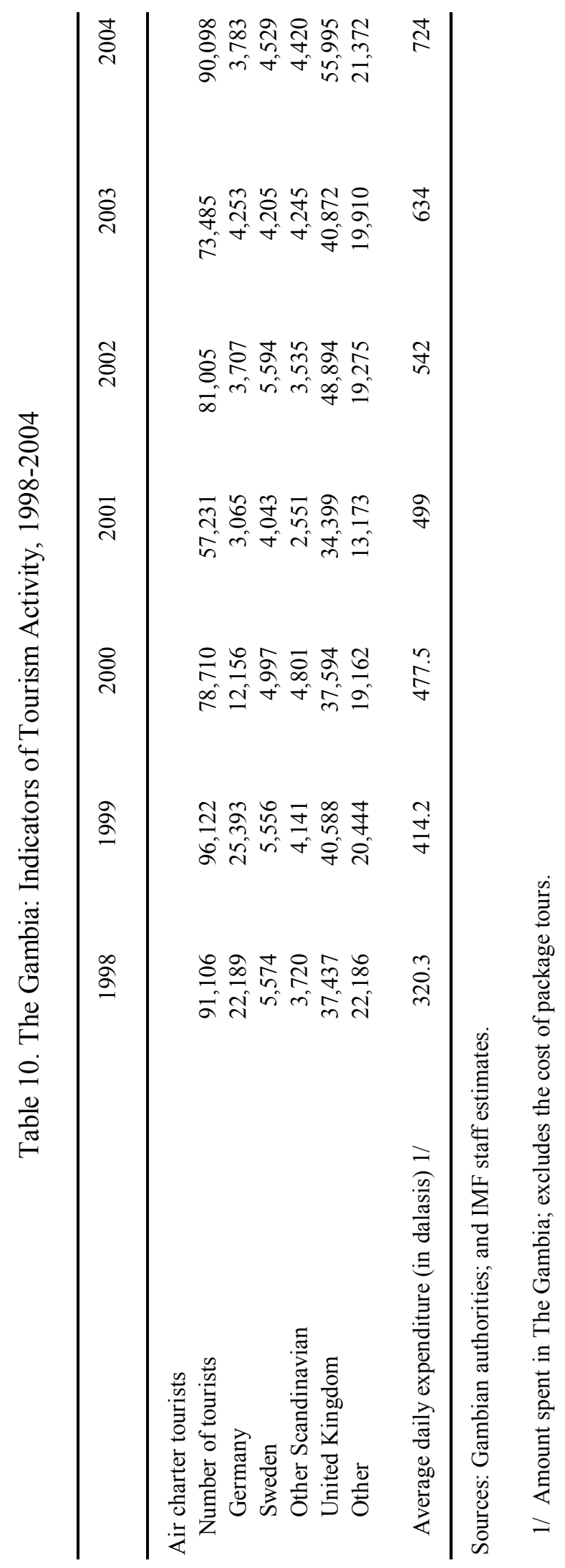


Table 11. The Gambia: Energy Statistics, 1998-2004

\begin{tabular}{|c|c|c|c|c|c|c|c|}
\hline & 1998 & 1999 & 2000 & 2001 & 2002 & 2003 & $\begin{array}{l}2004 \\
\text { Prel }\end{array}$ \\
\hline Electricity & \multicolumn{7}{|c|}{ (In thousands of kilowatt-hours) } \\
\hline Total production & 122,187 & 123,401 & 117,553 & 134,001 & 144,124 & 150,307 & 128,061 \\
\hline Residential consumption & 37,462 & 48,851 & 45,676 & 55,324 & 62,060 & 48,458 & 38,833 \\
\hline $\begin{array}{l}\text { Business consumption } \\
\text { (including government) }\end{array}$ & 33,405 & 23,213 & 21,530 & 25,698 & 24,934 & 44,693 & 41,132 \\
\hline Street lighting & 462 & $\ldots$ & $\ldots$ & 740 & 507 & 383 & 279 \\
\hline Other consumption & 7,274 & 11,337 & 10,347 & 12,917 & 17,301 & $\ldots$ & .. \\
\hline \multicolumn{5}{|l|}{ Losses (including powerhouse } & 39,322 & 56,773 & 47,817 \\
\hline & \multicolumn{7}{|c|}{ (In dalasis per kilowatt-hour) } \\
\hline \multicolumn{8}{|l|}{ Retail electricity prices } \\
\hline Residential & 2.08 & $1.55-2.21$ & $1.55-2.21$ & $\ldots$ & $\ldots$ & $\ldots$ & $1.55-6.98$ \\
\hline Business & 2.21 & 2.21 & 2.21 & $\ldots$ & $\ldots$ & $\ldots$ & 7.25 \\
\hline Hotels/industries & 2.54 & 2.54 & 2.54 & $\ldots$ & $\ldots$ & $\ldots$ & 8.02 \\
\hline & \multicolumn{7}{|c|}{ (In millions of liters) } \\
\hline Petroleum imports & 44.0 & $\ldots$ & $\ldots$ & $\ldots$ & $\ldots$ & $\ldots$ & .. \\
\hline Gas oil & 17.7 & $\ldots$ & $\ldots$ & $\ldots$ & $\ldots$ & $\ldots$ & .. \\
\hline \multirow[t]{2}{*}{ Diesel oil } & 26.4 & $\ldots$ & $\ldots$ & $\ldots$ & $\ldots$ & $\ldots$ & .. \\
\hline & \multicolumn{7}{|c|}{ (In millions of dalasis) } \\
\hline \multirow[t]{2}{*}{ Imports of mineral fuels and products 1 / } & $\ldots$ & 121.3 & 289.4 & 149.1 & 341.7 & 455.4 & .. \\
\hline & \multicolumn{7}{|c|}{ (Dalasis/litre) } \\
\hline \multicolumn{8}{|l|}{ Retail petroleum prices } \\
\hline Gasoline & 8.50 & 8.95 & 8.95 & $\ldots$ & $\ldots$ & 22.00 & 22.00 \\
\hline Diesel oil & 6.50 & 6.50 & 6.75 & $\ldots$ & $\ldots$ & 21.50 & 21.50 \\
\hline
\end{tabular}

Sources: Gambian authorities; and the National Water and Electricity Company Ltd. (NAWEC).

1/ Includes mineral fuels, mineral oils and products of their distillation, bitminous substances, and mineral waxes; c.i.f. value. 


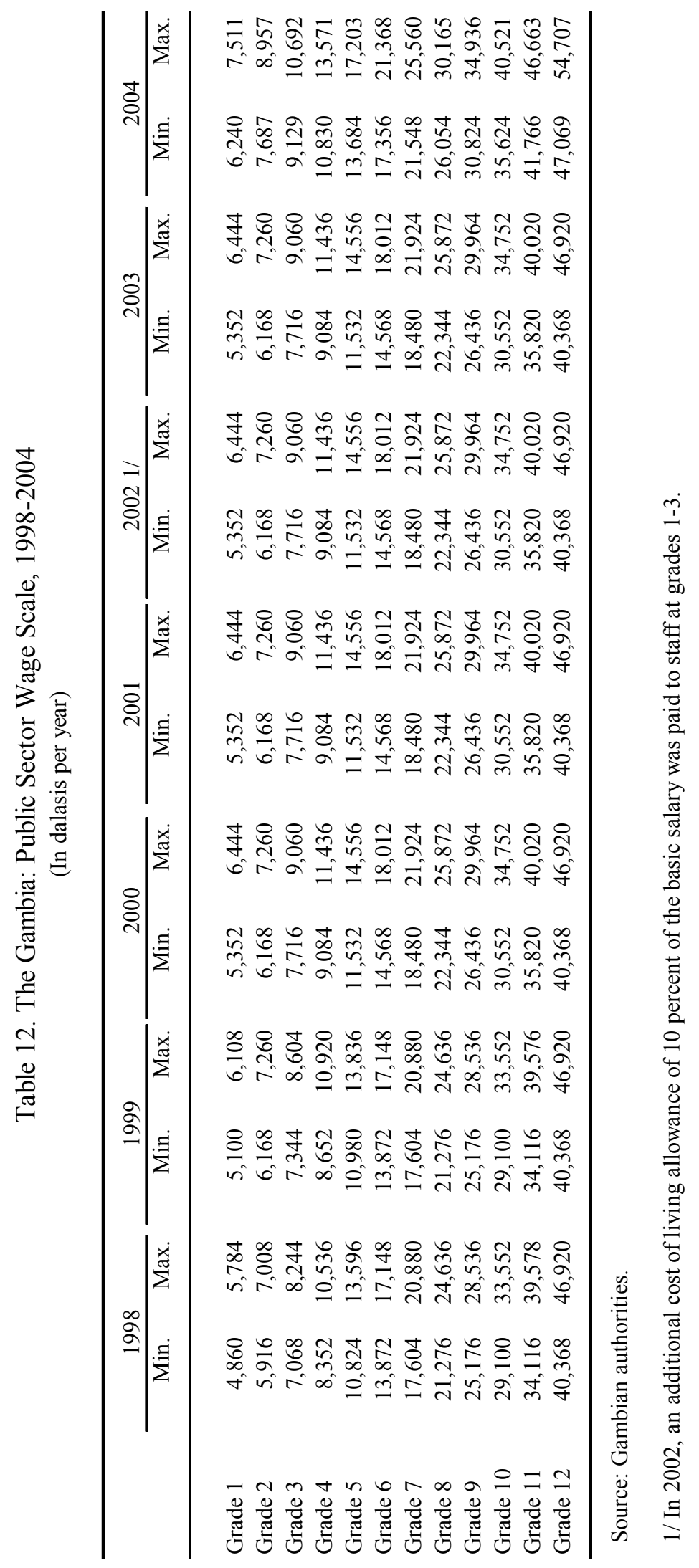




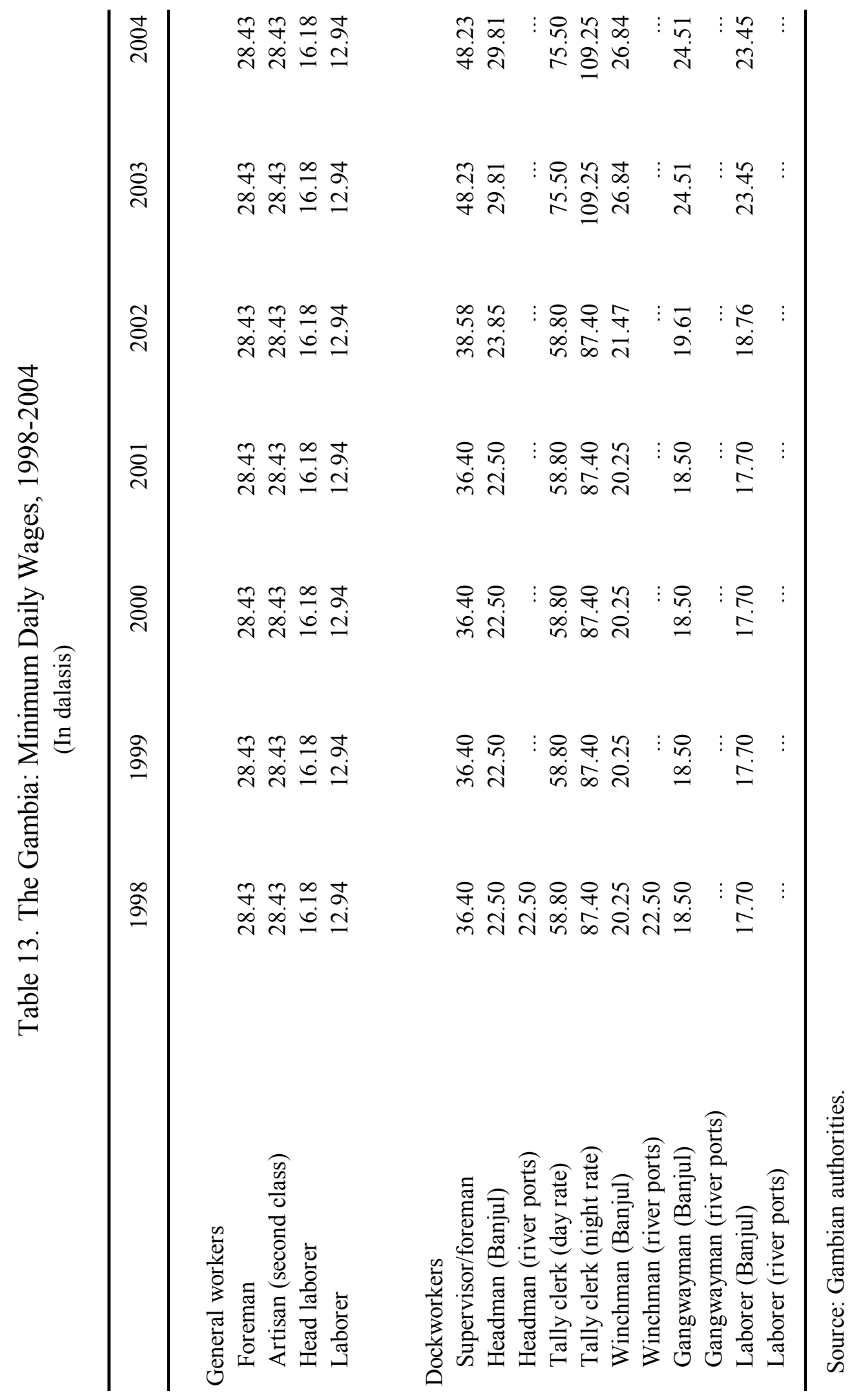


Table 14. The Gambia: Civil Service Structure, 1998-2004 1/

\begin{tabular}{|c|c|c|c|c|c|c|c|}
\hline & 1998 & 1999 & 2000 & 2001 & 2002 & 2003 & 2004 \\
\hline & \multicolumn{7}{|c|}{ (Number of employees) } \\
\hline Executive and legislative offices $2 /$ & 951 & 948 & 1,007 & 814 & 827 & 857 & 838 \\
\hline Defense & 1,456 & 1,573 & 1,708 & $\ldots$ & $\ldots$ & $\ldots$ & 31 \\
\hline Interior & 2,448 & 2,567 & 2,586 & $\ldots$ & $\ldots$ & $\ldots$ & 50 \\
\hline Information and tourism & 51 & 51 & 66 & 66 & 40 & 45 & 45 \\
\hline External affairs & 64 & 68 & 72 & 93 & 104 & 126 & 125 \\
\hline Finance and economic affairs & 470 & 462 & 466 & 584 & 598 & 612 & 620 \\
\hline Local government and lands & 383 & 377 & 381 & 415 & 416 & 413 & 420 \\
\hline Agriculture & 1,146 & 916 & 913 & 920 & 896 & 934 & 963 \\
\hline Natural resources $3 /$ & $\ldots$ & $\ldots$ & $\ldots$ & & $\ldots$ & $\ldots$ & $\ldots$ \\
\hline Works, construction and infrastructure & 619 & 827 & 816 & 346 & 349 & 226 & 228 \\
\hline Trade, industry and employment & 160 & 154 & 155 & 170 & 172 & 146 & 148 \\
\hline Education, youth, and sports & 6,022 & 5,969 & 5,903 & 5,064 & 5,067 & 5,455 & 6,037 \\
\hline \multicolumn{8}{|l|}{ Of which } \\
\hline Gambia Technical Institute & 128 & 129 & $\ldots$ & $\ldots$ & $\ldots$ & $\ldots$ & $\ldots$ \\
\hline Gambia College & 162 & 212 & $\ldots$ & $\ldots$ & $\ldots$ & $\ldots$ & $\ldots$ \\
\hline National Council of Arts and Culture & 51 & 52 & $\ldots$ & $\ldots$ & $\ldots$ & $\ldots$ & $\ldots$ \\
\hline Health & 1,474 & 1,517 & 1,586 & 1,859 & 1,878 & 1,569 & 1,549 \\
\hline \multicolumn{8}{|l|}{ Of which } \\
\hline Royal Victoria Hospital & 874 & 866 & 887 & 902 & 938 & 936 & $\ldots$ \\
\hline Bansang Hospital & 264 & 274 & 272 & 307 & 305 & 305 & $\ldots$ \\
\hline Autonomous organizations 4/ & 272 & 278 & 281 & 283 & 299 & 296 & $\ldots$ \\
\hline \multicolumn{8}{|l|}{ Of which } \\
\hline Agricultural Research (NARI) & 134 & 137 & 137 & 137 & 148 & 148 & $\ldots$ \\
\hline Environmental Agency & 36 & 39 & 42 & 42 & 47 & 47 & $\ldots$ \\
\hline \multirow[t]{2}{*}{ Total } & 15,516 & 15,707 & 15,940 & $\ldots$ & $\ldots$ & $\ldots$ & $\ldots$ \\
\hline & \multicolumn{7}{|c|}{ (In percent) } \\
\hline Executive and legislative offices $2 /$ & 6.1 & 6.0 & 6.3 & $\ldots$ & $\ldots$ & $\ldots$ & $\ldots$ \\
\hline Defense & 9.4 & 10.0 & 10.7 & $\ldots$ & $\ldots$ & $\ldots$ & $\ldots$ \\
\hline Interior & 15.8 & 16.3 & 16.2 & $\ldots$ & $\ldots$ & $\ldots$ & $\ldots$ \\
\hline Information and tourism & 0.3 & 0.3 & 0.4 & $\ldots$ & $\ldots$ & $\ldots$ & $\ldots$ \\
\hline External affairs & 0.4 & 0.4 & 0.5 & $\ldots$ & $\ldots$ & $\ldots$ & $\ldots$ \\
\hline Finance and economic affairs & 3.0 & 2.9 & 2.9 & $\ldots$ & $\ldots$ & $\ldots$ & $\ldots$ \\
\hline Local government and lands & 2.5 & 2.4 & 2.4 & $\ldots$ & $\ldots$ & $\ldots$ & $\ldots$ \\
\hline Agriculture & 7.4 & 5.8 & 5.7 & $\ldots$ & $\ldots$ & $\ldots$ & $\ldots$ \\
\hline Natural resources $3 /$ & $\ldots$ & $\ldots$ & $\ldots$ & & $\ldots$ & $\ldots$ & $\ldots$ \\
\hline Works and communications & 4.0 & 5.3 & 5.1 & $\ldots$ & $\ldots$ & $\ldots$ & $\ldots$ \\
\hline Trade and industry & 1.0 & 1.0 & 1.0 & $\ldots$ & $\ldots$ & $\ldots$ & $\ldots$ \\
\hline Education, youth, and sports & 38.8 & 38.0 & 37.0 & $\ldots$ & $\ldots$ & $\ldots$ & $\ldots$ \\
\hline Health & 9.5 & 9.7 & 9.9 & $\ldots$ & $\ldots$ & $\ldots$ & $\ldots$ \\
\hline Autonomous organizations 4/ & 1.8 & 1.8 & 1.8 & $\ldots$ & $\ldots$ & $\ldots$ & $\ldots$ \\
\hline Total & 100.0 & 100.0 & 100.0 & $\ldots$ & $\ldots$ & $\ldots$ & $\ldots$ \\
\hline
\end{tabular}

Source: Gambian authorities.

1/ From 2000 onward, data are based on budget figures.

2/ Includes the Office of the President, legislature, judiciary, Public Service Commission, and National Audit Office. 


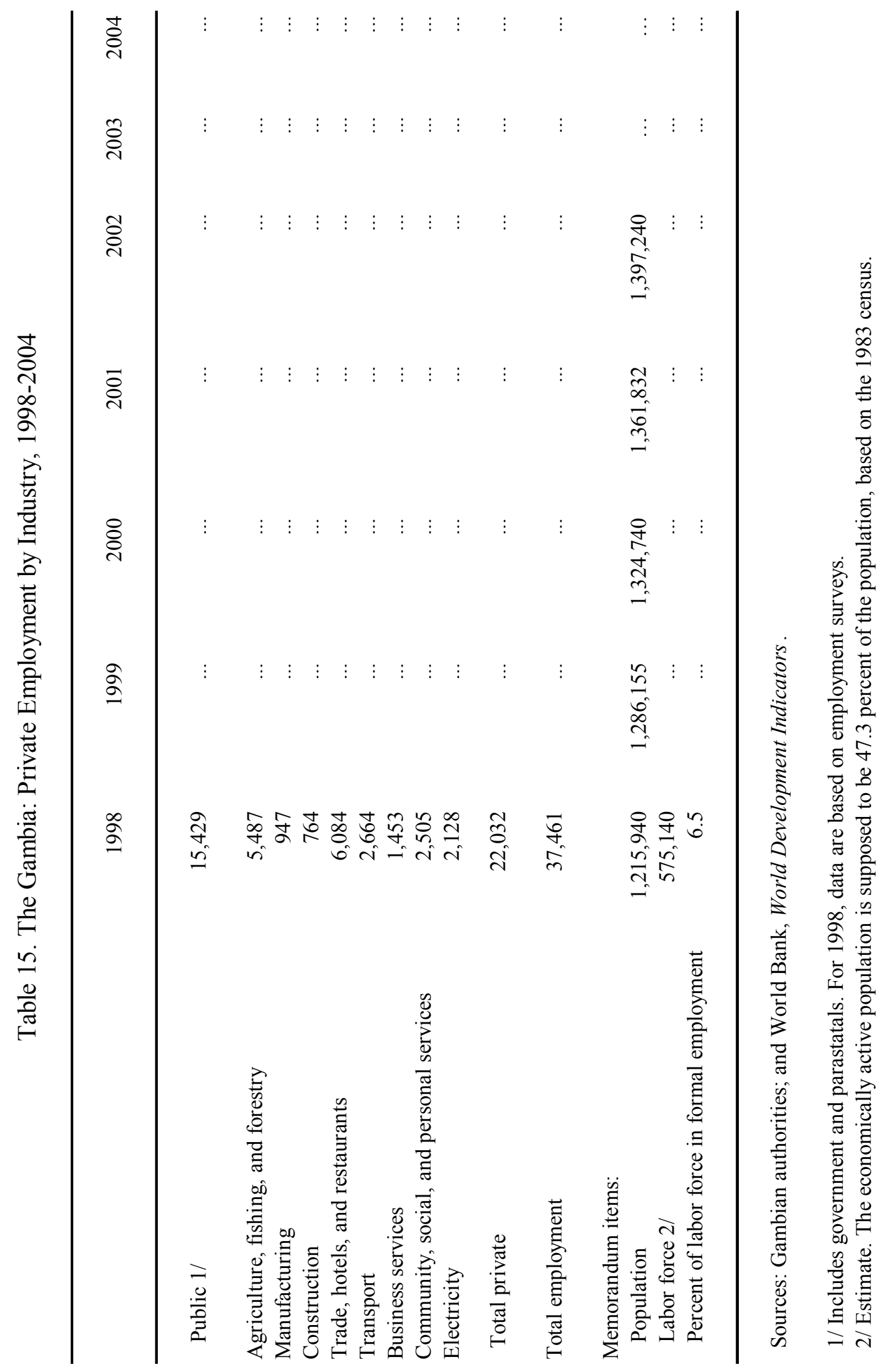




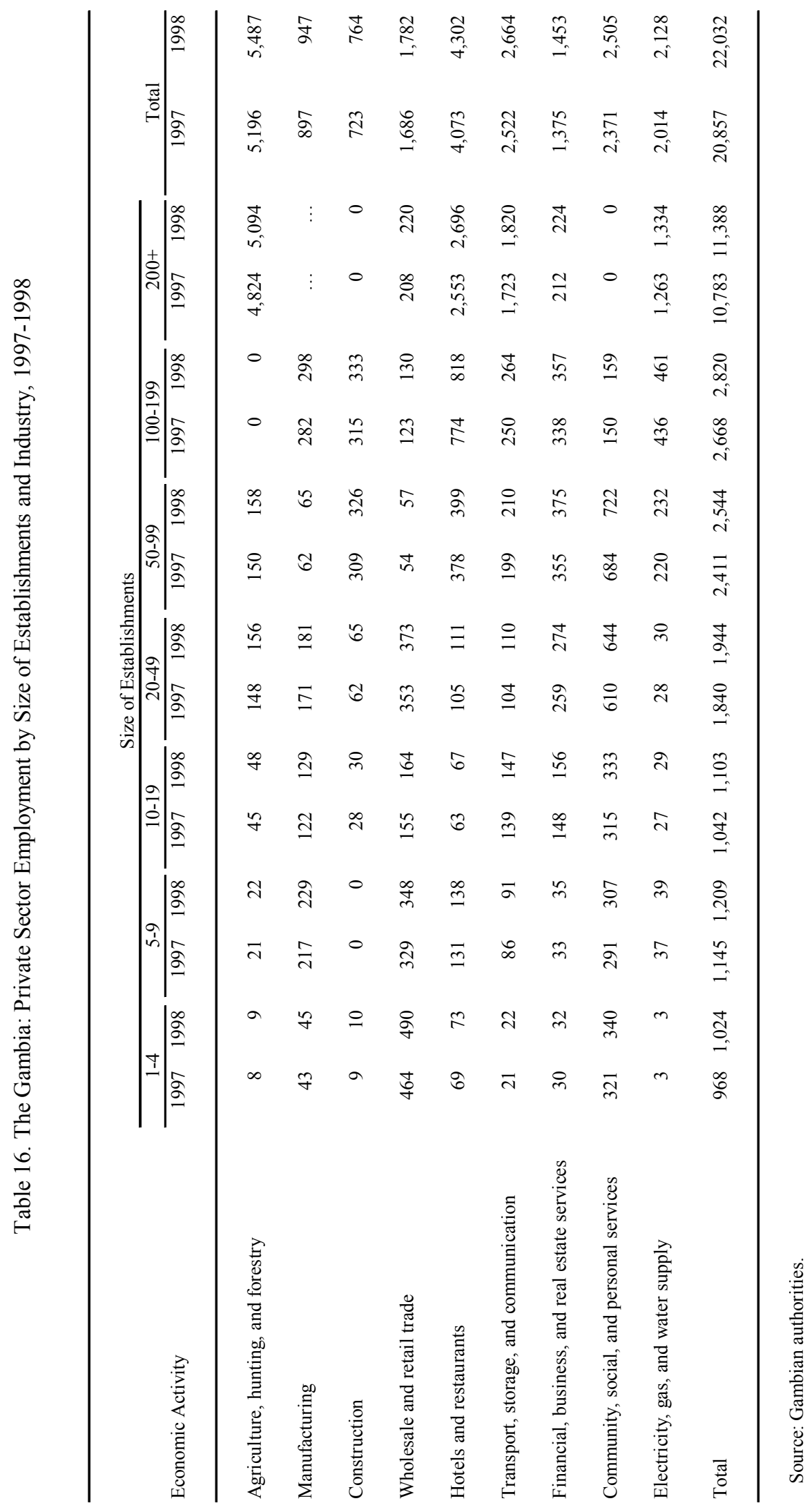


Table 17. The Gambia: Overall Consumer Price Index for Low-Income Households in Banjul and Kombo St. Mary, January 1998-March 2005

(Base year, 1974=100, unless otherwise indicated)

\begin{tabular}{lrrrrrrrr}
\hline & 1998 & 1999 & 2000 & 2001 & 2002 & 2003 & 2004 & 2005 \\
& & & & & & & & \\
& & & & & & & & \\
January & $1,379.7$ & $1,501.3$ & $1,523.9$ & $1,576.7$ & $1,682.6$ & $1,876.4$ & $2,216.0$ & 2369.3 \\
February & $1,407.0$ & $1,501.8$ & $1,524.0$ & $1,577.1$ & $1,681.7$ & $1,904.9$ & $2,243.0$ & 2370.6 \\
March & $1,407.2$ & $1,502.6$ & $1,524.0$ & $1,577.3$ & $1,682.3$ & $1,929.7$ & $2,260.3$ & 2372.6 \\
April & $1,420.5$ & $1,502.7$ & $1,525.3$ & $1,577.4$ & $1,684.3$ & $1,971.7$ & $2,282.8$ & $\ldots$ \\
May & $1,413.9$ & $1,502.5$ & $1,525.4$ & $1,578.3$ & $1,685.0$ & $1,983.9$ & $2,296.9$ & $\ldots$ \\
June & $1,490.3$ & $1,502.6$ & $1,525.6$ & $1,586.7$ & $1,685.6$ & $2,006.2$ & $2,322.8$ & $\ldots$ \\
July & $1,477.3$ & $1,522.5$ & $1,526.4$ & $1,592.6$ & $1,686.7$ & $2,027.0$ & $2,344.7$ & $\ldots$ \\
August & $1,495.2$ & $1,523.1$ & $1,527.1$ & $1,599.4$ & $1,697.0$ & $2,054.6$ & $2,356.3$ & $\ldots$ \\
September & $1,497.5$ & $1,523.8$ & $1,526.8$ & $1,603.2$ & $1,779.5$ & $2,098.9$ & $2,357.5$ & $\ldots$ \\
October & $1,499.4$ & $1,523.0$ & $1,526.0$ & $1,605.3$ & $1,807.9$ & $2,119.2$ & $2,360.0$ & $\ldots$ \\
November & $1,502.2$ & $1,523.8$ & $1,526.5$ & $1,606.3$ & $1,840.1$ & $2,151.6$ & $2,363.2$ & $\ldots$ \\
December & $1,497.6$ & $1,523.8$ & $1,526.9$ & $1,650.1$ & $1,864.7$ & $2,192.2$ & $2,367.4$ & $\ldots$
\end{tabular}

Memorandum items:

$\begin{aligned} & \text { Overall index } \\ & \text { (period average) }\end{aligned}$
$\begin{aligned} & \text { Annual percentage change 1/ } \\ & \quad \text { (period average) }\end{aligned}$
$\begin{aligned} & \text { Annual percentage change 2/ } \\ & \quad \text { (end of period) }\end{aligned}$

Source: Gambian authorities.

1/ Based on average for Q1, 2004 and Q1, 2005.

2/ Based on data for end-March 2005. 
Table 18. The Gambia: Central Government Operations, 1998-04 (In millions of dalasis)

\begin{tabular}{|c|c|c|c|c|c|c|c|}
\hline & 1998 & 1999 & 2000 & 2001 & 2002 & 2003 & 2004 \\
\hline Revenue and grants & 919.9 & 944.5 & $1,117.2$ & $1,125.7$ & $1,528.7$ & $1,820.4$ & $3,065.0$ \\
\hline Domestic revenue & 831.5 & 878.7 & 995.4 & 989.9 & $1,201.8$ & $1,574.2$ & $2,517.8$ \\
\hline Tax revenue & 751.1 & 773.7 & 869.9 & 853.8 & $1,040.2$ & $1,380.7$ & $2,244.7$ \\
\hline Direct tax & 185.1 & 201.8 & 223.6 & 251.0 & 318.0 & 441.0 & 606.3 \\
\hline Of which: personal & 76.4 & 81.3 & 90.4 & 102.6 & 122.4 & 154.2 & 207.2 \\
\hline corporate & 93.7 & 102.2 & 115.0 & 132.7 & 176.8 & 265.8 & 367.1 \\
\hline Indirect tax & 566.0 & 571.8 & 646.3 & 602.8 & 722.2 & 939.7 & $1,638.4$ \\
\hline Domestic tax on goods and services & 65.3 & 77.3 & 72.8 & 73.9 & 124.9 & 205.7 & 291.4 \\
\hline Tax on international trade & 500.7 & 494.5 & 573.5 & 528.9 & 597.3 & 734.0 & $1,347.0$ \\
\hline Nontax revenue & 80.4 & 105.0 & 125.4 & 136.0 & 161.5 & 193.5 & 273.1 \\
\hline Grants & 88.5 & 65.8 & 121.8 & 135.9 & 326.9 & 246.2 & 547.2 \\
\hline Program & 25.6 & 11.3 & 60.3 & 0.0 & 94.9 & 0.0 & 0.0 \\
\hline Projects & 62.9 & 54.5 & 61.5 & 67.9 & 109.9 & 132.0 & 395.7 \\
\hline HIPC & 0.0 & 0.0 & 0.0 & 68.0 & 122.1 & 114.2 & 151.4 \\
\hline Expenditure and net lending 1/ & 1028.4 & 1118.2 & $1,192.1$ & $2,037.4$ & $1,870.7$ & $2,292.7$ & $3,750.4$ \\
\hline Current expenditure & 799.8 & 887.0 & 985.8 & $1,237.1$ & $1,318.2$ & $1,707.0$ & $2,035.8$ \\
\hline Wages and salaries & 282.9 & 301.7 & 341.2 & 342.0 & 395.2 & 452.6 & 517.5 \\
\hline Other charges & 279.9 & 336.9 & 397.4 & 533.4 & 512.5 & 594.5 & 583.8 \\
\hline Interest & 236.9 & 248.3 & 247.3 & 293.8 & 370.5 & 607.6 & 867.9 \\
\hline External & 56.4 & 60.9 & 60.2 & 68.7 & 84.0 & 163.4 & 234.6 \\
\hline Domestic & 180.4 & 187.5 & 187.1 & 225.0 & 286.6 & 444.2 & 633.3 \\
\hline HIPC Initiative funded expenditure & & & $\ldots$ & 68.0 & 39.9 & 52.3 & 66.6 \\
\hline Capital expenditure and net lending 1 / & 228.7 & 231.2 & 206.3 & 800.3 & 552.5 & 585.7 & $1,714.6$ \\
\hline Capital expenditure & 259.9 & 261.0 & 245.6 & 732.5 & 585.3 & 608.3 & $1,733.5$ \\
\hline External & 211.2 & 221.0 & 196.7 & 224.5 & 495.2 & 472.7 & $1,517.0$ \\
\hline Loans & 148.3 & 155.2 & 135.2 & 156.6 & 368.4 & 340.7 & $1,121.3$ \\
\hline Grants & 62.9 & 65.8 & 61.5 & 67.9 & 126.8 & 132.0 & 395.7 \\
\hline Gambia Local Fund & 48.7 & 40.1 & 48.9 & 60.9 & 57.7 & 57.2 & 88.8 \\
\hline HIPC Initiative funded expenditure & & & $\ldots$ & $\ldots$ & 32.4 & 78.4 & 127.7 \\
\hline Extrabudgetary expenditure 1/ & 0.0 & 0.0 & 0.0 & 447.1 & 0.0 & 0.0 & 4.5 \\
\hline Net lending & -31.2 & -29.8 & -39.3 & 67.8 & -32.8 & -22.6 & -23.4 \\
\hline Overall balance (commitment basis), including grants & -108.5 & -173.7 & -75.0 & -911.6 & -342.0 & -472.3 & -685.5 \\
\hline Excluding grants & -197.0 & -239.5 & -196.8 & $-1,047.5$ & -668.9 & -718.5 & $-1,232.6$ \\
\hline Adjustment to cash basis (float) & 6.1 & -34.5 & -23.7 & -34.7 & 17.8 & -123.6 & -62.6 \\
\hline \multicolumn{8}{|l|}{ Overall balance (cash basis) } \\
\hline Including grants & -133.0 & -208.2 & -98.7 & -946.3 & -324.2 & -595.9 & -748.0 \\
\hline Excluding grants & -221.4 & -274.0 & -220.5 & -1082.2 & -651.1 & -842.1 & -1295.2 \\
\hline Financing & 133.0 & 208.2 & 98.7 & 946.3 & 324.2 & 596.0 & 748.0 \\
\hline External (net) & 54.5 & 30.0 & -45.6 & -23.6 & 140.9 & 59.7 & 690.3 \\
\hline Borrowing & 148.3 & 155.2 & 135.2 & 233.6 & 725.8 & 340.7 & $1,121.3$ \\
\hline Project & 148.3 & 155.2 & 135.2 & 156.6 & 368.4 & 340.7 & $1,121.3$ \\
\hline Program & 0.0 & 0.0 & 0.0 & 0.0 & 0.0 & 0.0 & 0.0 \\
\hline Other loans $2 /$ & 0.0 & 0.0 & 0.0 & 77.0 & 357.4 & 0.0 & 0.0 \\
\hline Amortization & -93.8 & -125.2 & -180.8 & -257.2 & -591.9 & -293.6 & -431.0 \\
\hline HIPC Initiative debt relief & $\ldots$ & $\ldots$ & $\ldots$ & 0.0 & 7.0 & 12.6 & 0.0 \\
\hline Change in arrears (+ increase) & $\ldots$ & $\ldots$ & $\ldots$ & $\ldots$ & $\ldots$ & $\ldots$ & $\ldots$ \\
\hline Domestic & 78.5 & 178.2 & 144.3 & 969.9 & 183.3 & 536.3 & 57.7 \\
\hline Bank 1/ & -20.0 & 71.2 & 45.0 & 952.8 & 22.9 & 401.2 & -578.0 \\
\hline Nonbank & 86.5 & 93.2 & 139.5 & 68.1 & 197.0 & 95.1 & 635.7 \\
\hline Accumulation / repayment (minus) of arrears & 12.0 & 13.9 & -40.2 & -51.0 & -36.7 & 0.0 & 0.0 \\
\hline Privatization proceeds & $\ldots$ & $\ldots$ & 0.0 & 0.0 & 0.0 & 0.0 & 0.0 \\
\hline Repayment of domestic debt & & & 0.0 & 0.0 & 0.0 & 0.0 & 0.0 \\
\hline CBG (unrealized profits) & $\ldots$ & $\ldots$ & $\ldots$ & $\ldots$ & $\ldots$ & 40.0 & 0.0 \\
\hline Financing gap & 0.0 & 0.0 & 0.0 & 0.0 & 0.0 & 0.0 & 0.0 \\
\hline Basic primary balance 3 / & 251.1 & 229.8 & 247.2 & -529.2 & 196.8 & 361.8 & $1,152.3$ \\
\hline \multicolumn{8}{|l|}{ Memorandum items: } \\
\hline Stock of domestic debt & 1146.8 & 1328.6 & $1,693.5$ & $2,496.1$ & $2,694.2$ & $2,513.5$ & $3,697.4$ \\
\hline
\end{tabular}

Sources: Gambian authorities; and IMF staff estimates and projections.

1/ Data for 2001 include US\$28.5 million capital expenditure financed from a retroactive loan from the Central Bank of The Gambia (CBG), which the authorities indicat in October 2003 had not been recorded in official accounts.

2/ Includes loan disbursements (D 287.8 million in January and D 94.7 million in April) from Taiwan Province of China for electricity generators and improvement of distribution network. The generators were delivered to the National Water and Electricity Corporation in October 2001. The inflows were used to pay the suppliers.

3/ Domestic revenue minus total expenditure and net lending, excluding interest payments and externally financed capital expenditure. 
Table 19. The Gambia: Central Government Operations, 1998-2004 (In percent of GDP, unless otherwise indicated)

\begin{tabular}{|c|c|c|c|c|c|c|c|}
\hline & 1998 & 1999 & 2000 & 2001 & 2002 & 2003 & 2004 \\
\hline Revenue and grants & 20.5 & 19.2 & 20.8 & 17.2 & 20.8 & 18.2 & 25.5 \\
\hline Domestic revenue & 18.6 & 17.9 & 18.5 & 15.1 & 16.3 & 15.7 & 20.9 \\
\hline Tax revenue & 16.8 & 15.7 & 16.2 & 13.0 & 14.1 & 13.8 & 18.6 \\
\hline Direct tax & 4.1 & 4.1 & 4.2 & 3.8 & 4.3 & 4.4 & 5.0 \\
\hline Of which: personal & 1.7 & 1.7 & 1.7 & 1.6 & 1.7 & 1.5 & 1.7 \\
\hline corporate & 2.1 & 2.1 & 2.1 & 2.0 & 2.4 & 2.7 & 3.0 \\
\hline Indirect tax & 12.6 & 11.6 & 12.0 & 9.2 & 9.8 & 9.4 & 13.6 \\
\hline Domestic tax on goods and services & 1.5 & 1.6 & 1.4 & 1.1 & 1.7 & 2.1 & 2.4 \\
\hline Tax on international trade & 11.2 & 10.0 & 10.7 & 8.1 & 8.1 & 7.3 & 11.2 \\
\hline Nontax revenue & 1.8 & 2.1 & 2.3 & 2.1 & 2.2 & 1.9 & 2.3 \\
\hline Grants & 2.0 & 1.3 & 2.3 & 2.1 & 4.4 & 2.5 & 4.5 \\
\hline Program & 0.6 & 0.2 & 1.1 & 0.0 & 1.3 & 0.0 & 0.0 \\
\hline Projects & 1.4 & 1.1 & 1.1 & 1.0 & 1.5 & 1.3 & 3.3 \\
\hline HIPC Initiative & 0.0 & 0.0 & 0.0 & 1.0 & 1.7 & 1.1 & 1.3 \\
\hline Expenditure and net lending 1/ & 23.0 & 22.7 & 22.1 & 31.1 & 25.4 & 22.9 & 31.2 \\
\hline Current expenditure & 17.9 & 18.0 & 18.3 & 18.9 & 17.9 & 17.0 & 16.9 \\
\hline Wages and salaries & 6.3 & 6.1 & 6.3 & 5.2 & 5.4 & 4.5 & 4.3 \\
\hline Other charges & 6.2 & 6.8 & 7.4 & 8.1 & 7.0 & 5.9 & 4.9 \\
\hline Interest & 5.3 & 5.0 & 4.6 & 4.5 & 5.0 & 6.1 & 7.2 \\
\hline External & 1.3 & 1.2 & 1.1 & 1.0 & 1.1 & 1.6 & 1.9 \\
\hline Domestic & 4.0 & 3.8 & 3.5 & 3.4 & 3.9 & 4.4 & 5.3 \\
\hline HIPC Initiative funded expenditure & 0.0 & 0.0 & 0.0 & 1.0 & 0.5 & 0.5 & 0.6 \\
\hline Capital expenditure and net lending 1 / & 5.1 & 4.7 & 3.8 & 12.2 & 7.5 & 5.8 & 14.2 \\
\hline Capital expenditure & 5.8 & 5.3 & 4.6 & 11.2 & 7.9 & 6.1 & 14.4 \\
\hline External & 4.7 & 4.5 & 3.7 & 3.4 & 6.7 & 4.7 & 12.6 \\
\hline Loans & 3.3 & 3.2 & 2.5 & 2.4 & 5.0 & 3.4 & 9.3 \\
\hline Grants & 1.4 & 1.3 & 1.1 & 1.0 & 1.7 & 1.3 & 3.3 \\
\hline Gambia Local Fund & 1.1 & 0.8 & 0.9 & 0.9 & 0.8 & 0.6 & 0.7 \\
\hline HIPC Initiative funded expenditure & $\cdots$ & $\cdots$ & $\cdots$ & $\cdots$ & 0.4 & 0.8 & 1.1 \\
\hline Extrabudgetary expenditure 1/ & 0.0 & 0.0 & 0.0 & 6.8 & 0.0 & 0.0 & 0.0 \\
\hline Net lending & -0.7 & -0.6 & -0.7 & 1.0 & -0.4 & -0.2 & -0.2 \\
\hline Overall balance (commitment basis), including grants & -2.4 & -3.5 & -1.4 & -13.9 & -4.6 & -4.7 & -5.7 \\
\hline Excluding grants & -4.4 & -4.9 & -3.7 & -16.0 & -9.1 & -7.2 & -10.2 \\
\hline Adjustment to cash basis (float) & 0.1 & -0.7 & -0.4 & -0.5 & 0.2 & -1.2 & -0.5 \\
\hline Overall balance (cash basis), including grants & -3.0 & -4.2 & -1.8 & -14.4 & -4.4 & -5.9 & -6.2 \\
\hline Excluding grants & -4.9 & -5.6 & -4.1 & -16.5 & -8.8 & -8.4 & -10.8 \\
\hline Financing & 3.0 & 4.2 & 1.8 & 14.4 & 4.4 & 5.9 & 6.2 \\
\hline External (net) & 1.2 & 0.6 & -0.8 & -0.4 & 1.9 & 0.6 & 5.7 \\
\hline Borrowing & 3.3 & 3.2 & 2.5 & 3.6 & 9.9 & 3.4 & 9.3 \\
\hline Project & 3.3 & 3.2 & 2.5 & 2.4 & 5.0 & 3.4 & 9.3 \\
\hline Program & 0.0 & 0.0 & 0.0 & 0.0 & 0.0 & 0.0 & 0.0 \\
\hline Other loans 2/ & 0.0 & 0.0 & 0.0 & 1.2 & 4.9 & 0.0 & 0.0 \\
\hline Amortization & -2.1 & -2.5 & -3.4 & -3.9 & -8.0 & -2.9 & -3.6 \\
\hline HIPC Initiative debt relief & 0.0 & 0.0 & 0.0 & 0.0 & 0.1 & 0.1 & 0.0 \\
\hline Change in arrears (+ increase) & $\cdots$ & $\cdots$ & $\cdots$ & $\cdots$ & $\cdots$ & $\cdots$ & $\cdots$ \\
\hline Domestic & 1.8 & 3.6 & 2.7 & 14.8 & 2.5 & 5.3 & 0.5 \\
\hline Bank 1/ & -0.4 & 1.4 & 0.8 & 14.5 & 0.3 & 4.0 & -4.8 \\
\hline Nonbank & 1.9 & 1.9 & 2.6 & 1.0 & 2.7 & 0.9 & 5.3 \\
\hline Accumulation / repayment (minus) of arrears & 0.3 & 0.3 & -0.7 & -0.8 & -0.5 & 0.0 & 0.0 \\
\hline Privatization proceeds & 0.0 & 0.0 & 0.0 & 0.0 & 0.0 & 0.0 & 0.0 \\
\hline Repayment of domestic debt & 0.0 & 0.0 & 0.0 & 0.0 & 0.0 & 0.0 & 0.0 \\
\hline CBG (unrealized profits) & 0.0 & 0.0 & 0.0 & 0.0 & 0.0 & 0.4 & 0.0 \\
\hline Financing gap & 0.0 & 0.0 & 0.0 & 0.0 & 0.0 & 0.0 & 0.0 \\
\hline Basic primary balance 3 / & 5.6 & 4.7 & 4.6 & -8.1 & 2.7 & 3.6 & 9.6 \\
\hline \multicolumn{8}{|l|}{ Memorandum item: } \\
\hline Stock of domestic debt & 25.6 & 27.0 & 31.5 & 38.1 & 36.6 & 25.1 & 30.7 \\
\hline
\end{tabular}




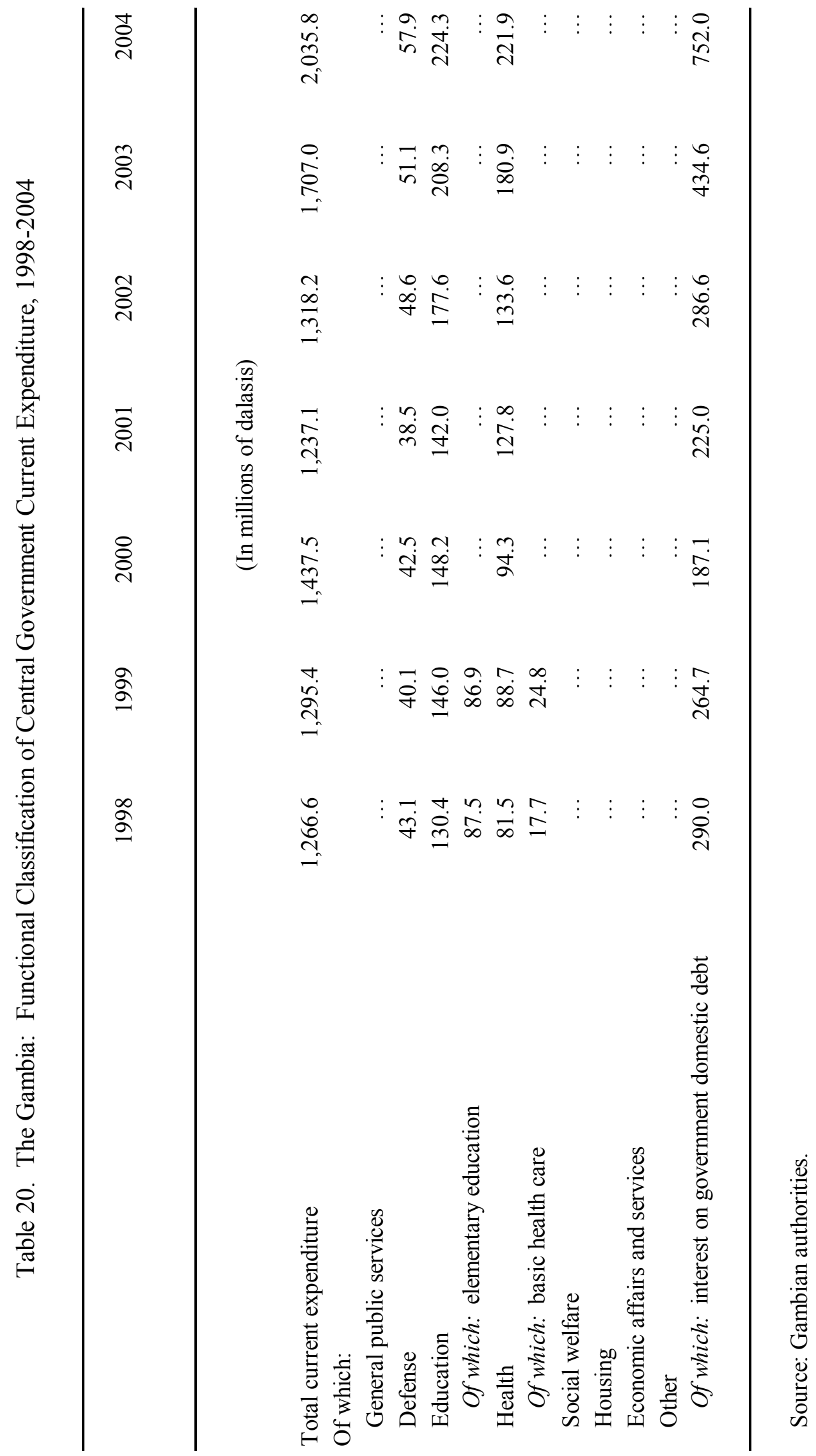


Table 21. The Gambia: Central Government Social Expenditure and Social Indicators, 1998-2004

\begin{tabular}{|c|c|c|c|c|c|c|c|}
\hline & 1998 & 1999 & 2000 & 2001 & 2002 & 2003 & 2004 \\
\hline & \multicolumn{7}{|c|}{ (In millions of dalasis) } \\
\hline Education expenditure & 154.5 & 273.0 & 331.6 & 297.8 & 354.6 & 112.0 & 293.5 \\
\hline \multicolumn{8}{|l|}{ Of which } \\
\hline Primary education & 63.3 & 111.9 & 136.0 & $\ldots$ & $\ldots$ & $\ldots$ & 153.5 \\
\hline Secondary education & 34.0 & 60.1 & 73.0 & $\ldots$ & $\ldots$ & $\ldots$ & $\ldots$ \\
\hline Higher education & 27.8 & 49.1 & 59.7 & $\ldots$ & $\ldots$ & $\ldots$ & $\ldots$ \\
\hline Health expenditure & 83.0 & 175.0 & 253.9 & 192.8 & 225.3 & 146.7 & 164.5 \\
\hline Primary and secondary health care & 36.7 & 77.4 & 112.2 & $\ldots$ & $\ldots$ & 79.7 & 116.1 \\
\hline Tertiary health care & 34.1 & 71.9 & 104.4 & $\ldots$ & $\ldots$ & 66.8 & 48.1 \\
\hline \multirow[t]{2}{*}{ Administration and training } & 12.2 & 25.7 & 37.3 & $\ldots$ & $\ldots$ & 2.0 & 3.0 \\
\hline & \multicolumn{7}{|c|}{ (In percent, unless otherwise indicated) } \\
\hline \multicolumn{8}{|l|}{ Social indicators } \\
\hline Illiteracy rate & 65.7 & 64.5 & 63.4 & 62.2 & 61.1 & $\ldots$ & $\ldots$ \\
\hline \multicolumn{8}{|l|}{ Gross school enrollment rate } \\
\hline Primary school enrollment & $\ldots$ & 69.8 & $\ldots$ & 75.0 & 91.0 & $\ldots$ & $\ldots$ \\
\hline Of which: female & $\ldots$ & $\ldots$ & $\ldots$ & $\ldots$ & $\ldots$ & $\ldots$ & $\ldots$ \\
\hline Secondary school enrollment & $\ldots$ & $\ldots$ & $\ldots$ & $\ldots$ & $\ldots$ & $\ldots$ & $\ldots$ \\
\hline Of which: female & $\ldots$ & $\ldots$ & $\ldots$ & $\ldots$ & $\cdots$ & $\ldots$ & $\ldots$ \\
\hline Life expectancy at birth (years) & 53.2 & 53.2 & $\ldots$ & 53.7 & $\ldots$ & $\ldots$ & $\ldots$ \\
\hline Population with access to health care & $\ldots$ & $\ldots$ & $\ldots$ & $\ldots$ & $\ldots$ & $\ldots$ & $\ldots$ \\
\hline Population with access to safe water & $\ldots$ & $\ldots$ & 62.0 & $\ldots$ & $\ldots$ & $\ldots$ & $\ldots$ \\
\hline Population below the poverty line & $\ldots$ & $\ldots$ & $\ldots$ & $\ldots$ & $\ldots$ & $\ldots$ & $\ldots$ \\
\hline \multicolumn{8}{|l|}{ Infant mortality rate } \\
\hline (per 1,000 live births) & 76.4 & 74.8 & $\ldots$ & 91.0 & $\ldots$ & $\ldots$ & $\ldots$ \\
\hline
\end{tabular}


Table 22. The Gambia: Public Investment Program and Its Financing, 1998-2004

\begin{tabular}{|c|c|c|c|c|c|c|c|}
\hline & 1998 & 1999 & 2000 & 2001 & 2002 & 2003 & 2004 \\
\hline & \multicolumn{7}{|c|}{ (In millions of dalasis) } \\
\hline Agricultural/natural resources & 55.8 & 68.4 & 134.3 & 100.0 & 154.2 & 114.3 & 320.0 \\
\hline Jahally/Pacharr rice project & 1.9 & $\ldots$ & $\ldots$ & $\ldots$ & $\ldots$ & & $\ldots$ \\
\hline Rice development project & 4.8 & $\ldots$ & $\ldots$ & $\ldots$ & 11.0 & 9.0 & 28.7 \\
\hline Cotton development project & 1.4 & 0.3 & 1.8 & 1.8 & 0.8 & 0.2 & $\ldots$ \\
\hline Livestock development & 0.1 & 0.4 & 0.1 & 6.7 & 4.8 & 4.4 & 0.8 \\
\hline Small-scale water project & 1.9 & $\ldots$ & $\ldots$ & $\ldots$ & $\ldots$ & & $\ldots$ \\
\hline Other & 45.7 & 67.7 & 132.4 & 91.5 & 137.6 & 100.7 & 290.5 \\
\hline Industry & 0.2 & 0.1 & 0.1 & 0.5 & 0.7 & 0.7 & 2.5 \\
\hline $\begin{array}{l}\text { Public utilities } \\
\text { Improvement of transmission/distribution }\end{array}$ & 22.7 & 25.3 & 12.4 & 5.6 & 19.7 & 16.6 & $\ldots$ \\
\hline Urban water supply & $\ldots$ & $\ldots$ & $\ldots$ & $\ldots$ & $\ldots$ & & $\ldots$ \\
\hline Rural water supply & 21.9 & 17.2 & 12.1 & 2 & 16.3 & 12.3 & $\ldots$ \\
\hline Banjul sewerage and drainage project & $\ldots$ & $\ldots$ & $\ldots$ & $\ldots$ & $\ldots$ & & $\ldots$ \\
\hline Other & 0.8 & 8.1 & 0.3 & 5.4 & 3.4 & 4.4 & $\ldots$ \\
\hline Transportation and communications & 192.1 & 223.8 & 158.3 & 186.1 & 326.7 & 600.6 & 481.8 \\
\hline Banjul Serrekunda and other highways & 152.4 & 176.4 & 104.5 & 161.5 & 91.8 & 512.2 & 453.3 \\
\hline Banjul streets rehabilitation & 0.5 & $\ldots$ & $\ldots$ & $\ldots$ & $\ldots$ & 1.0 & $\ldots$ \\
\hline Feeder roads & $\ldots$ & $\ldots$ & $\ldots$ & 10.6 & 54.8 & 3.6 & $\ldots$ \\
\hline Banjul port development & $\ldots$ & $\ldots$ & $\cdots$ & $\cdots$ & $\cdots$ & & $\ldots$ \\
\hline Yundum Airport, Phase IV & $\cdots$ & $\ldots$ & $\cdots$ & $\cdots$ & $\ldots$ & & $\ldots$ \\
\hline Other & 39.2 & 47.4 & 53.8 & 14 & 180.1 & 83.8 & 28.5 \\
\hline Education & 127.0 & 171.7 & 97.4 & 119.5 & 176.1 & 112.0 & $\ldots$ \\
\hline Health & 86.3 & 159.6 & 80.8 & 83.5 & $\ldots$ & 146.7 & $\cdots$ \\
\hline Other public investment & 26.4 & 32.7 & 93.9 & $\ldots$ & $\ldots$ & & $\ldots$ \\
\hline Housing and community development & 20.0 & 11.8 & 58.3 & 78.2 & 98.7 & 70.0 & $\ldots$ \\
\hline Tourism, trade, and finance & 0.9 & 1.5 & 6.3 & 1.6 & 18.9 & 14.9 & $\ldots$ \\
\hline General public services & 5.4 & 19.3 & 29.2 & 146.9 & 100.5 & 151.2 & $\ldots$ \\
\hline Unallocated expenditure & 0.1 & 0.1 & 0.1 & $\ldots$ & $\ldots$ & & $\ldots$ \\
\hline Total public investment financed by: & 575.2 & 745.0 & 662.0 & $\ldots$ & $\ldots$ & & $\ldots$ \\
\hline Foreign grants & 121.8 & 163.1 & 226.5 & 235.7 & 381.6 & 315.5 & $\ldots$ \\
\hline Foreign loans & 353.4 & 474.1 & 305.6 & 425 & 567.9 & 764.2 & $\ldots$ \\
\hline Gambia local fund (GLF) & 100.0 & 107.8 & 129.9 & 61 & 66.5 & 66.9 & $\ldots$ \\
\hline HIPC & & & & & 63.2 & 71.3 & \\
\hline
\end{tabular}

Source: Gambian authorities. 


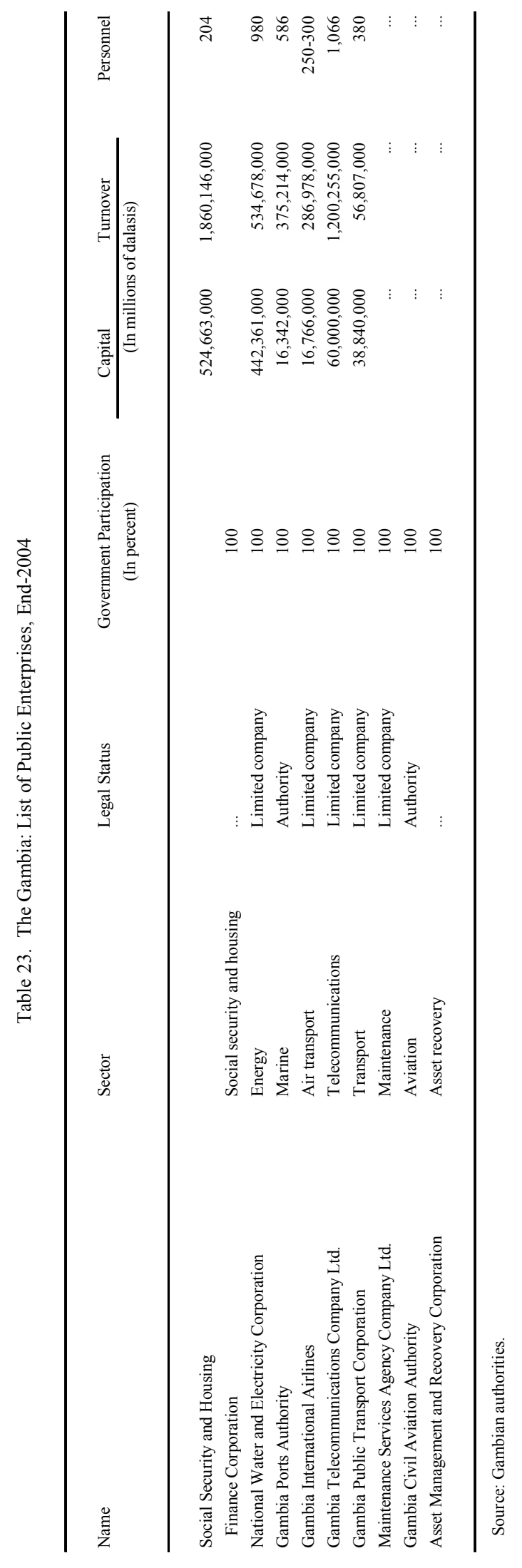


Table 24. The Gambia: Monetary Survey, December 1998-March 2005

\begin{tabular}{|c|c|c|c|c|c|c|c|c|}
\hline & $\begin{array}{c}1998 \\
\text { Dec. }\end{array}$ & $\begin{array}{c}1999 \\
\text { Dec. }\end{array}$ & $\begin{array}{c}2000 \\
\text { Dec. }\end{array}$ & $\begin{array}{c}2001 \\
\text { Dec. }\end{array}$ & $\begin{array}{c}2002 \\
\text { Dec. }\end{array}$ & $\begin{array}{c}2003 \\
\text { Dec. }\end{array}$ & $\begin{array}{c}2004 \\
\text { Dec. }\end{array}$ & $\begin{array}{l}2005 \\
\text { Mar. }\end{array}$ \\
\hline Monetary survey & \multicolumn{8}{|c|}{ (In millions of dalasis, unless otherwise noted; end of period) } \\
\hline Net foreign assets $1 /$ & 977 & 989 & 1,320 & 650 & 958 & 1,861 & 3,202 & 3,442 \\
\hline Net domestic assets & 335 & 482 & 663 & 1,717 & 2,244 & 2,732 & 2,230 & 2,506 \\
\hline Domestic credit & 508 & 661 & 770 & 2,020 & 2,699 & 3,709 & 2,870 & 3,088 \\
\hline Claims on government (net) 2/ & -7 & 38 & 83 & 589 & 612 & 1,012 & 434 & 540 \\
\hline Advances to the gov. in foreign currencies 3 / & 0 & 0 & 0 & 483 & 483 & 483 & 575 & 575 \\
\hline Claims on the private sector and public enterprises 4 / & 515 & 623 & 687 & 775 & 1,335 & 1,977 & 1,677 & 1,790 \\
\hline Claims on foreign exchange bureaus 5 / & 0 & 0 & 0 & 174 & 269 & 238 & 183 & 183 \\
\hline Other items (net) & -173 & -179 & -107 & -303 & -454 & -977 & -640 & -582 \\
\hline Broad money & 1,312 & 1,470 & 1,982 & 2,367 & 3,203 & 4,593 & 5,432 & 5,948 \\
\hline Currency outside banks & 348 & 380 & 540 & 601 & 797 & 1,183 & 1,416 & 1,535 \\
\hline Deposits & 964 & 1,091 & 1,442 & 1,767 & 2,405 & 3,410 & 4,016 & 4,412 \\
\hline \multicolumn{9}{|l|}{ Memorandum items: } \\
\hline Nominal GDP (calendar year) & $4,479.1$ & 4,922 & 5,382 & 6,556 & 7,364 & 10,026 & 12,037 & .. \\
\hline (percentage change) & 7.2 & 9.9 & 9.4 & 21.8 & 12.3 & 36.1 & 20.1 & $\ldots$ \\
\hline Velocity (calendar-year GDP/avg. broad money) & $\ldots$ & 3.6 & 3.0 & 3.2 & 2.7 & 2.6 & 2.4 & 2.2 \\
\hline \multirow[t]{2}{*}{ Reserve money multiplier (broad/reserve money) } & 2.5 & 2.4 & 2.8 & 2.8 & 2.8 & 2.5 & 2.6 & 2.6 \\
\hline & \multicolumn{8}{|c|}{ Change (in percent of beginning-of-year stocks) } \\
\hline Broad money & 10.2 & 12.1 & 34.8 & 19.4 & 35.3 & 43.4 & 18.3 & 9.5 \\
\hline Reserve money & 7.2 & 14.5 & 16.8 & 21.0 & 34.1 & 62.7 & 11.0 & 9.4 \\
\hline Total deposits & 16.2 & 13.1 & 32.2 & 22.5 & 36.2 & 41.8 & 17.8 & 9.9 \\
\hline Contribution to growth of broad money & \multicolumn{8}{|c|}{ (In percent of beginning-of-period broad money, unless otherwise noted) } \\
\hline Net foreign assets & 11.9 & 0.9 & 22.5 & -33.8 & 13.0 & 28.2 & 29.2 & 4.4 \\
\hline Net domestic assets & -1.7 & 11.2 & 12.3 & 53.2 & 22.3 & 15.2 & -10.9 & 5.1 \\
\hline Domestic credit & 6.2 & 11.7 & 7.4 & 63.1 & 28.6 & 31.5 & -18.3 & 4.0 \\
\hline Claims on government (net) $2 /$ & 0.5 & 3.4 & 3.1 & 25.5 & 1.0 & 12.5 & -12.6 & 2.0 \\
\hline Advances to the gov. in foreign currencies 3 / & 0.0 & 0.0 & 0.0 & 24.3 & 0.0 & 0.0 & 2.0 & 0.0 \\
\hline Claims on the private sector and public enterprises 4 / & 5.6 & 8.2 & 4.4 & 4.4 & 23.7 & 20.0 & -6.5 & 2.1 \\
\hline Claims on foreign exchange bureaus 5 / & 0.0 & 0.0 & 0.0 & 8.8 & 4.0 & -1.0 & -1.2 & 0.0 \\
\hline Other items (net) & -7.8 & -0.5 & 4.9 & -9.9 & -6.4 & -16.3 & 7.3 & 1.1 \\
\hline \multicolumn{9}{|c|}{ Credit to the private sector and public enterprises (excl. foreign exchange bureaus) } \\
\hline Twelve-month change (in percent) & 15.0 & 21.0 & 10.3 & 12.8 & 72.3 & 48.0 & -15.1 & 3.4 \\
\hline In percent of GDP & 11.5 & 12.6 & 12.8 & 11.8 & 18.1 & 19.7 & 13.9 & 14.3 \\
\hline \multicolumn{9}{|l|}{ Percent ratios } \\
\hline Currency/broad money & 26.5 & 25.8 & 27.3 & 25.4 & 24.9 & 25.8 & 26.1 & 25.8 \\
\hline Currency/deposits & 36.1 & 34.8 & 37.5 & 34.0 & 33.2 & 34.7 & 35.3 & 34.8 \\
\hline Deposits/broad money & 73.5 & 74.2 & 72.7 & 74.6 & 75.1 & 74.2 & 73.9 & 74.2 \\
\hline
\end{tabular}

Sources: Gambian authorities; and IMF staff estimates.

1 / Valued at current exchange rates.

2/ Excluding advances to the government in foreign currencies.

3/ These advances reflect previously unrecorded public spending and borrowing in 2001, financed by the Central Bank of The Gambia (CBG), and the previously unrecorded depletion of foreign exchange reserves in 2001-03 as reported by the authorities on October $28,2003$.

4/ In March 2003, the government instructed the CBG to lend the equivalent of D 137 million in U.S. dollars to a newly created public enterprise for a seismic survey of offshore oil deposits.

5/ Claims on foreign exchange bureaus reflect the delayed delivery of foreign currency purchased on a spot basis. 
Table 25. The Gambia: Summary Accounts of the Central Bank, December 1998-March 2005

\begin{tabular}{|c|c|c|c|c|c|c|c|c|}
\hline & $\begin{array}{r}1998 \\
\text { Dec. }\end{array}$ & $\begin{array}{c}1999 \\
\text { Dec. }\end{array}$ & $\begin{array}{c}2000 \\
\text { Dec. }\end{array}$ & $\begin{array}{c}2001 \\
\text { Dec. }\end{array}$ & $\begin{array}{c}2002 \\
\text { Dec. }\end{array}$ & $\begin{array}{c}2003 \\
\text { Dec. }\end{array}$ & $\begin{array}{r}2004 \\
\text { Dec. }\end{array}$ & $\begin{array}{r}2005 \\
\text { Mar }\end{array}$ \\
\hline & \multicolumn{8}{|c|}{ (In millions of dalasis; end of period) } \\
\hline Net foreign assets $1 /$ & 1,047 & 1,059 & 1,314 & 698 & 816 & 847 & 1,776 & 1,871 \\
\hline Foreign assets & 1,185 & 1,206 & 1,594 & 1,137 & 1,564 & 1,929 & 2,510 & 2,560 \\
\hline Foreign liabilities & -138 & -147 & -280 & -439 & -747 & $-1,081$ & -735 & -689 \\
\hline Net domestic assets & -521 & -457 & -612 & 152 & 324 & 1,008 & 285 & 382 \\
\hline Domestic credit & -537 & -573 & -778 & 139 & 381 & 1,230 & 196 & 214 \\
\hline Claims on government (net) $2 /$ & -519 & -546 & -732 & -486 & -298 & 358 & -762 & -742 \\
\hline Advances to the gov. in foreign currencies 3 / & 0 & 0 & 0 & 483 & 483 & 483 & 575 & 575 \\
\hline Claims on private sector & 21 & 22 & 23 & 24 & 24 & 25 & 29 & 29 \\
\hline Claims on banks (net) & -39 & -49 & -68 & -56 & -96 & -10 & 34 & 32 \\
\hline Claims on public enterprises 4 / & 0 & 0 & 0 & 0 & 0 & 137 & 137 & 137 \\
\hline Claims on foreign exchange bureaus 5 / & 0 & 0 & 0 & 174 & 269 & 238 & 183 & 183 \\
\hline Other items (net) & 16 & 115 & 166 & 13 & -57 & -221 & 89 & 168 \\
\hline Reserve money & 526 & 602 & 703 & 851 & 1,141 & 1,856 & 2,061 & 2,253 \\
\hline Currency outside banks & 348 & 380 & 540 & 601 & 797 & 1,183 & 1,416 & 1,535 \\
\hline Bank reserves & 178 & 222 & 162 & 250 & 343 & 673 & 644 & 718 \\
\hline Cash & 15 & 32 & 35 & 55 & 52 & 68 & 69 & 98 \\
\hline Deposits at the central bank & 163 & 190 & 127 & 195 & 291 & 605 & 575 & 620 \\
\hline Required reserves & 135 & 153 & 202 & 247 & 337 & 614 & 723 & 794 \\
\hline Excess reserves & 43 & 69 & -39 & 2 & 6 & 59 & -78 & -76 \\
\hline
\end{tabular}

Sources: Gambian authorities; and IMF staff estimates.

$1 /$ Valued at current exchange rates.

2/ Excluding advances to the government in foreign currencies.

3/ These advances reflect previously unrecorded public spending and borrowing in 2001, financed by the Central Bank of The Gambia (CBG), and previously unrecorded depletion of foreign exchange reserves in 2001-03 as reported by the authorities on October 28, 2003.

4/ In March 2003, the government instructed the CBG to lend the equivalent of D 137 million in U.S. dollars to a newly created public enterprise for a seismic survey of offshore oil deposits.

5/ Claims on foreign exchange bureaus reflect the delayed delivery of foreign currency purchased on a spot basis. 
Table 26. The Gambia: Summary Accounts of the Commercial Banks, December 1998-March 2005

\begin{tabular}{|c|c|c|c|c|c|c|c|c|}
\hline & $\begin{array}{l}1998 \\
\text { Dec. }\end{array}$ & $\begin{array}{l}1999 \\
\text { Dec. }\end{array}$ & $\begin{array}{l}2000 \\
\text { Dec. }\end{array}$ & $\begin{array}{l}2001 \\
\text { Dec. }\end{array}$ & $\begin{array}{l}2002 \\
\text { Dec. }\end{array}$ & $\begin{array}{l}2003 \\
\text { Dec. }\end{array}$ & $\begin{array}{l}2004 \\
\text { Dec. }\end{array}$ & $\begin{array}{l}2005 \\
\text { Mar. }\end{array}$ \\
\hline & \multicolumn{8}{|c|}{ (In millions of dalasis; end of period) } \\
\hline Net foreign assets 1 / & -70 & -70 & 5 & -48 & 142 & 1,014 & 1,427 & 1,571 \\
\hline Foreign assets & 72 & 126 & 137 & 128 & 568 & 1,056 & 1,507 & 1,729 \\
\hline Foreign liabilities & -142 & -196 & -132 & -176 & -426 & -42 & -81 & -158 \\
\hline Net domestic assets & 1,034 & 1,161 & 1,437 & 1,814 & 2,263 & 2,396 & 2,589 & 2,842 \\
\hline Domestic credit & 1,006 & 1,185 & 1,480 & 1,826 & 2,221 & 2,469 & 2,707 & 2,906 \\
\hline Claims on government (net) & 513 & 584 & 816 & 1,075 & 910 & 654 & 1,196 & 1,282 \\
\hline Claims on private sector and public enterprises & 490 & 591 & 664 & 751 & 1,312 & 1,815 & 1,512 & 1,623 \\
\hline Of which: in foreign currency & 0 & 0 & 0 & 95 & 95 & $\ldots$ & $\ldots$ & $\ldots$ \\
\hline Reserves & 178 & 222 & 162 & 250 & 343 & 673 & 644 & 718 \\
\hline Cash & 15 & 32 & 35 & 55 & 52 & 68 & 69 & 98 \\
\hline Deposits at the central bank & 163 & 190 & 127 & 195 & 291 & 605 & 575 & 620 \\
\hline Net claims on central bank & 39 & 49 & 68 & 56 & 96 & 10 & -34 & -32 \\
\hline Other items (net) & -189 & -294 & -274 & -317 & -398 & -756 & -729 & -750 \\
\hline Total deposit liabilities & 964 & 1,091 & 1,442 & 1,767 & 2,405 & 3,410 & 4,016 & 4,412 \\
\hline Of which : foreign currency deposits & 0 & 0 & 0 & 0 & 273 & 895 & $\ldots$ & $\ldots$ \\
\hline Demand deposits & 279 & 336 & 443 & 525 & 959 & 1,690 & 1,691 & 1,874 \\
\hline Savings deposits & 462 & 556 & 705 & 832 & 1,084 & 1,375 & 1,786 & 1,878 \\
\hline Time deposits & 223 & 198 & 293 & 410 & 362 & 345 & 538 & 660 \\
\hline
\end{tabular}

Sources: Gambian authorities; and IMF staff estimates and projections.

1/ Valued at current exchange rates. 
Table 27. The Gambia: Monthly Interest Rates on Treasury Bills, January 1998-March 2005

\begin{tabular}{|c|c|c|c|c|c|c|c|c|}
\hline & 1998 & 1999 & 2000 & 2001 & 2002 & 2003 & 2004 & 2005 \\
\hline & \multicolumn{8}{|c|}{ (In percent per annum) } \\
\hline January & 16.0 & 14.0 & 12.5 & 12.0 & 15.0 & 20.0 & 31.0 & 28.0 \\
\hline February & 16.0 & 14.0 & 12.5 & 12.5 & 15.0 & 20.0 & 31.0 & 28.0 \\
\hline March & 16.0 & 14.0 & 12.0 & 12.5 & 15.0 & 23.0 & 31.0 & 26.0 \\
\hline April & 16.0 & 14.0 & 12.0 & 12.5 & 15.0 & 24.0 & 31.0 & ... \\
\hline May & 16.0 & 14.0 & 12.0 & 12.5 & 15.0 & 24.0 & 31.0 & $\ldots$ \\
\hline June & 16.0 & 14.0 & 12.0 & 12.5 & 15.0 & 25.0 & 31.0 & $\ldots$ \\
\hline July & 16.0 & 13.5 & 12.0 & 12.5 & 15.0 & 26.0 & 31.0 & ... \\
\hline August & 16.0 & 13.5 & 12.0 & 12.5 & 15.0 & 31.0 & 31.0 & ... \\
\hline September & 16.0 & 13.5 & 12.0 & 12.5 & 15.0 & 31.0 & 31.0 & ... \\
\hline October & 15.5 & 13.5 & 12.0 & 15.0 & 18.0 & 31.0 & 30.0 & $\ldots$ \\
\hline November & 14.5 & 13.5 & 12.0 & 15.0 & 19.0 & 31.0 & 30.0 & ... \\
\hline December & 14.0 & 12.5 & 12.0 & 15.0 & 20.0 & 31.0 & 30.0 & $\ldots$ \\
\hline
\end{tabular}

Source: Central Bank of The Gambia. 
Table 28. The Gambia: Structure of Interest Rates, 1998-March 2005 1/

\begin{tabular}{|c|c|c|c|c|c|c|c|c|}
\hline & 1998 & 1999 & 2000 & 2001 & 2002 & 2003 & 2004 & $\begin{array}{c}\text { March } \\
2005\end{array}$ \\
\hline & \multicolumn{8}{|c|}{ (In percent per annum; end of period) } \\
\hline \multicolumn{9}{|c|}{ Commercial bank lending rates 2 / } \\
\hline Agriculture & $19.0-24.0$ & $18.0-24.0$ & $18.0-24.0$ & $18.0-24.0$ & $17.0-24.0$ & $21.0-36.5$ & $21.0-36.5$ & $21.0-36.0$ \\
\hline Manufacturing & $19.0-24.0$ & $18.0-24.0$ & $18.0-24.0$ & $18.0-24.0$ & $17.0-24.0$ & $21.0-36.5$ & $21.0-36.5$ & $21.0-36.0$ \\
\hline Building & $19.0-24.0$ & $18.0-24.0$ & $18.0-24.0$ & $18.0-24.0$ & $17.0-24.0$ & $21.0-36.5$ & $21.0-36.5$ & $21.0-36.0$ \\
\hline Trading & $19.0-24.0$ & $18.0-24.0$ & $18.0-24.0$ & $18.0-24.0$ & $17.0-24.0$ & $21.0-36.5$ & $21.0-36.5$ & $21.0-36.0$ \\
\hline Tourism & $19.0-24.0$ & $18.0-24.0$ & $18.0-24.0$ & $18.0-24.0$ & $17.0-24.0$ & $21.0-36.5$ & $21.0-36.5$ & $21.0-36.0$ \\
\hline Other & $19.0-24.0$ & $18.0-24.0$ & $18.0-24.0$ & $18.0-24.0$ & $17.0-24.0$ & $21.0-36.5$ & $21.0-36.5$ & $21.0-36.0$ \\
\hline \multicolumn{9}{|l|}{ Deposits } \\
\hline Short-term deposit account & 9.0 & 7.0 & 7.0 & 7.0 & 7.0 & 6.5 & 6.5 & 6.5 \\
\hline Savings bank account & $9.5-11.5$ & $9.0-10.0$ & $8.0-10.0$ & $8.0-10.0$ & $8.0-10.0$ & $8.0-17.0$ & $10.0-17.0$ & $10.0-17.0$ \\
\hline \multicolumn{9}{|l|}{ Time deposits } \\
\hline Three months & $11.5-12.5$ & $9.5-12.5$ & $9.5-12.5$ & $9.5-12.5$ & $6.0-13.0$ & $7.0-22.0$ & $8.0-22.0$ & $8.0-18.0$ \\
\hline Six months & $11.5-12.5$ & $10.2-12.5$ & $10.0-12.5$ & $10.0-12.5$ & $6.0-13.0$ & $8.0-22.0$ & $8.0-22.0$ & $8.0-19.0$ \\
\hline Nine months & $12.0-15.0$ & $11.0-12.5$ & $10.8-12.5$ & $10.8-12.5$ & $7.0-13.0$ & $8.0-22.0$ & $8.0-22.0$ & $8.0-20.0$ \\
\hline Twelve months and over & $12.0-15.0$ & $12.0-12.5$ & $11.0-12.5$ & $11.0-12.5$ & $7.0-13.0$ & $10.0-22.0$ & $12.0-23.0$ & $12.0-23.0$ \\
\hline \multicolumn{9}{|l|}{ Government 3/ } \\
\hline Treasury bills & 14.0 & 12.5 & 12.0 & 15.0 & 20.0 & 31.0 & 30.0 & 26.0 \\
\hline \multicolumn{9}{|c|}{ Government development loans } \\
\hline 1994-99 & 15.5 & 15.5 & 15.0 & 15.0 & 15.0 & $\ldots$ & $\ldots$ & $\ldots$ \\
\hline $1999-2002$ & $\ldots$ & 14.0 & 14.0 & 14.0 & 14.0 & $\ldots$ & $\ldots$ & $\ldots$ \\
\hline 2002 & $\ldots$ & $\ldots$ & $\ldots$ & $\ldots$ & $\ldots$ & $15.5-20.0$ & $15.5-20.0$ & $15.5-20.0$ \\
\hline \multicolumn{9}{|l|}{ Central bank } \\
\hline Bank rate & 12.0 & 10.5 & 10.0 & 13.0 & 18.0 & 29.0 & 28.0 & 24.0 \\
\hline \multicolumn{9}{|l|}{ Secondary market operations } \\
\hline Rediscount rate & 17.0 & 15.5 & 15.0 & 18.0 & 23.0 & 34.0 & 33.0 & 29.0 \\
\hline
\end{tabular}

Source: Central Bank of The Gambia.

1/ Until 1996/97, fiscal years (July-June); from 1997, calendar years.

2/ One commercial bank retains historical nonperforming loans on its books that were made at 9 percent.

3/ Three-month treasury bill rate (end-of-period). 


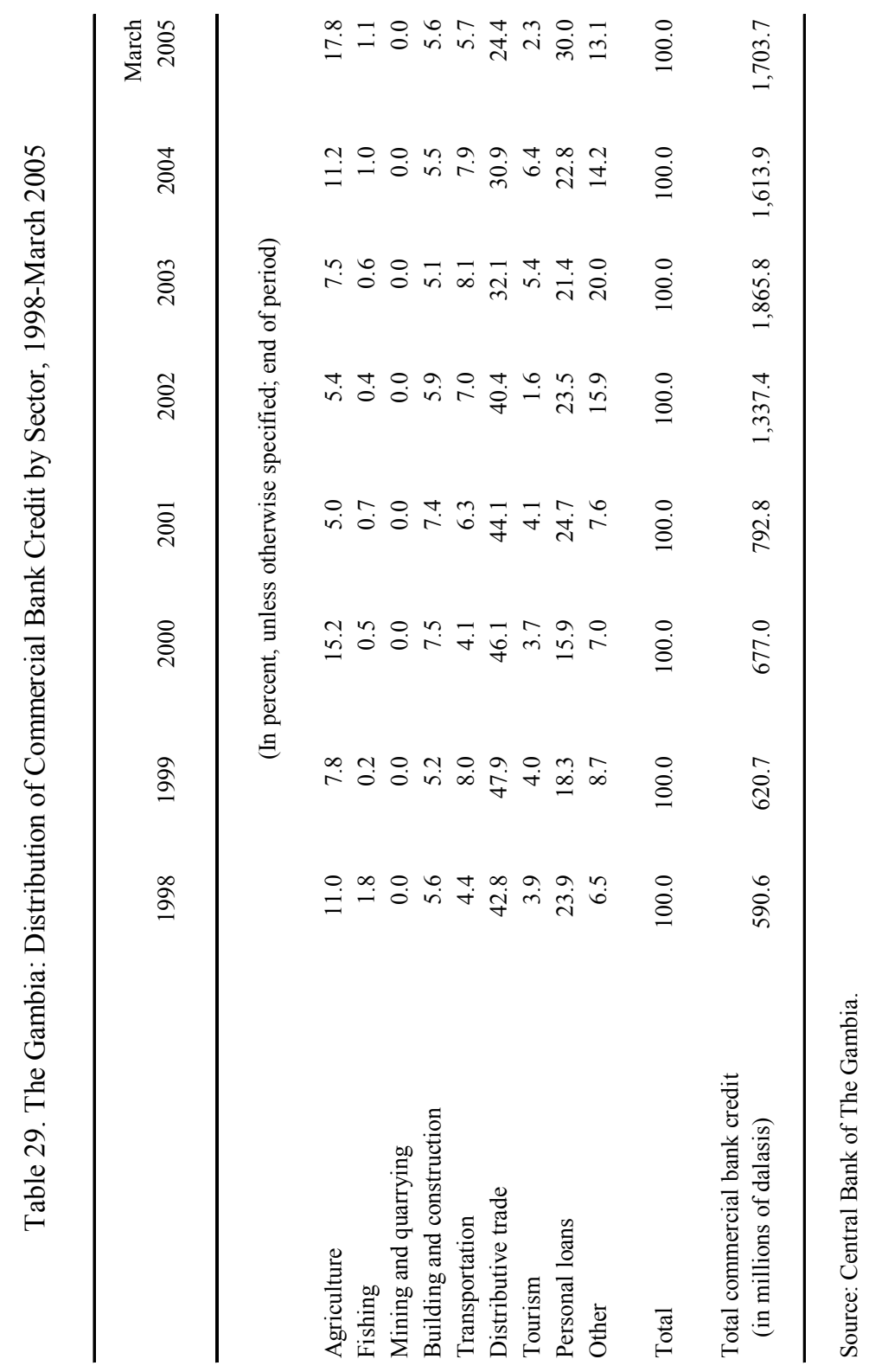




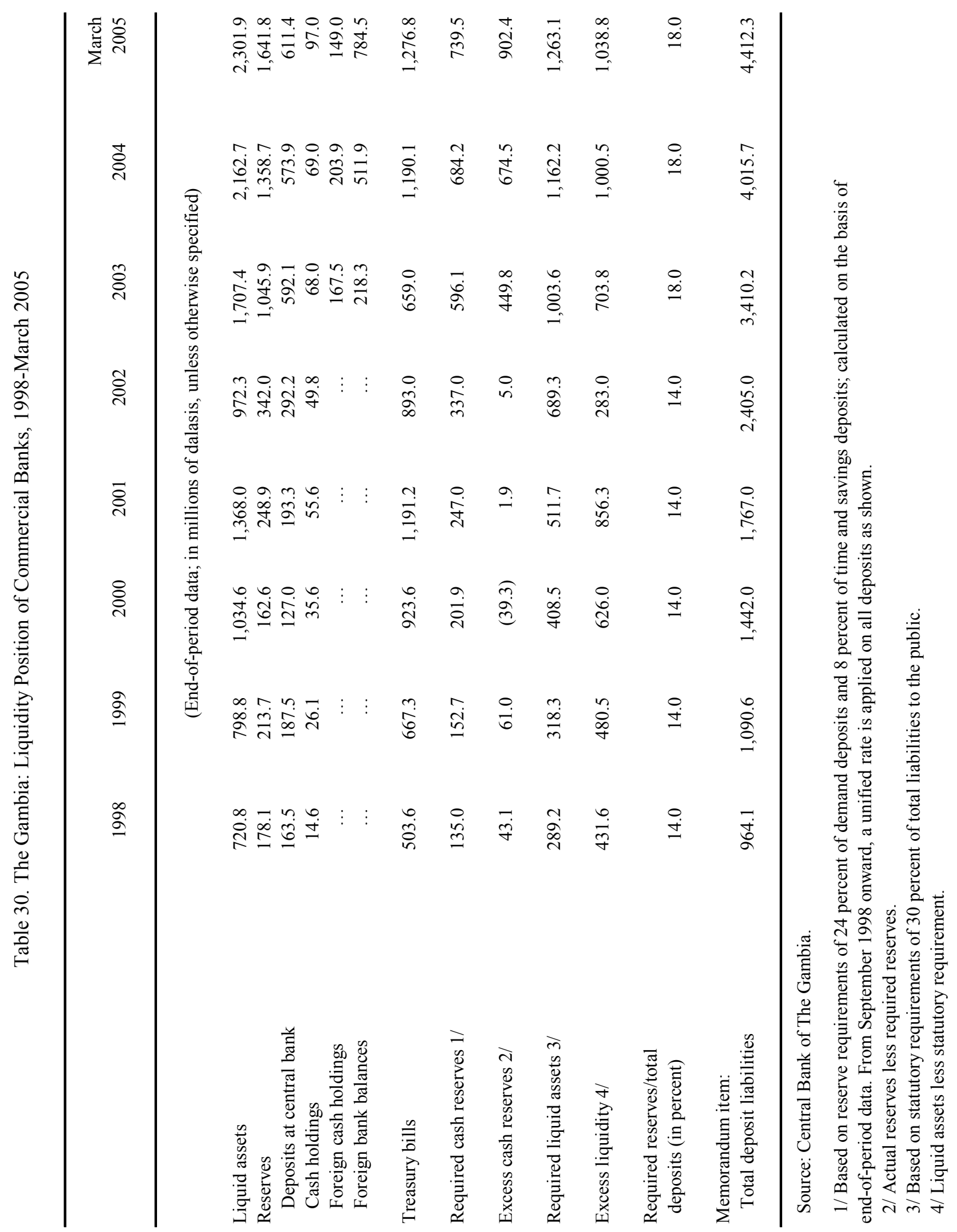




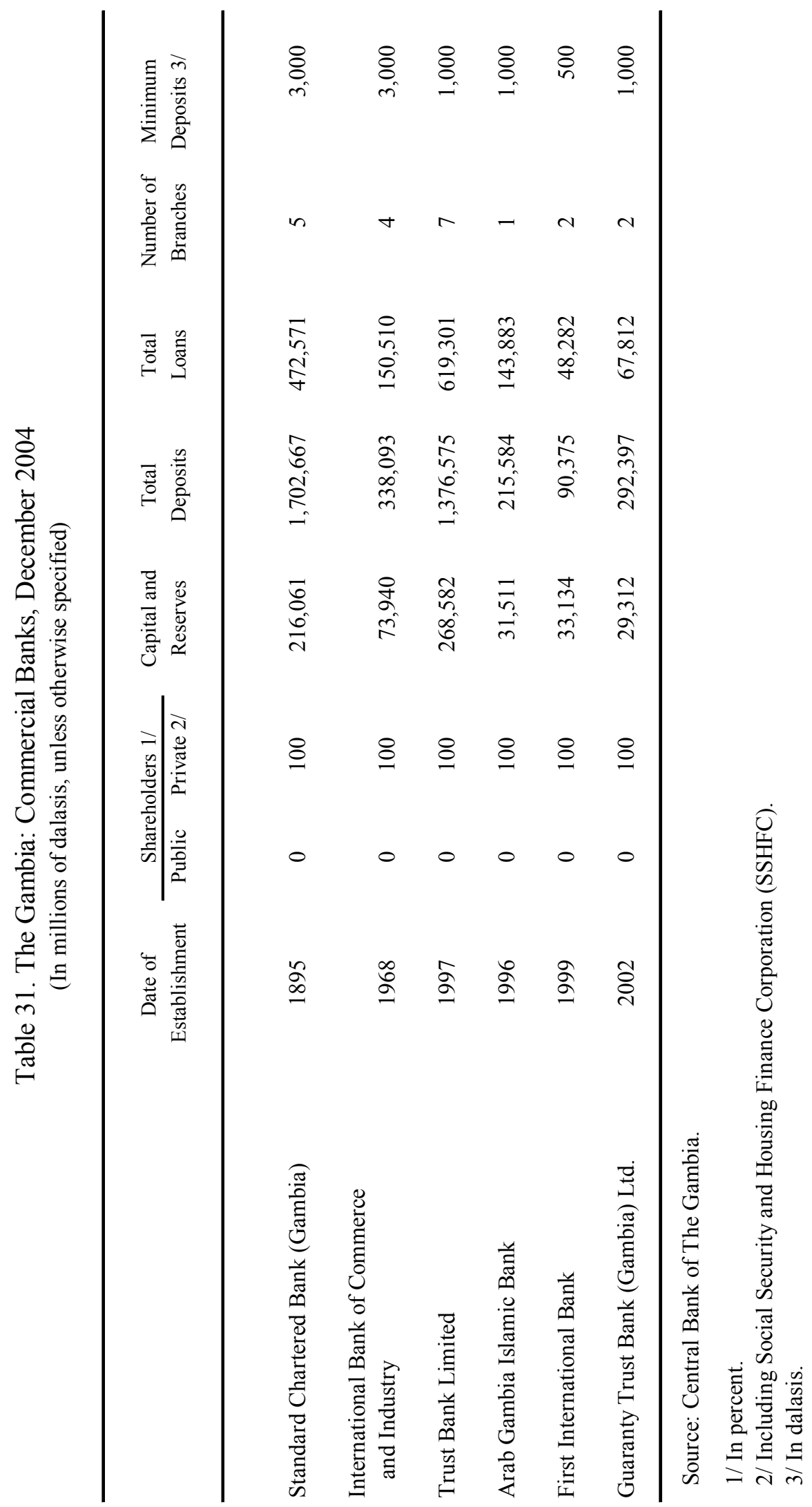




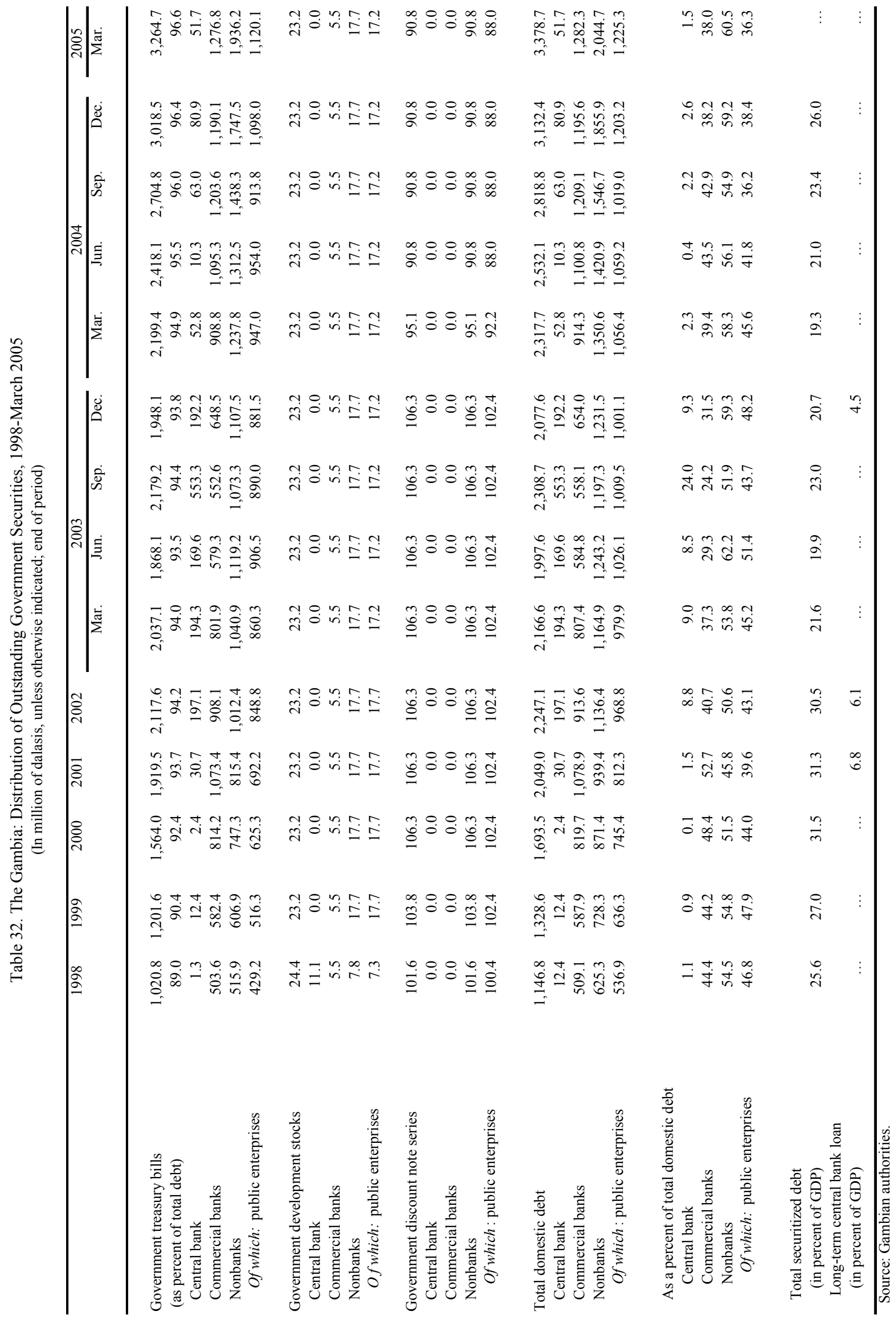


Table 33. The Gambia: Balance of Payments, 1998-2004

(In millions of U.S. dollars, unless otherwise indicated)

\begin{tabular}{|c|c|c|c|c|c|c|c|}
\hline & 1998 & 1999 & 2000 & 2001 & 2002 & $\begin{array}{c}2003 \\
\text { Prel. }\end{array}$ & $\begin{array}{c}2004 \\
\text { Prel }\end{array}$ \\
\hline Trade balance & -78.9 & -68.7 & -63.3 & -50.0 & -62.4 & -60.0 & -108.4 \\
\hline Exports, f.o.b. & 130.4 & 120.2 & 126.6 & 102.1 & 109.3 & 101.0 & 127.0 \\
\hline Groundnuts/groundnut products & 12.5 & 9.9 & 13.7 & 18.0 & 24.0 & 9.1 & 16.9 \\
\hline Other domestic exports & 8.6 & 5.9 & 7.4 & 8.3 & 7.1 & 8.4 & 9.0 \\
\hline Reexports & 109.3 & 104.4 & 105.4 & 75.8 & 78.2 & 83.5 & 101.2 \\
\hline Imports, c.i.f. & -209.3 & -188.9 & -189.9 & -152.1 & -171.6 & -161.0 & -235.4 \\
\hline For domestic use & -124.0 & -111.4 & -113.6 & -96.2 & -114.0 & -100.2 & -165.7 \\
\hline Of which: oil products & -13.7 & -10.5 & -22.6 & -9.2 & -16.7 & -16.0 & -33.8 \\
\hline For reexport & -85.3 & -77.5 & -76.3 & -55.9 & -57.7 & -60.7 & -69.7 \\
\hline Factor services (net) & -20.0 & -21.1 & -20.5 & -23.1 & -22.4 & -19.1 & -19.9 \\
\hline Nonfactor services & 49.7 & 43.8 & 28.7 & 27.4 & 31.6 & 27.4 & 37.7 \\
\hline Of which: travel income & 69.2 & 63.8 & 47.7 & 47.9 & 47.7 & 51.1 & 57.5 \\
\hline Private unrequited transfers (net) & 3.5 & 3.8 & 10.6 & 3.5 & 3.6 & 3.7 & 3.9 \\
\hline Official unrequited transfers (net) & 35.6 & 30.0 & 31.3 & 31.4 & 39.2 & 30.0 & 39.6 \\
\hline \multicolumn{8}{|l|}{ Current account balance } \\
\hline Excluding official transfers & -45.7 & -42.2 & -44.5 & -42.2 & -49.6 & -48.0 & -86.7 \\
\hline Including official transfers & -10.1 & -12.2 & -13.2 & -10.8 & -10.4 & -18.0 & -47.1 \\
\hline Capital account & 17.3 & 7.5 & 6.0 & 22.8 & 9.9 & 6.7 & 68.0 \\
\hline Official loans (net) & 5.4 & 2.7 & 6.6 & 8.4 & 18.9 & 16.1 & 24.9 \\
\hline Project related & 14.2 & 13.7 & 20.7 & 24.8 & 47.4 & 26.5 & 39.2 \\
\hline Amortization & -8.8 & -11.0 & -14.1 & -16.4 & -29.7 & -10.3 & -14.4 \\
\hline \multicolumn{8}{|l|}{ Private capital inflow } \\
\hline Foreign direct investment (net) & 15.6 & 0.4 & 15.8 & 10.2 & 12.9 & 12.7 & 49.7 \\
\hline Other investment (net) & -3.6 & 4.4 & -16.4 & 4.3 & -22.0 & -22.1 & -6.5 \\
\hline Of which: suppliers' credits & 3.6 & 3.3 & 3.3 & 16.9 & -12.3 & 4.3 & 6.3 \\
\hline Unaccounted-for loss in official reserves & 0.0 & 0.0 & 0.0 & -28.5 & 0.5 & 0.0 & 0.0 \\
\hline Errors and omissions & -0.8 & -0.6 & 17.4 & -39.6 & -0.1 & 6.3 & 12.6 \\
\hline Overall balance (excl. PRSP) & 6.5 & -5.3 & 10.1 & -56.1 & -0.1 & -4.9 & 33.5 \\
\hline Financing & -6.5 & 5.3 & -10.1 & 56.1 & 0.1 & 4.9 & -33.5 \\
\hline Change in gross official reserves (increase $=-$ ) & -5.5 & 4.8 & -16.9 & 47.1 & -4.2 & 4.9 & -22.3 \\
\hline \multicolumn{8}{|l|}{ Of which } \\
\hline Repurchases/repayments (IMF) & -4.9 & -3.5 & -1.6 & -0.2 & 0.0 & 0.0 & -11.2 \\
\hline Purchases/loans (IMF) & 4.7 & 4.7 & 9.1 & 8.7 & 3.7 & 0.0 & 0.0 \\
\hline Exceptional financing 1/ & 0.0 & 0.0 & 0.0 & 0.0 & 0.4 & 0.0 & 0.0 \\
\hline \multicolumn{8}{|l|}{ Memorandum items: } \\
\hline \multicolumn{8}{|l|}{ Current account balance (in percent of GDP) } \\
\hline Excluding official transfers & -10.9 & -9.8 & -10.6 & -10.1 & -13.4 & -13.6 & -21.6 \\
\hline Including official transfers & -2.4 & -2.8 & -3.1 & -2.6 & -2.8 & -5.1 & -11.8 \\
\hline \multicolumn{8}{|l|}{ Gross official reserves (end of period) } \\
\hline In millions of U.S. dollars & 102.0 & 98.0 & 111.4 & 63.0 & 67.2 & 62.3 & 84.6 \\
\hline In months of imports, c.i.f. & 5.8 & 6.2 & 7.0 & 5.0 & 4.5 & 4.4 & 4.1 \\
\hline \multicolumn{8}{|l|}{ In months of imports, c.i.f. } \\
\hline Over the next 12 months & 6.5 & 6.0 & 8.5 & 4.5 & 5.2 & 5.2 & 3.2 \\
\hline Plus all other services payments & 4.8 & 4.4 & 5.9 & 3.3 & 3.6 & 4.4 & 2.4 \\
\hline \multicolumn{8}{|l|}{ External debt-service ratio $2 /$} \\
\hline Including the Fund & 11.1 & 11.8 & 15.4 & 16.4 & 16.9 & 8.5 & 15.9 \\
\hline Excluding the Fund & 8.4 & 9.6 & 14.3 & 16.3 & 16.8 & 8.4 & 9.7 \\
\hline Nominal GDP & 420.8 & 431.9 & 420.9 & 417.9 & 369.7 & 353.0 & 400.8 \\
\hline Nominal GDP (in millions of dalasis) & $4,479.1$ & $4,921.9$ & $5,382.4$ & $6,555.9$ & $7,364.3$ & $10,025.9$ & 12036.6 \\
\hline Official rate (average) & 10.64 & 11.40 & 12.79 & 15.69 & 19.92 & 28.40 & 30.0 \\
\hline
\end{tabular}

Sources: Gambian authorities; and IMF staff estimates.

1/ Includes debt relief by Paris Club; interim relief by multilaterals is treated as grants

2/ As a percentage of exports and travel income. After interim debt relief and HIPC grants. 


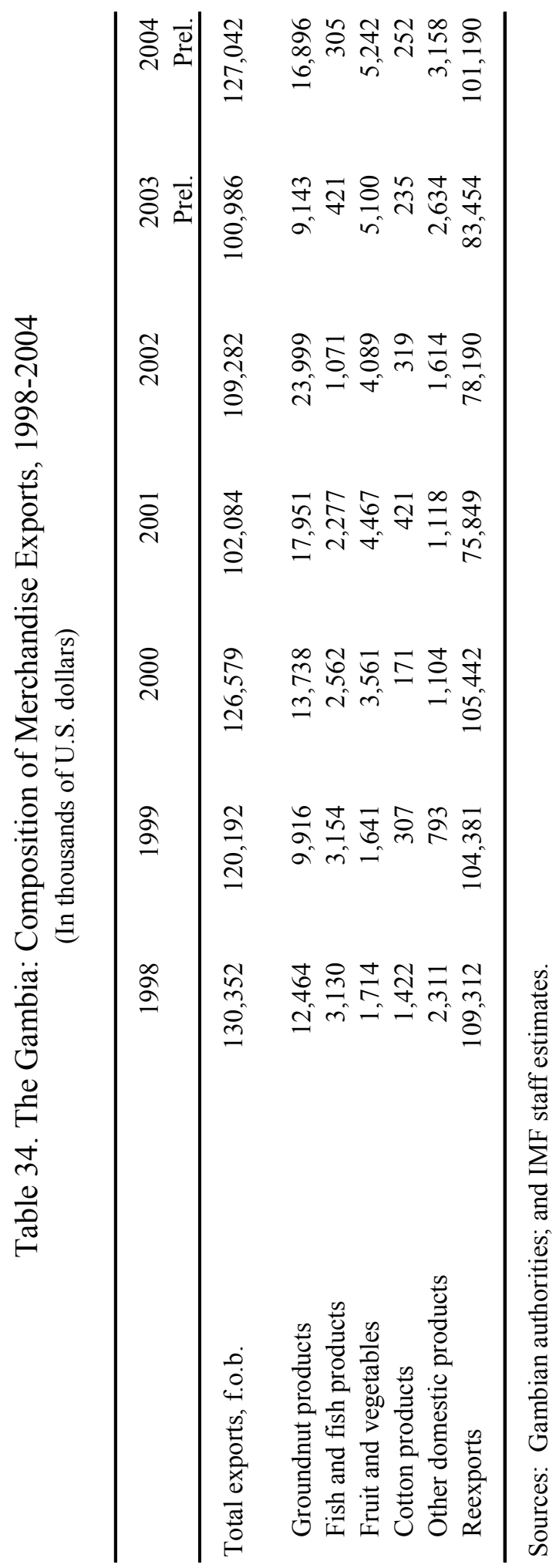


Table 35. The Gambia: Composition of Merchandise Imports, 1998-2004 (In thousands of U.S. dollars)

\begin{tabular}{|c|c|c|c|c|c|c|c|}
\hline & 1998 & 1999 & 2000 & 2001 & 2002 & 2003 & 2004 \\
\hline Total imports, c.i.f. 1/ & 209,203 & 189,944 & 189,858 & 144,659 & 160,105 & 152,607 & 236,604 \\
\hline Food and live animals & 71,096 & 61,721 & 59,800 & 29,642 & 37,581 & 40,456 & 64,687 \\
\hline Beverages and tobacco & 6,989 & 7,288 & 4,527 & 13,782 & 8,231 & 8,592 & 12,374 \\
\hline Raw materials & 2,496 & 3,203 & 1,526 & 3,799 & 7,188 & 3,922 & 5,134 \\
\hline $\begin{array}{l}\text { Minerals, fuel, lubricants, and } \\
\text { related materials }\end{array}$ & 13,418 & 10,640 & 22,627 & 9,160 & 17,008 & 21,777 & 23,921 \\
\hline Animal and vegtable oils & 9,277 & 5,856 & 6,023 & 7,252 & 6,078 & 6,104 & 11,404 \\
\hline Chemicals & 12,324 & 14,133 & 13,154 & 8,701 & 16,201 & 9,965 & 17,414 \\
\hline $\begin{array}{l}\text { Manufactured goods classified } \\
\text { by material }\end{array}$ & 22,652 & 22,272 & 23,162 & 17,823 & 24,294 & 18,481 & 30,617 \\
\hline Machinery and transport equipment & 47,234 & 39,034 & 35,820 & 36,755 & 27,262 & 26,325 & 42,778 \\
\hline Miscellaneous manufactured articles & 19,497 & 23,308 & 21,561 & 12,557 & 15,563 & 14,559 & 24,536 \\
\hline $\begin{array}{l}\text { Commodities and transactions not } \\
\text { classified according to kind }\end{array}$ & 4,222 & 2,489 & 1,660 & 5,188 & 700 & 2,426 & 3,738 \\
\hline
\end{tabular}

Source: Gambian authorities.

1/ Data do not include the imports of three generators in 2001, and some of the imports of Youth Development Enterprise (YDE) during 2001-2003. Data for 2004 are converted from the Harmonized System codes. 
Table 36. The Gambia: Direction of Trade, 1998-2004 1/

(In percent of total)

\begin{tabular}{|c|c|c|c|c|c|c|c|}
\hline & 1998 & 1999 & 2000 & 2001 & 2002 & 2003 & 2004 \\
\hline Exports & 100.0 & 100.0 & 100.0 & 100.0 & 100.0 & 100.0 & 100.0 \\
\hline Industrial countries & 81.9 & 75.1 & 76.7 & 78.9 & 78.0 & 47.6 & 56.5 \\
\hline \multicolumn{8}{|l|}{ Of which } \\
\hline United States & 0.4 & 3.2 & 0.7 & 2.1 & 1.0 & 0.0 & 1.2 \\
\hline Japan & 1.7 & 2.2 & 8.0 & 2.1 & 0.5 & 1.6 & 2.2 \\
\hline EU-15 & 80.1 & 70.7 & 67.7 & 67.0 & 76.3 & 46.2 & 53.2 \\
\hline Developing countries & 18.1 & 24.9 & 23.3 & 21.1 & 22.0 & 52.4 & 43.5 \\
\hline \multicolumn{8}{|l|}{ Of which } \\
\hline Africa & 10.9 & 18.1 & 8.5 & 7.9 & 4.8 & 7.5 & 5.6 \\
\hline \multicolumn{8}{|l|}{ Of which } \\
\hline Ghana & 1.0 & 1.8 & 0.4 & 0.7 & 0.7 & 1.4 & 0.9 \\
\hline Guinea & 2.5 & 4.2 & 0.2 & 0.1 & 0.1 & 0.2 & 0.1 \\
\hline Senegal & 2.3 & 6.4 & 0.3 & 0.0 & 0.6 & 0.1 & 0.0 \\
\hline Guinea Bissau & 2.5 & 2.8 & 0.6 & 1.1 & 1.2 & 2.2 & 1.4 \\
\hline Asia & 3.3 & 5.8 & 6.5 & 10.1 & 16.2 & 41.2 & 36.5 \\
\hline \multicolumn{8}{|l|}{ Of which } \\
\hline China & 2.2 & 1.5 & 0.1 & 0.0 & 0.0 & 7.7 & 0.5 \\
\hline Hong Kong SAR & 0.5 & 0.8 & 1.8 & 0.9 & 1.0 & 1.9 & 0.3 \\
\hline Thailand & 0.2 & 0.2 & 0.8 & 3.1 & 0.6 & 3.2 & 16.5 \\
\hline Other & 3.9 & 1.1 & 8.3 & 3.0 & 1.1 & 3.8 & 1.4 \\
\hline Imports & 100.0 & 100.0 & 100.0 & 100.0 & 100.0 & 100.0 & 100.0 \\
\hline Industrial countries & 42.3 & 57.4 & 35.0 & 39.1 & 35.3 & 34.9 & 29.1 \\
\hline \multicolumn{8}{|l|}{ Of which } \\
\hline United States & 3.2 & 5.0 & 2.5 & 2.3 & 2.5 & 5.8 & 4.4 \\
\hline Japan & 2.2 & 3.2 & 2.0 & 1.3 & 1.6 & 1.1 & 0.6 \\
\hline EU-15 & 36.6 & 50.3 & 30.1 & 35.2 & 30.5 & 27.9 & 24.0 \\
\hline Developing countries & 57.6 & 42.6 & 65.0 & 60.9 & 64.7 & 65.1 & 70.9 \\
\hline \multicolumn{8}{|l|}{ Of which } \\
\hline Africa & 11.7 & 8.7 & 17.1 & 14.2 & 16.7 & 15.9 & 18.8 \\
\hline \multicolumn{8}{|l|}{ Of which } \\
\hline Guinea & 0.4 & 0.0 & 0.0 & 0.0 & 0.0 & 0.0 & 0.0 \\
\hline Senegal & 5.4 & 2.3 & 10.9 & 7.4 & 8.8 & 9.0 & 10.4 \\
\hline Côte d'Ivoire & 4.5 & 4.1 & 3.9 & 3.3 & 4.8 & 3.5 & 4.0 \\
\hline Asia & 39.0 & 20.3 & 38.3 & 34.3 & 35.6 & 36.6 & 37.3 \\
\hline \multicolumn{8}{|l|}{ Of which } \\
\hline China & 17.0 & 6.4 & 20.3 & 20.0 & 21.6 & 25.2 & 24.6 \\
\hline Hong Kong SAR & 11.5 & 4.4 & 8.6 & 4.3 & 3.4 & 3.0 & 2.3 \\
\hline Thailand & 1.0 & 1.2 & 1.0 & 2.1 & 1.3 & 0.8 & 1.0 \\
\hline Other & 6.9 & 13.6 & 9.5 & 12.4 & 12.3 & 12.7 & 14.8 \\
\hline
\end{tabular}

Source: IMF, Direction of Trade Statistics.

1/ Based on The Gambia's trading partners' data. 
Table 37. The Gambia: Foreign Trade Indicators, 1998-2004

\begin{tabular}{|c|c|c|c|c|c|c|c|}
\hline & 1998 & 1999 & 2000 & 2001 & 2002 & 2003 & 2004 \\
\hline & \multicolumn{7}{|c|}{ (Annual percentage changes) } \\
\hline \multicolumn{8}{|l|}{ Value (U.S. dollar terms) } \\
\hline Exports, f.o.b. & 19.9 & -7.8 & 5.3 & -19.4 & 7.1 & -7.6 & 25.8 \\
\hline Groundnuts 1/ & 158.3 & -20.4 & 38.6 & 30.7 & 33.7 & -61.9 & 84.8 \\
\hline Other domestic exports & -6.7 & -31.3 & 25.5 & 12.0 & -14.4 & 18.3 & 6.8 \\
\hline Reexports & 15.4 & -4.5 & 1.0 & -28.1 & 3.1 & 6.7 & 21.3 \\
\hline Travel income & 9.2 & -7.8 & -25.1 & 0.3 & -0.3 & 7.1 & 12.4 \\
\hline Imports, c.i.f. & 20.3 & -9.7 & 0.5 & -19.9 & 12.8 & -6.2 & 46.2 \\
\hline For domestic use & 17.6 & -10.1 & 2.0 & -15.3 & 18.5 & -12.1 & 65.3 \\
\hline Of which: oil products & 12.0 & -23.4 & 116.2 & -59.5 & 82.4 & -4.2 & 110.5 \\
\hline For reexports & 24.5 & -9.2 & -1.5 & -26.7 & 3.1 & 5.3 & 14.8 \\
\hline \multicolumn{8}{|l|}{ Price indices (U.S. dollar terms) } \\
\hline Exports, f.o.b. & -2.0 & -6.3 & -5.1 & -2.4 & 5.3 & 15.3 & 8.8 \\
\hline Groundnuts 1/ & -9.1 & -11.9 & -2.9 & -17.1 & 39.1 & -11.5 & 50.5 \\
\hline Other domestic exports & -0.4 & -21.0 & 8.1 & 8.4 & 8.0 & 18.0 & 8.1 \\
\hline Reexports & -10.1 & 0.7 & -3.3 & -4.1 & 3.1 & 12.3 & 13.9 \\
\hline Travel income & 1.0 & -30.7 & 17.7 & 3.0 & -4.6 & -5.1 & 11.2 \\
\hline Imports, c.i.f. & -5.0 & -1.6 & -0.7 & -3.1 & 3.0 & 11.3 & 12.2 \\
\hline For domestic use & -6.3 & 2.0 & 2.7 & -3.2 & 3.0 & 11.5 & 14.8 \\
\hline Of which: oil products & -32.1 & 37.5 & 57.0 & -13.8 & 2.5 & 15.8 & 48.4 \\
\hline For reexports & -3.1 & -4.2 & -5.7 & -2.2 & 3.1 & 10.9 & 7.8 \\
\hline \multicolumn{8}{|l|}{ Volume indices } \\
\hline Exports, f.o.b. & 22.4 & -1.6 & 11.0 & -17.4 & 1.7 & -19.8 & 15.6 \\
\hline Groundnuts 1/ & 184.2 & -9.7 & 42.7 & 57.7 & -3.9 & -57.0 & 22.8 \\
\hline Other domestic exports & -6.3 & -13.0 & 16.0 & 3.3 & -20.7 & 0.3 & -1.3 \\
\hline Reexports & 28.4 & -5.2 & 4.4 & -25.0 & 0.0 & -5.0 & 6.5 \\
\hline Travel income & 8.1 & 33.0 & -36.4 & -2.6 & 4.5 & 12.9 & 1.1 \\
\hline Imports, c.i.f. & 26.7 & -8.3 & 1.2 & -17.3 & 9.5 & -15.8 & 30.4 \\
\hline For domestic use & 25.6 & -11.9 & -0.8 & -12.5 & 15.0 & -21.2 & 43.9 \\
\hline Of which: oil products & 65.0 & -44.3 & 37.7 & -52.9 & 77.8 & -17.3 & 41.9 \\
\hline For reexports & 28.4 & -5.2 & 4.4 & -25.0 & 0.0 & -5.0 & 6.5 \\
\hline \multicolumn{8}{|l|}{ Terms of trade } \\
\hline Domestic trade 2/ & 11.8 & -18.0 & -3.5 & 0.7 & 8.3 & 18.2 & -10.2 \\
\hline Total trade & 3.1 & -4.9 & -4.4 & 0.8 & 2.2 & 5.0 & -4.7 \\
\hline & \multicolumn{7}{|c|}{ (In percent of GDP) } \\
\hline Exports, f.o.b. & 31.0 & 27.8 & 30.1 & 24.4 & 29.6 & 28.6 & 31.7 \\
\hline Groundnuts 1/ & 3.0 & 2.3 & 3.3 & 4.3 & 6.5 & 2.6 & 4.2 \\
\hline Other domestic exports & 2.0 & 1.4 & 1.8 & 2.0 & 1.9 & 2.4 & 2.2 \\
\hline Reexports & 26.0 & 24.2 & 25.1 & 18.1 & 21.1 & 23.6 & 25.2 \\
\hline Travel income & 16.4 & 14.8 & 11.3 & 11.5 & 12.9 & 14.5 & 14.3 \\
\hline Imports, c.i.f. & 49.7 & 43.7 & 45.1 & 36.4 & 46.4 & 45.6 & 58.7 \\
\hline Of which: for domestic use & 29.5 & 25.8 & 27.0 & 23.0 & 30.8 & 28.4 & 41.3 \\
\hline
\end{tabular}

Sources: Gambian authorities; and IMF staff estimates.

1/ Including undecorticated groundnut exports.

2/ Pertains to exports of domestically produced goods. 
Table 38. The Gambia: Public External Debt Outstanding and Debt Service, 1998-2004 (In millions of U.S. dollars)

\begin{tabular}{|c|c|c|c|c|c|c|c|}
\hline & 1998 & 1999 & 2000 & 2001 & 2002 & 2003 & $\begin{array}{r}2004 \\
\text { Prel }\end{array}$ \\
\hline External public debt & 460.5 & 451.5 & 454.3 & 458.3 & 481.1 & 511.6 & 580.7 \\
\hline Medium and long term & 450.4 & 440.0 & 436.0 & 432.1 & 450.7 & 478.8 & 557.1 \\
\hline IMF & 10.0 & 11.5 & 18.4 & 26.2 & 30.4 & 32.9 & 23.6 \\
\hline External public debt service 1/ & 22.8 & 21.8 & 26.8 & 29.4 & 34.7 & 16.9 & 34.4 \\
\hline Principal & 16.8 & 15.6 & 21.8 & 24.5 & 30.1 & 10.9 & 26.3 \\
\hline Medium and long term & 8.8 & 12.1 & 20.2 & 24.2 & 30.1 & 10.9 & 15.1 \\
\hline IMF repayments & 4.9 & 3.5 & 1.6 & 0.2 & 0.0 & 0.0 & 11.2 \\
\hline Interest & 6.0 & 6.1 & 5.0 & 4.9 & 4.6 & 6.0 & 8.1 \\
\hline Medium and long term & 5.6 & 5.6 & 4.7 & 4.5 & 4.3 & 5.9 & 7.9 \\
\hline Short term & 0.0 & 0.0 & 0.0 & 0.0 & 0.0 & 0.0 & 0.0 \\
\hline IMF interest & 0.4 & 0.5 & 0.3 & 0.4 & 0.3 & 0.1 & 0.2 \\
\hline IMF & 5.3 & 4.0 & 1.9 & 0.6 & 0.3 & 0.1 & 11.4 \\
\hline Others & 17.5 & 17.7 & 24.9 & 28.7 & 34.5 & 16.8 & 23.0 \\
\hline \multicolumn{8}{|l|}{ Memorandum items: } \\
\hline $\begin{array}{l}\text { External public debt } \\
\text { (in percent of GDP) }\end{array}$ & 109.4 & 104.6 & 107.9 & 109.7 & 130.1 & 144.9 & 144.9 \\
\hline \multicolumn{8}{|l|}{ Debt service } \\
\hline (in percent of GDP) & 5.4 & 5.0 & 6.4 & 7.0 & 9.4 & 4.8 & 8.6 \\
\hline Debt-service ratio 2/ & 11.4 & 11.8 & 15.4 & 19.6 & 22.1 & 11.1 & 18.6 \\
\hline IMF & 2.7 & 2.2 & 1.1 & 0.4 & 0.2 & 0.1 & 6.2 \\
\hline Others & 8.8 & 9.6 & 14.3 & 19.2 & 21.9 & 11.0 & 12.4 \\
\hline \multicolumn{8}{|l|}{$\begin{array}{l}\text { IMF repayments and interest } \\
\text { (in percent of total debt- }\end{array}$} \\
\hline $\begin{array}{l}\text { service payments) } \\
\text { (in percent of gross }\end{array}$ & 23.2 & 18.5 & 7.1 & 2.1 & 0.7 & 0.7 & 33.2 \\
\hline international reserves) & 5.2 & 4.1 & 1.7 & 1.0 & 0.4 & 0.2 & 13.5 \\
\hline
\end{tabular}

Sources: Staff estimates based on 1999 debt-stock data at decision point; and submissions by the authorities on subsequent disbursements and debt-servicing.

1/ Debt service presented on a commitment basis and includes payments to Alimenta.

2/ In percent of exports and travel income. 
Table 39. The Gambia: Exchange Rate Indicators, 1990-2004

(Period average)

\begin{tabular}{|c|c|c|c|c|c|}
\hline & $\begin{array}{c}\text { Market Rate: } \\
\text { Dalasis per U.S. Dollar }\end{array}$ & $\begin{array}{c}\text { Market Rate: } \\
\text { Dalasis per SDR }\end{array}$ & $\begin{array}{c}\text { Exchange Rate Index: } \\
\text { Dalasis per SDR } \\
(2000=100)\end{array}$ & $\begin{array}{c}\text { Nominal Effective } \\
\text { Exchange Rate } \\
(2000=100)\end{array}$ & $\begin{array}{c}\text { Real Effective } \\
\text { Exchange Rate } \\
(2000=100)\end{array}$ \\
\hline 1990 & 7.88 & 10.69 & 63.42 & 93.08 & 111.28 \\
\hline 1991 & 8.80 & 12.04 & 71.41 & 89.60 & 106.99 \\
\hline 1992 & 8.89 & 12.52 & 74.22 & 92.21 & 109.32 \\
\hline 1993 & 9.13 & 12.75 & 75.59 & 105.18 & 118.28 \\
\hline 1994 & 9.58 & 13.71 & 81.29 & 110.40 & 109.80 \\
\hline 1995 & 9.55 & 14.48 & 85.87 & 105.39 & 105.81 \\
\hline 1996 & 9.79 & 14.21 & 84.27 & 106.15 & 104.68 \\
\hline 1997 & 10.20 & 14.04 & 83.23 & 110.25 & 109.88 \\
\hline 1998 & 10.64 & 14.44 & 85.61 & 109.05 & 107.54 \\
\hline 1999 & 11.40 & 15.58 & 92.39 & 103.83 & 105.19 \\
\hline 2000 & 12.79 & 16.86 & 100.00 & 100.00 & 100.00 \\
\hline 2001 & 15.69 & 19.97 & 118.42 & 85.90 & 87.77 \\
\hline 2002 & 19.92 & 25.79 & 152.93 & 66.50 & 72.34 \\
\hline 2003 & 27.31 & 38.20 & 226.49 & 41.60 & 52.11 \\
\hline 2004 & 30.03 & 44.50 & 263.90 & 36.22 & 51.96 \\
\hline \multicolumn{6}{|l|}{1998} \\
\hline 1st quarter & 10.54 & 14.18 & 84.10 & 111.89 & 106.82 \\
\hline 2nd quarter & 10.56 & 14.15 & 83.90 & 111.21 & 108.96 \\
\hline 3rd quarter & 10.65 & 14.28 & 84.69 & 109.93 & 110.03 \\
\hline 4 th quarter & 10.82 & 15.16 & 89.89 & 103.18 & 104.35 \\
\hline \multicolumn{6}{|l|}{1999} \\
\hline 1st quarter & 11.08 & 15.31 & 90.81 & 104.22 & 105.65 \\
\hline 2nd quarter & 11.30 & 15.22 & 90.28 & 105.69 & 107.81 \\
\hline 3rd quarter & 11.55 & 15.71 & 93.14 & 103.45 & 104.91 \\
\hline 4th quarter & 11.66 & 16.08 & 95.35 & 101.96 & 103.33 \\
\hline \multicolumn{6}{|l|}{2000} \\
\hline 1st quarter & 11.83 & 16.00 & 94.89 & 103.02 & 104.66 \\
\hline 2nd quarter & 12.40 & 16.45 & 97.53 & 101.77 & 102.26 \\
\hline 3rd quarter & 12.73 & 16.66 & 98.77 & 101.45 & 101.01 \\
\hline 4th quarter & 14.20 & 18.28 & 108.42 & 93.76 & 93.01 \\
\hline \multicolumn{6}{|l|}{2001} \\
\hline 1st quarter & 14.96 & 19.32 & 114.56 & 88.30 & 90.59 \\
\hline 2 nd quarter & 15.38 & 19.38 & 114.94 & 89.04 & 90.40 \\
\hline 3rd quarter & 15.85 & 20.14 & 119.40 & 85.01 & 86.85 \\
\hline 4th quarter & 16.56 & 21.03 & 124.72 & 81.27 & 84.10 \\
\hline \multicolumn{6}{|l|}{2002} \\
\hline 1st quarter & 17.60 & 21.99 & 130.39 & 77.31 & 82.25 \\
\hline 2 nd quarter & 18.66 & 23.85 & 141.40 & 71.00 & 75.77 \\
\hline 3rd quarter & 20.40 & 26.99 & 160.02 & 62.42 & 67.72 \\
\hline 4th quarter & 23.01 & 30.59 & 181.41 & 55.27 & 63.63 \\
\hline \multicolumn{6}{|l|}{2003} \\
\hline 1st quarter & 24.65 & 33.74 & 200.05 & 49.29 & 57.92 \\
\hline 2nd quarter & 27.15 & 37.91 & 224.81 & 43.18 & 53.71 \\
\hline 3rd quarter & 27.53 & 38.27 & 226.95 & 38.53 & 49.62 \\
\hline 4th quarter & 29.90 & 43.04 & 255.23 & 35.40 & 47.18 \\
\hline \multicolumn{6}{|l|}{2004} \\
\hline 1st quarter & 30.08 & 44.82 & 265.77 & 35.86 & 49.22 \\
\hline 2 nd quarter & 30.02 & 43.86 & 260.08 & 36.98 & 52.19 \\
\hline 3rd quarter & 30.04 & 44.05 & 261.19 & 36.64 & 53.32 \\
\hline 4 th quarter & 29.97 & 45.29 & 268.56 & 35.40 & 53.11 \\
\hline
\end{tabular}

Sources: Central Bank of The Gambia; and IMF, International Financial Statistics. 
Table 40. The Gambia: Interbank and Parallel Market Exchange Rates, 1998-2004

(Dalasis per unit of foreign currency, unless otherwise indicated; end of period)

\begin{tabular}{|c|c|c|c|c|c|c|c|c|c|c|}
\hline & & \multicolumn{3}{|c|}{ U.K. Pound Sterling } & \multicolumn{3}{|c|}{ U.S. Dollar } & \multicolumn{3}{|c|}{ CFA Franc 1/ } \\
\hline & & $\begin{array}{l}\text { Interbank } \\
\text { mid-market }\end{array}$ & $\begin{array}{c}\text { Parallel } \\
\text { mid-market }\end{array}$ & $\begin{array}{c}\text { Spread } \\
\text { (percent) }\end{array}$ & $\begin{array}{l}\text { Interbank } \\
\text { mid-market }\end{array}$ & $\begin{array}{c}\text { Parallel } \\
\text { mid-market }\end{array}$ & $\begin{array}{c}\text { Spread } \\
\text { (percent) }\end{array}$ & $\begin{array}{l}\text { Interbank } \\
\text { mid-market }\end{array}$ & $\begin{array}{c}\text { Parallel } \\
\text { mid-market }\end{array}$ & $\begin{array}{c}\text { Spread } \\
\text { (percent) }\end{array}$ \\
\hline \multirow[t]{4}{*}{1998} & March & 16.84 & 17.53 & 4.10 & 10.53 & 10.87 & 3.23 & 88.76 & 89.25 & 0.55 \\
\hline & June & 17.00 & 17.53 & 3.12 & 10.59 & 10.87 & 2.64 & 87.51 & 89.25 & 1.99 \\
\hline & September & 17.08 & 17.53 & 2.63 & 10.68 & 10.87 & 1.78 & 91.20 & 89.25 & -2.14 \\
\hline & December & 17.35 & 18.53 & 6.80 & 10.99 & 11.45 & 4.17 & 95.55 & 99.50 & 4.13 \\
\hline \multirow[t]{4}{*}{1999} & March & 17.85 & 18.40 & 3.09 & 11.07 & 11.45 & 3.41 & 93.37 & 95.50 & 2.29 \\
\hline & June & 18.12 & 19.40 & 7.06 & 11.30 & 12.30 & 8.84 & 94.42 & 95.50 & 1.14 \\
\hline & September & 17.96 & 19.18 & 6.81 & 11.66 & 12.33 & 5.76 & 95.22 & 94.00 & -1.28 \\
\hline & December & 18.65 & 19.20 & 2.96 & 11.55 & 12.20 & 5.65 & 92.91 & 94.50 & 1.71 \\
\hline \multirow[t]{4}{*}{2000} & March & 18.58 & 20.55 & 10.58 & 12.14 & 13.20 & 8.74 & 94.92 & 94.50 & -0.44 \\
\hline & June & 19.53 & 20.78 & 6.38 & 12.65 & 13.80 & 9.11 & 94.74 & 97.50 & 2.91 \\
\hline & September & 20.91 & 21.88 & 4.62 & 13.52 & 15.05 & 11.31 & 95.48 & 100.00 & 4.73 \\
\hline & December & 21.09 & 22.40 & 6.19 & 14.89 & 15.55 & 4.45 & 95.50 & 106.50 & 11.52 \\
\hline \multirow[t]{4}{*}{2001} & March & 21.57 & 22.20 & 2.93 & 15.14 & 15.14 & 0.02 & 97.41 & 106.50 & 9.33 \\
\hline & June & 21.44 & 23.40 & 9.12 & 15.50 & 16.75 & 8.05 & 105.84 & 108.50 & 2.52 \\
\hline & September & 21.83 & 24.48 & 12.12 & 16.25 & 17.05 & 4.92 & 106.63 & 117.50 & 10.19 \\
\hline & December & 25.01 & 25.55 & 2.17 & 16.93 & 18.13 & 7.05 & 120.89 & 120.50 & -0.33 \\
\hline \multirow[t]{12}{*}{2002} & January & 25.15 & 25.65 & 1.99 & 17.30 & 18.45 & 6.62 & 115.25 & 120.50 & 4.55 \\
\hline & February & 25.46 & 27.05 & 6.25 & 17.69 & 19.08 & 7.83 & 122.84 & 126.50 & 2.98 \\
\hline & March & 25.81 & 27.20 & 5.39 & 17.82 & 19.23 & 7.89 & 120.36 & 126.50 & 5.10 \\
\hline & April & 26.51 & 27.23 & 2.71 & 18.72 & 19.15 & 2.25 & 125.97 & 125.95 & -0.01 \\
\hline & May & 26.92 & 28.12 & 4.44 & 18.84 & 19.48 & 3.35 & 129.49 & 132.30 & 2.17 \\
\hline & June & 27.40 & 28.92 & 5.52 & 19.14 & 19.68 & 2.82 & 124.23 & 134.40 & 8.19 \\
\hline & July & 28.19 & 31.34 & 11.14 & 19.26 & 20.49 & 6.36 & 130.15 & 150.50 & 15.64 \\
\hline & August & 30.67 & 32.47 & 5.86 & 21.16 & 22.35 & 5.61 & 142.58 & 155.90 & 9.34 \\
\hline & September & 32.60 & 36.13 & 10.80 & 21.79 & 23.70 & 8.77 & 168.53 & 172.00 & 2.06 \\
\hline & October & 34.90 & 36.55 & 4.73 & 22.76 & 23.75 & 4.35 & 170.95 & 175.50 & 2.66 \\
\hline & November & 35.23 & 37.05 & 5.16 & 23.21 & 23.75 & 2.31 & 170.21 & 179.50 & 5.46 \\
\hline & December & 35.49 & 38.13 & 7.43 & 23.39 & 24.75 & 5.80 & 174.45 & 187.75 & 7.62 \\
\hline \multirow[t]{12}{*}{2003} & January & 37.79 & 39.15 & 3.60 & 24.29 & 24.65 & 1.50 & 186.79 & 191.50 & 2.52 \\
\hline & February & 38.69 & 40.20 & 3.91 & 24.63 & 27.00 & 9.61 & 193.40 & 204.00 & 5.48 \\
\hline & March & 40.00 & 42.35 & 5.88 & 26.00 & 27.75 & 6.71 & 205.24 & 221.00 & 7.68 \\
\hline & April & 41.06 & 42.93 & 4.54 & 26.75 & 27.78 & 3.84 & 201.43 & 224.50 & 11.46 \\
\hline & May & 42.40 & 45.13 & 6.44 & 27.19 & 28.10 & 3.36 & 229.81 & 239.50 & 4.22 \\
\hline & June & 44.79 & $\ldots$ & $\ldots$ & 27.88 & $\ldots$ & $\ldots$ & 218.94 & $\ldots$ & $\ldots$ \\
\hline & July & 47.39 & $\ldots$ & $\ldots$ & 29.52 & $\ldots$ & $\ldots$ & 243.51 & $\ldots$ & $\ldots$ \\
\hline & August & 48.77 & $\ldots$ & $\ldots$ & 31.49 & $\ldots$ & $\ldots$ & 255.48 & $\ldots$ & $\ldots$ \\
\hline & September & 53.01 & $\ldots$ & $\ldots$ & 33.43 & $\ldots$ & $\ldots$ & 273.57 & $\ldots$ & $\ldots$ \\
\hline & October & 50.98 & $\ldots$ & $\ldots$ & 31.61 & $\ldots$ & $\ldots$ & 233.13 & $\ldots$ & $\ldots$ \\
\hline & November & 51.29 & $\ldots$ & $\ldots$ & 31.07 & $\ldots$ & $\ldots$ & 242.17 & $\ldots$ & $\ldots$ \\
\hline & December & 51.91 & $\cdots$ & $\ldots$ & 30.96 & $\cdots$ & $\ldots$ & 220.19 & $\cdots$ & $\cdots$ \\
\hline \multirow[t]{13}{*}{2004} & January & 53.27 & $\ldots$ & $\ldots$ & 30.12 & $\ldots$ & $\ldots$ & 248.82 & $\ldots$ & $\ldots$ \\
\hline & February & 53.75 & $\ldots$ & $\ldots$ & 29.83 & $\ldots$ & $\ldots$ & 232.85 & $\ldots$ & $\ldots$ \\
\hline & March & 53.71 & $\ldots$ & $\ldots$ & 30.11 & $\ldots$ & $\ldots$ & 265.34 & $\ldots$ & $\ldots$ \\
\hline & April & 53.69 & $\ldots$ & $\ldots$ & 30.02 & $\ldots$ & $\ldots$ & 271.45 & $\ldots$ & $\ldots$ \\
\hline & May & 53.98 & $\ldots$ & $\ldots$ & 29.99 & $\ldots$ & $\ldots$ & 260.38 & $\ldots$ & $\ldots$ \\
\hline & June & 53.79 & $\ldots$ & $\ldots$ & 30.06 & $\ldots$ & $\ldots$ & 260.86 & $\ldots$ & $\ldots$ \\
\hline & July & 54.32 & $\ldots$ & $\ldots$ & 29.95 & $\ldots$ & $\ldots$ & 262.33 & $\ldots$ & $\ldots$ \\
\hline & August & 54.35 & $\ldots$ & $\ldots$ & 30.02 & $\ldots$ & $\ldots$ & 271.52 & $\ldots$ & $\ldots$ \\
\hline & September & 54.08 & $\ldots$ & $\ldots$ & 30.02 & $\ldots$ & $\ldots$ & 269.72 & $\ldots$ & $\ldots$ \\
\hline & October & 54.43 & $\ldots$ & $\ldots$ & 30.03 & $\ldots$ & $\ldots$ & 268.05 & $\ldots$ & $\ldots$ \\
\hline & November & 54.28 & $\ldots$ & $\ldots$ & 29.96 & $\ldots$ & $\ldots$ & 265.32 & $\ldots$ & $\ldots$ \\
\hline & December & 54.67 & $\ldots$ & $\ldots$ & 29.67 & $\ldots$ & $\ldots$ & 281.83 & $\ldots$ & $\ldots$ \\
\hline & & & $\ldots$ & $\ldots$ & & $\ldots$ & $\ldots$ & & $\ldots$ & $\ldots$ \\
\hline \multirow[t]{3}{*}{2005} & January & 54.61 & $\ldots$ & $\ldots$ & 29.63 & $\ldots$ & $\ldots$ & 277.25 & $\ldots$ & $\ldots$ \\
\hline & February & 54.54 & $\ldots$ & $\ldots$ & 29.49 & $\ldots$ & $\ldots$ & 275.86 & $\ldots$ & $\ldots$ \\
\hline & March & 54.32 & $\ldots$ & $\ldots$ & 29.22 & $\ldots$ & $\ldots$ & 285.21 & $\ldots$ & $\ldots$ \\
\hline
\end{tabular}

Source: Gambian authorities.

1/ Dalasis per CFAF 5,000. 


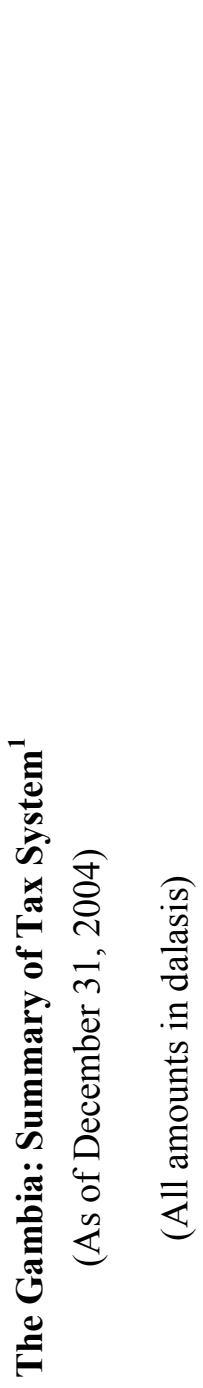

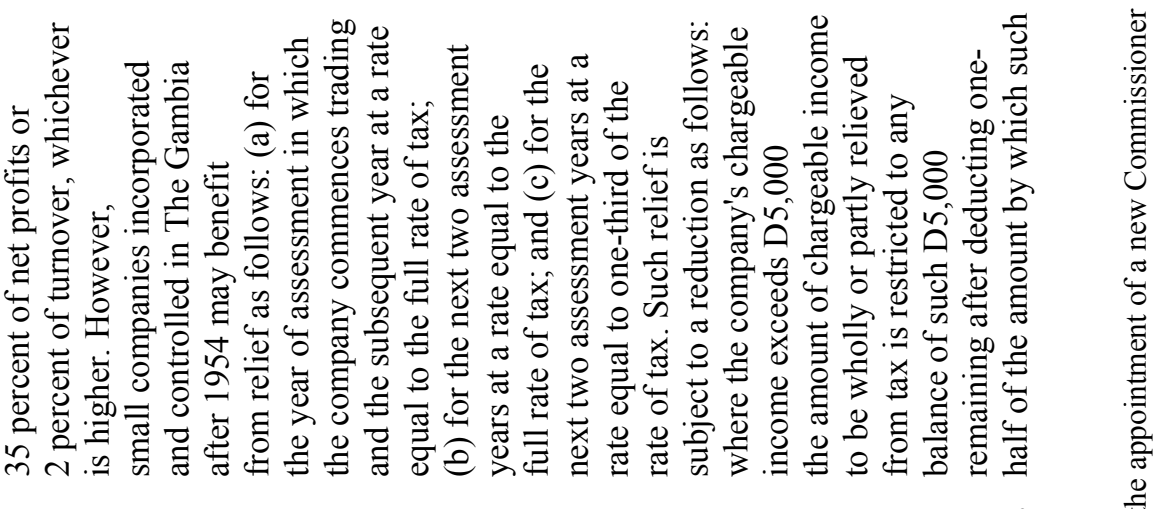

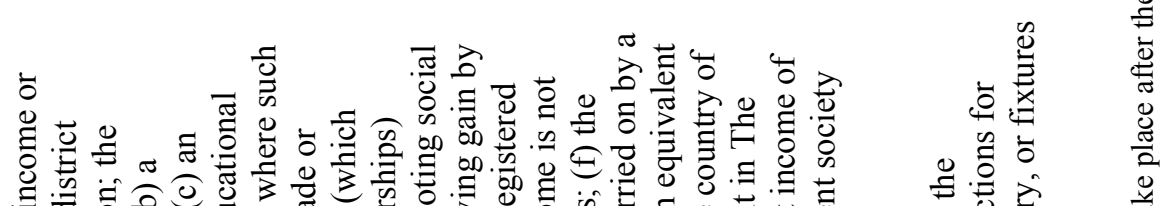

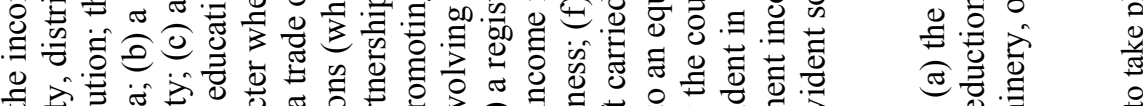

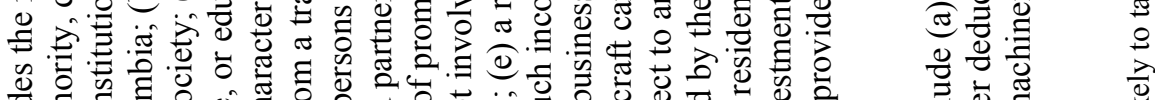

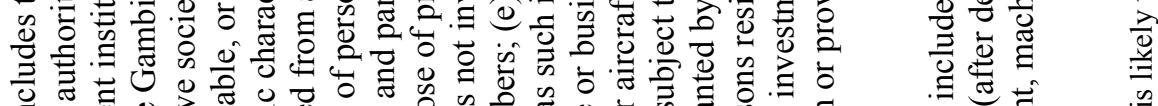

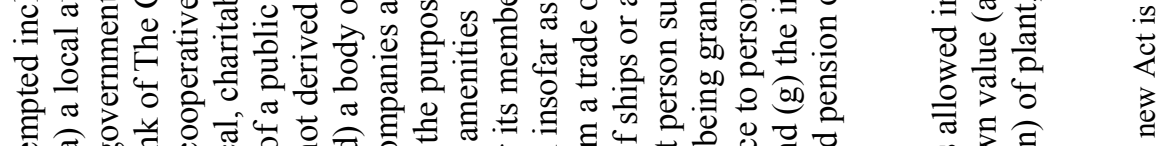
শ

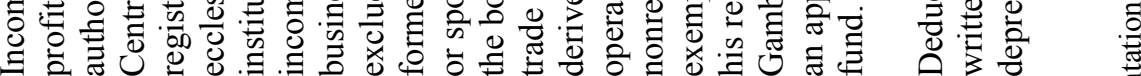

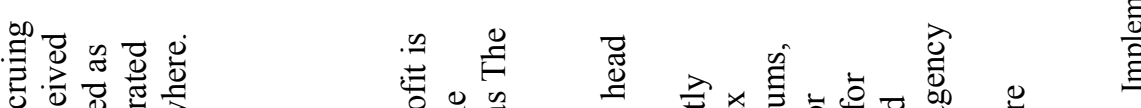

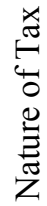

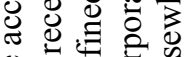
छั



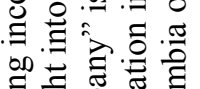

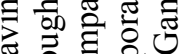
ฮิ

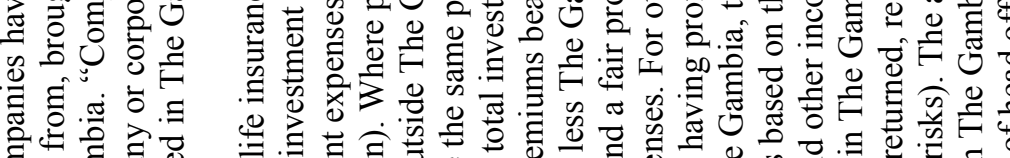
छ ○.

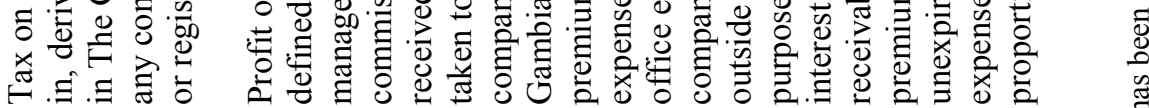

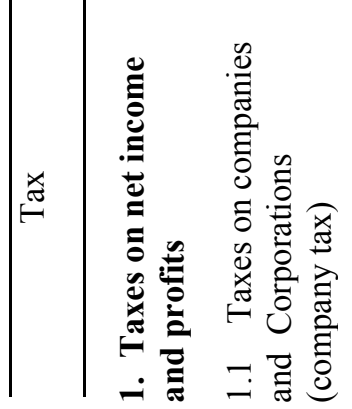




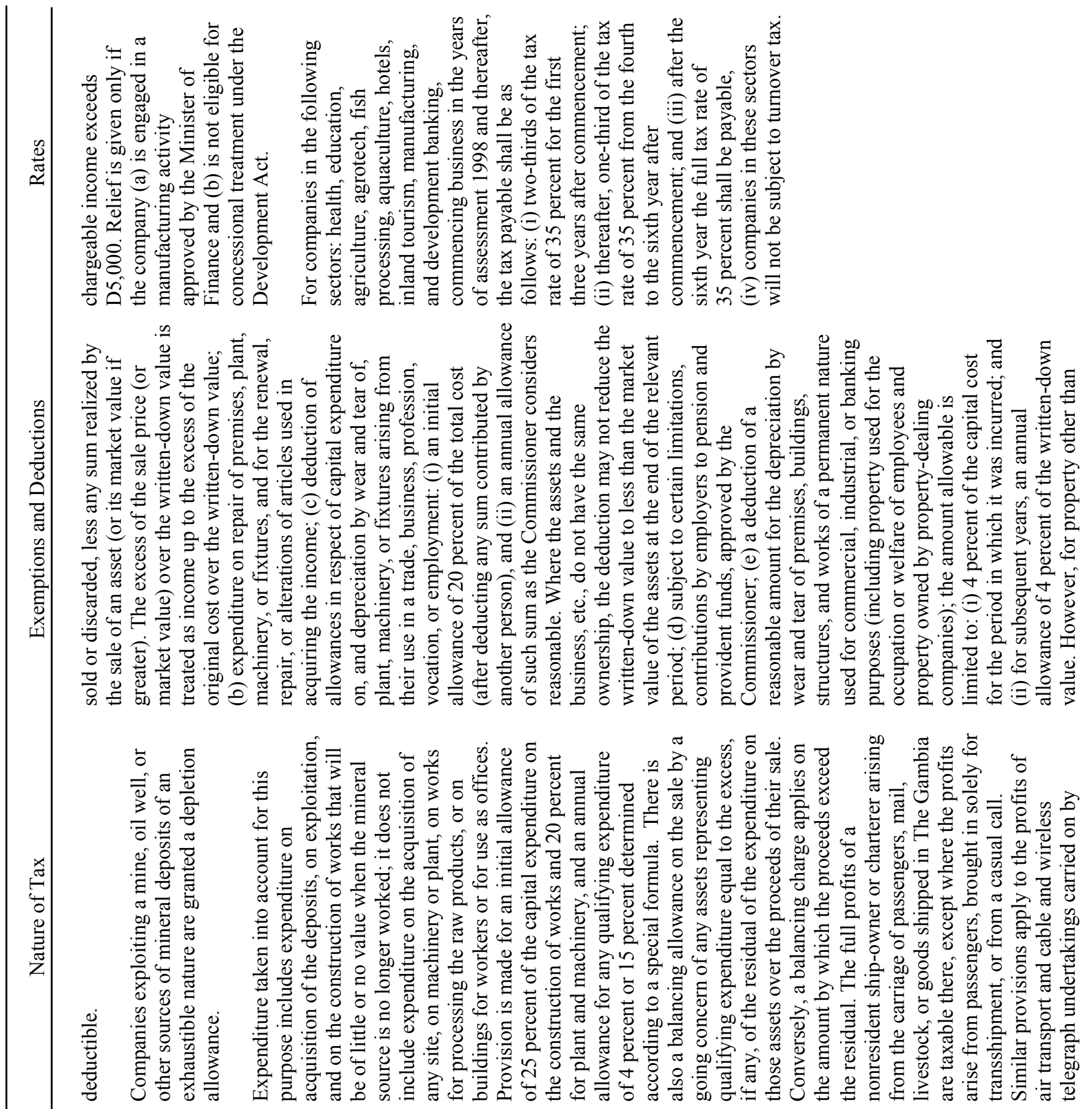




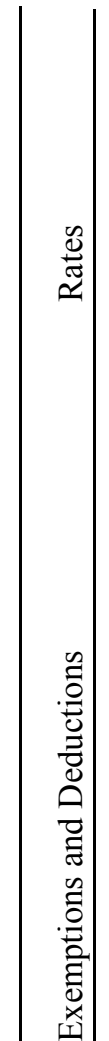

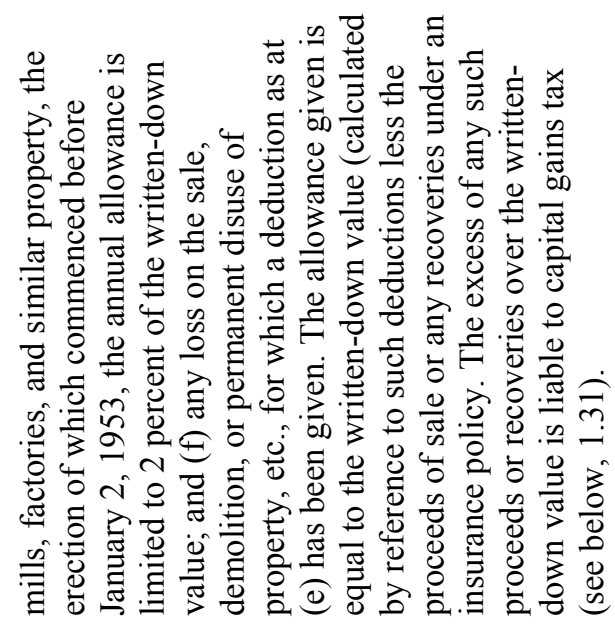

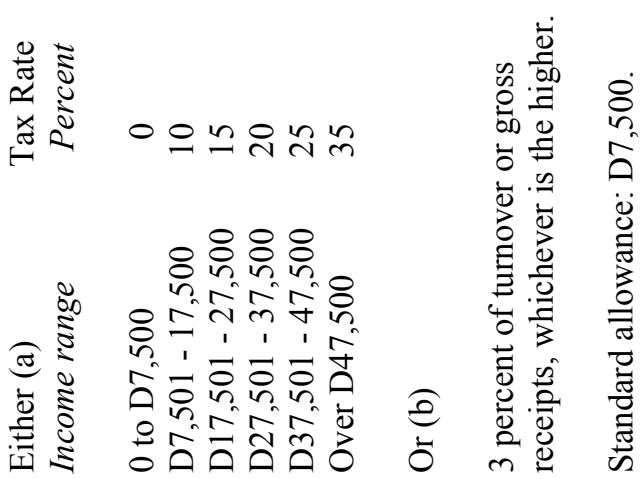

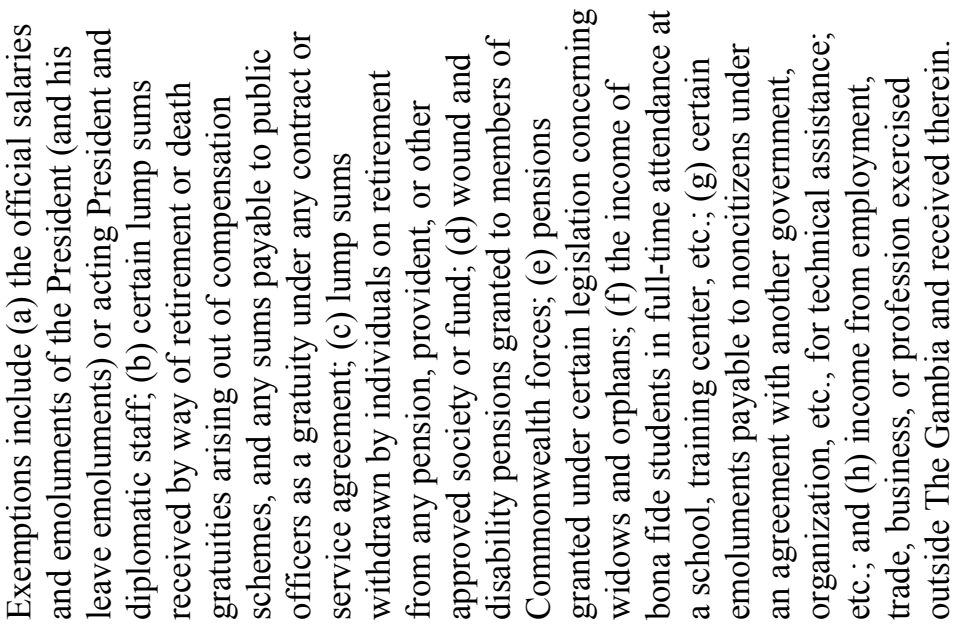
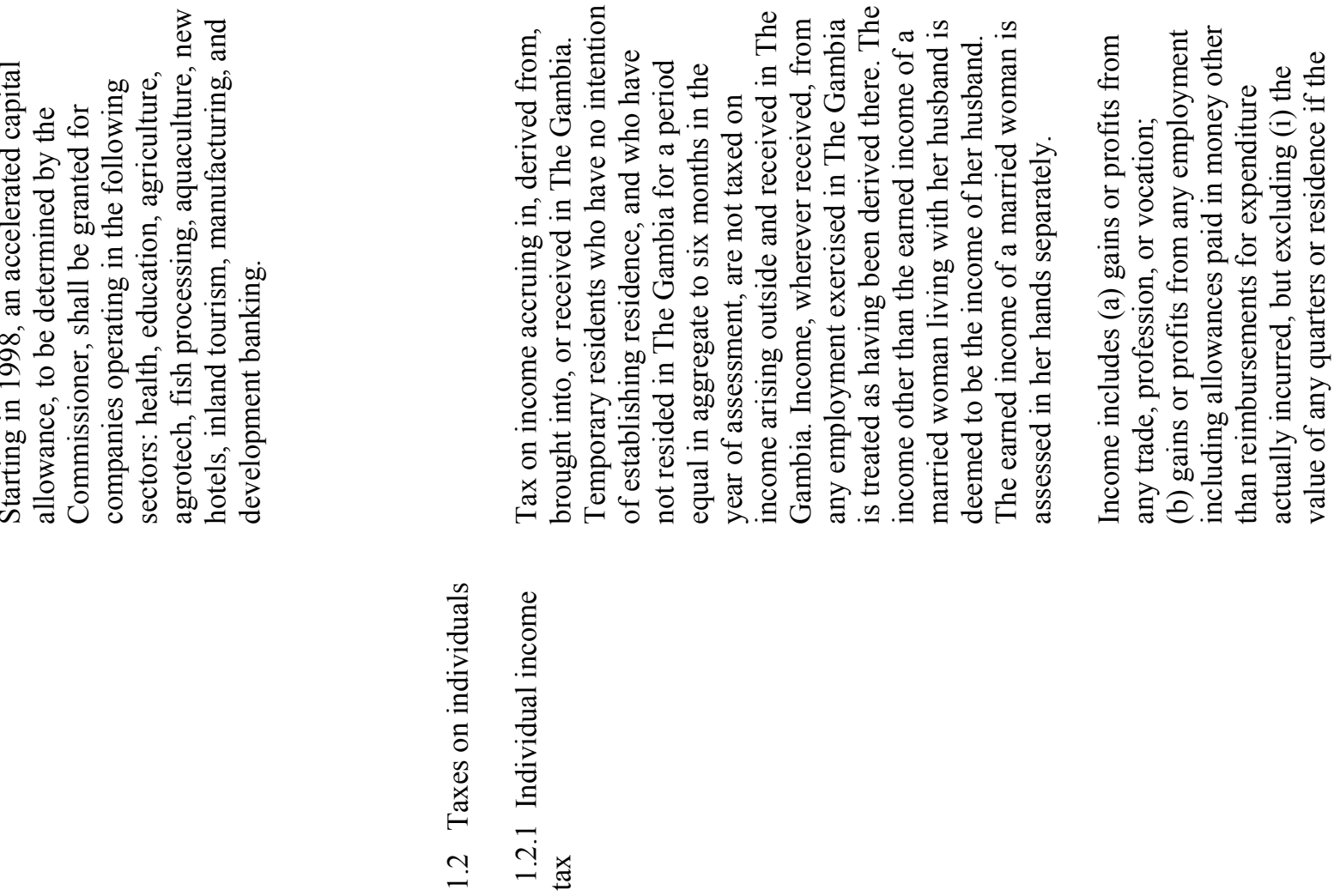


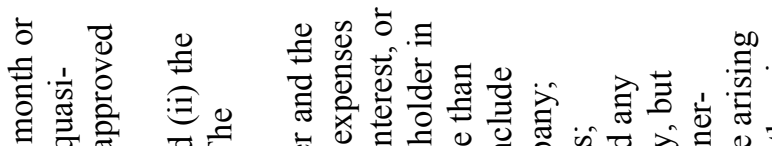

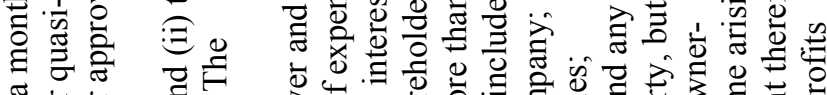

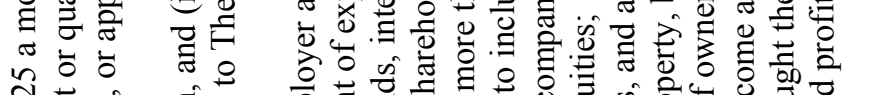




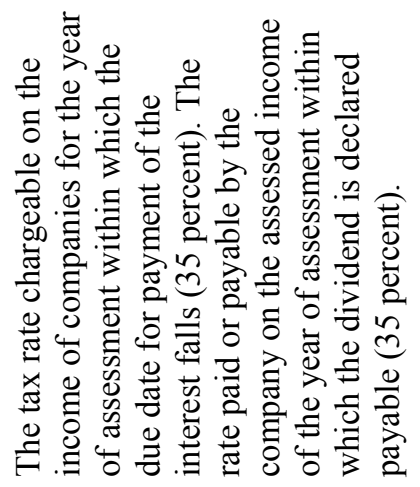

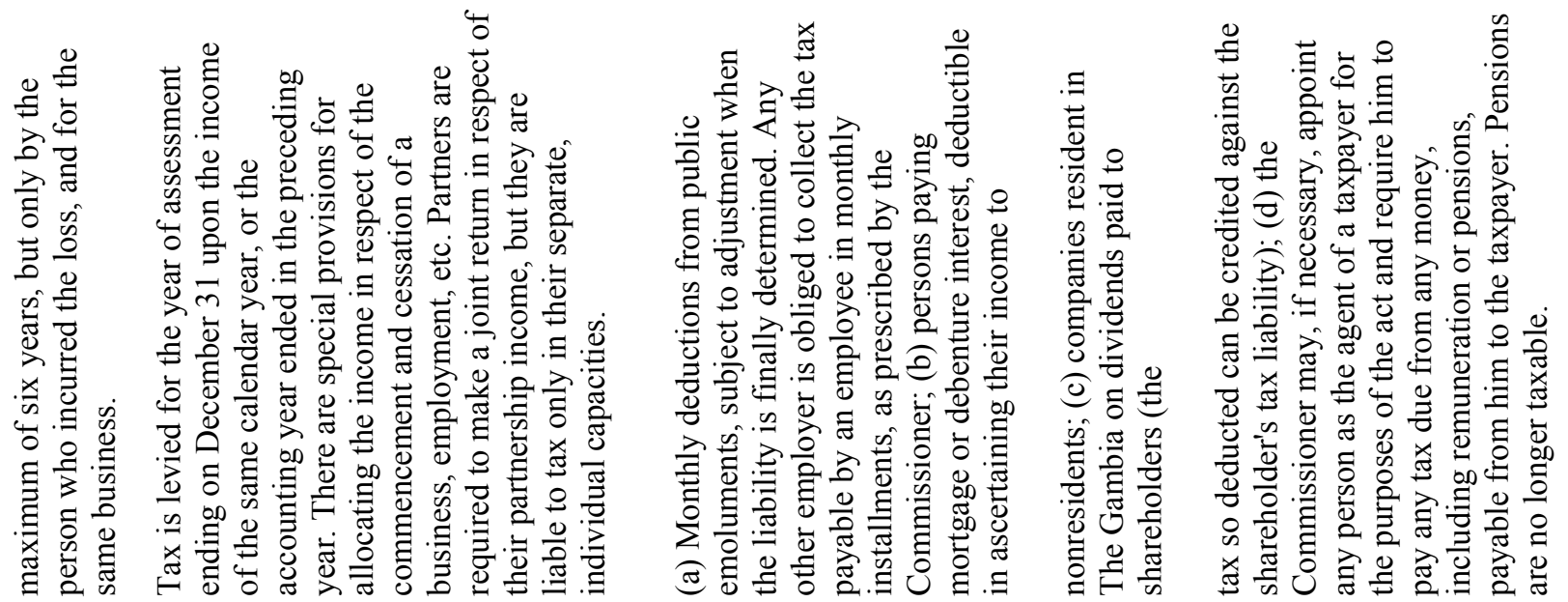

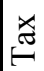

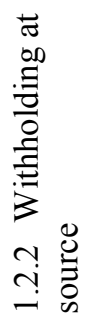




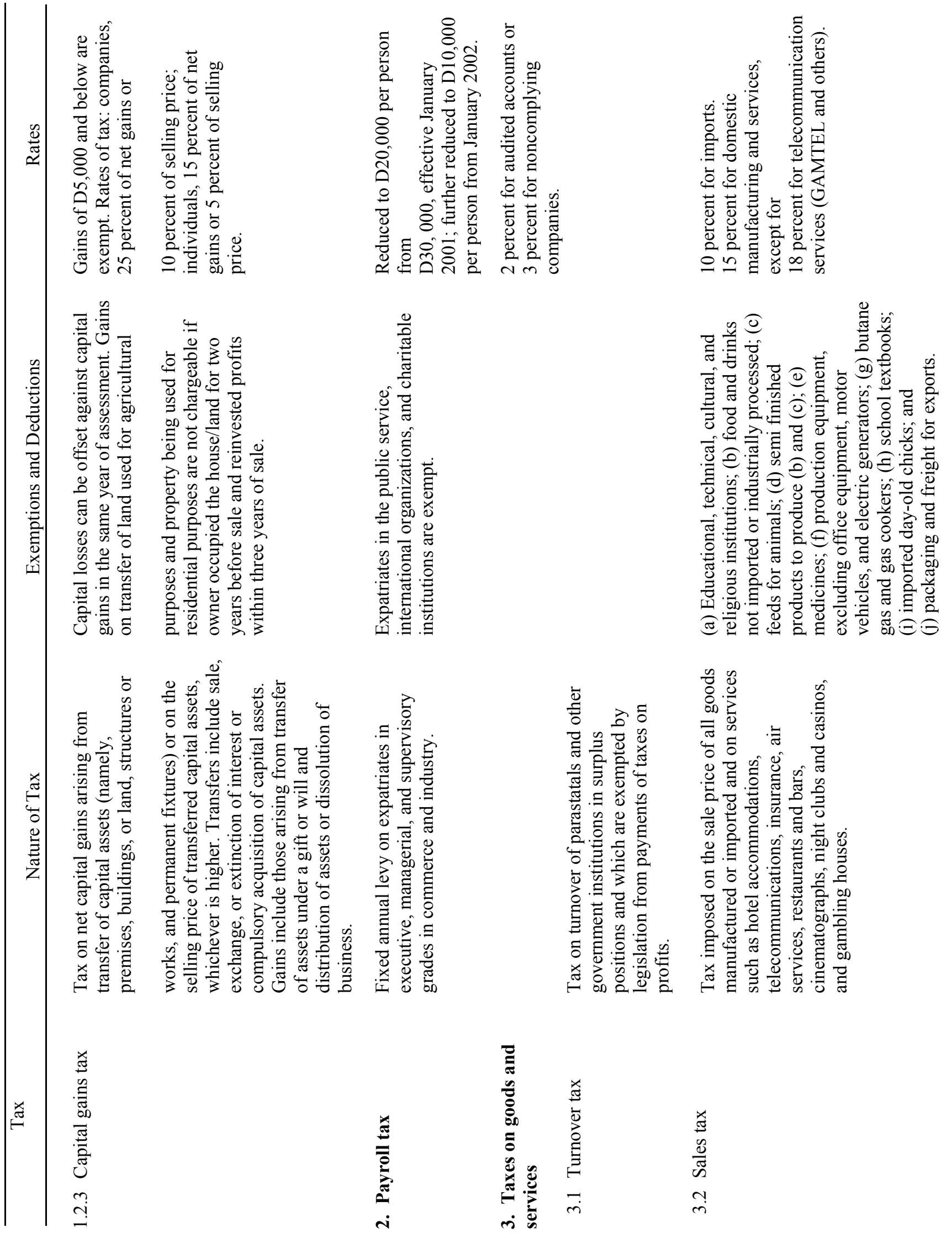




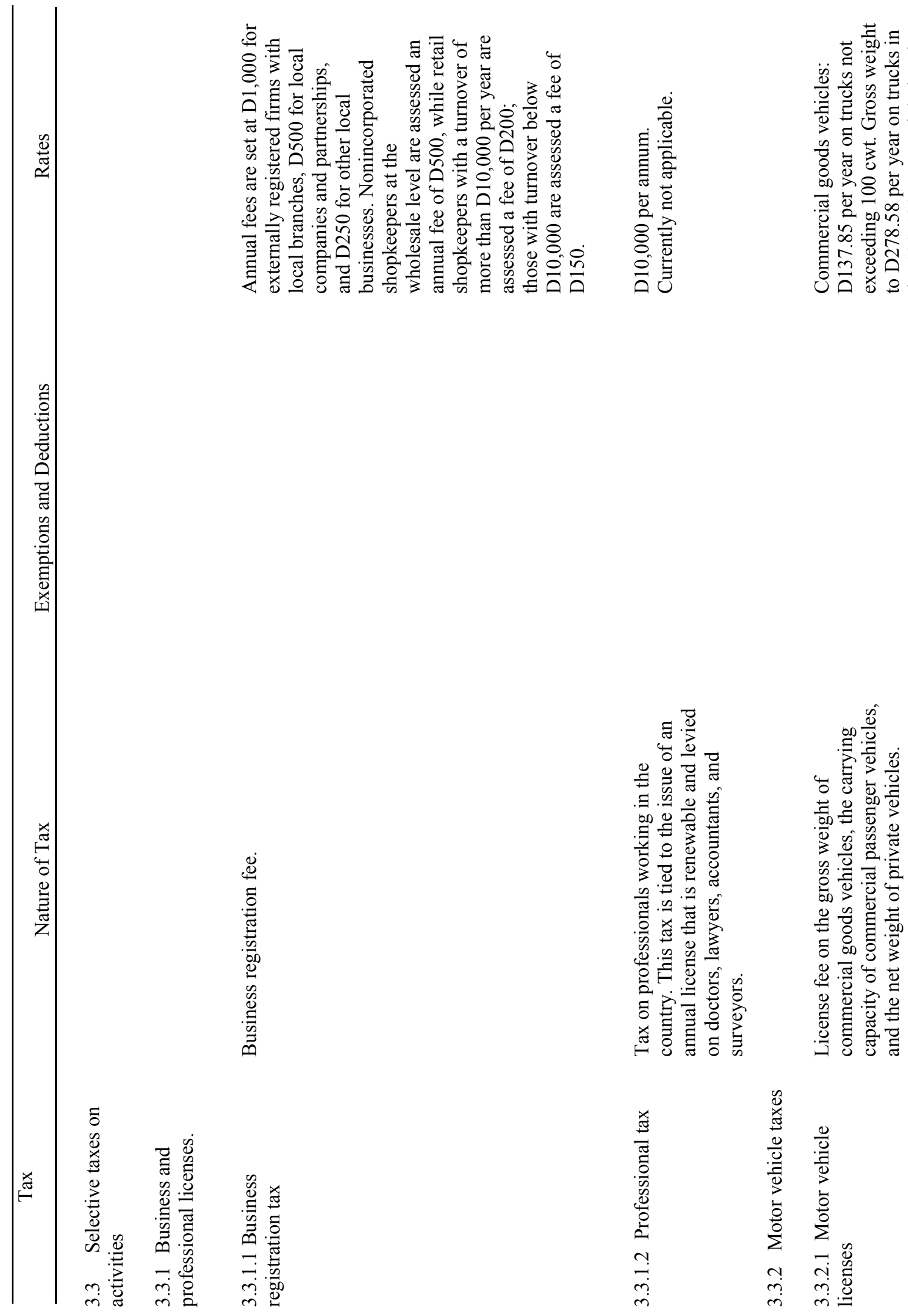




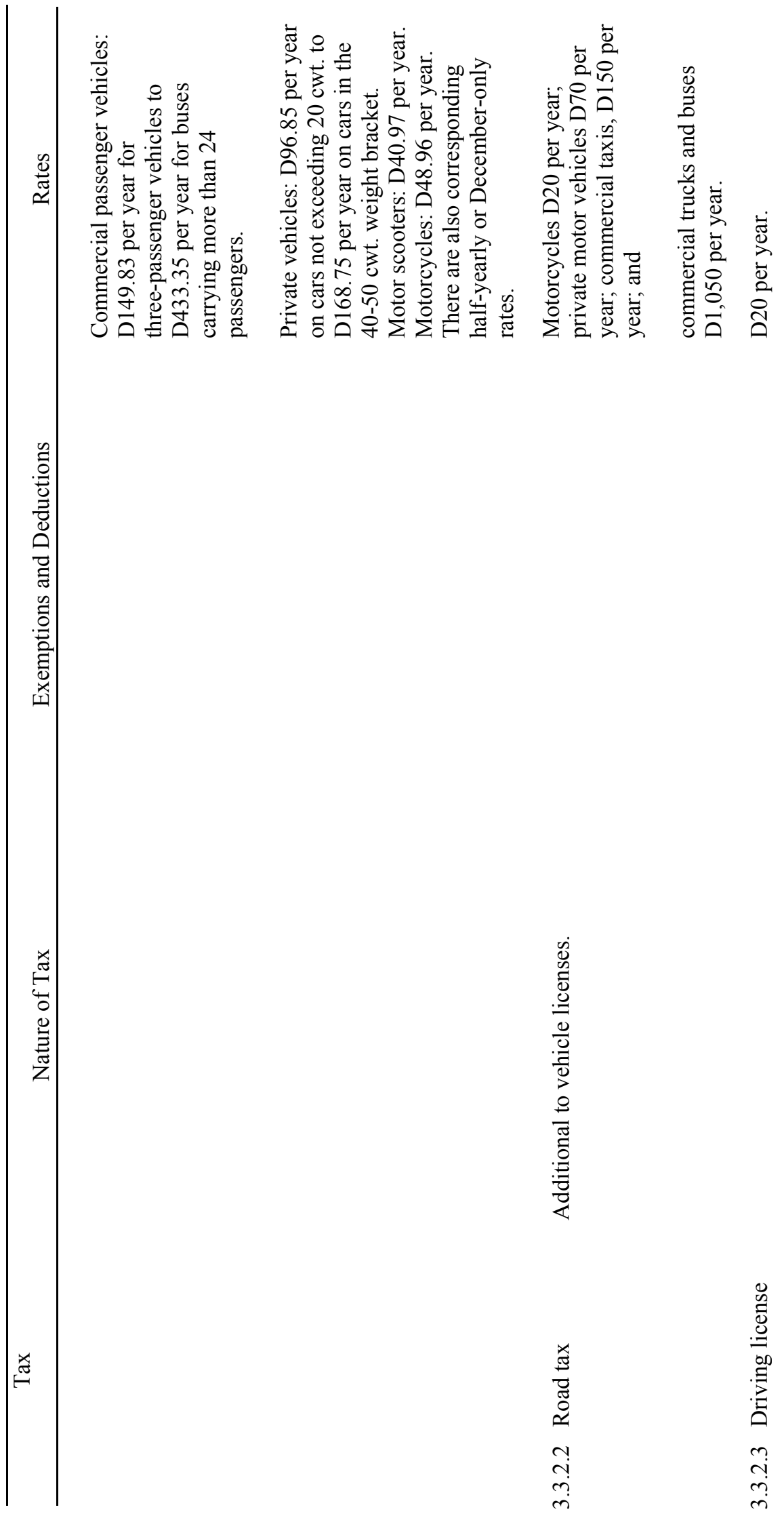




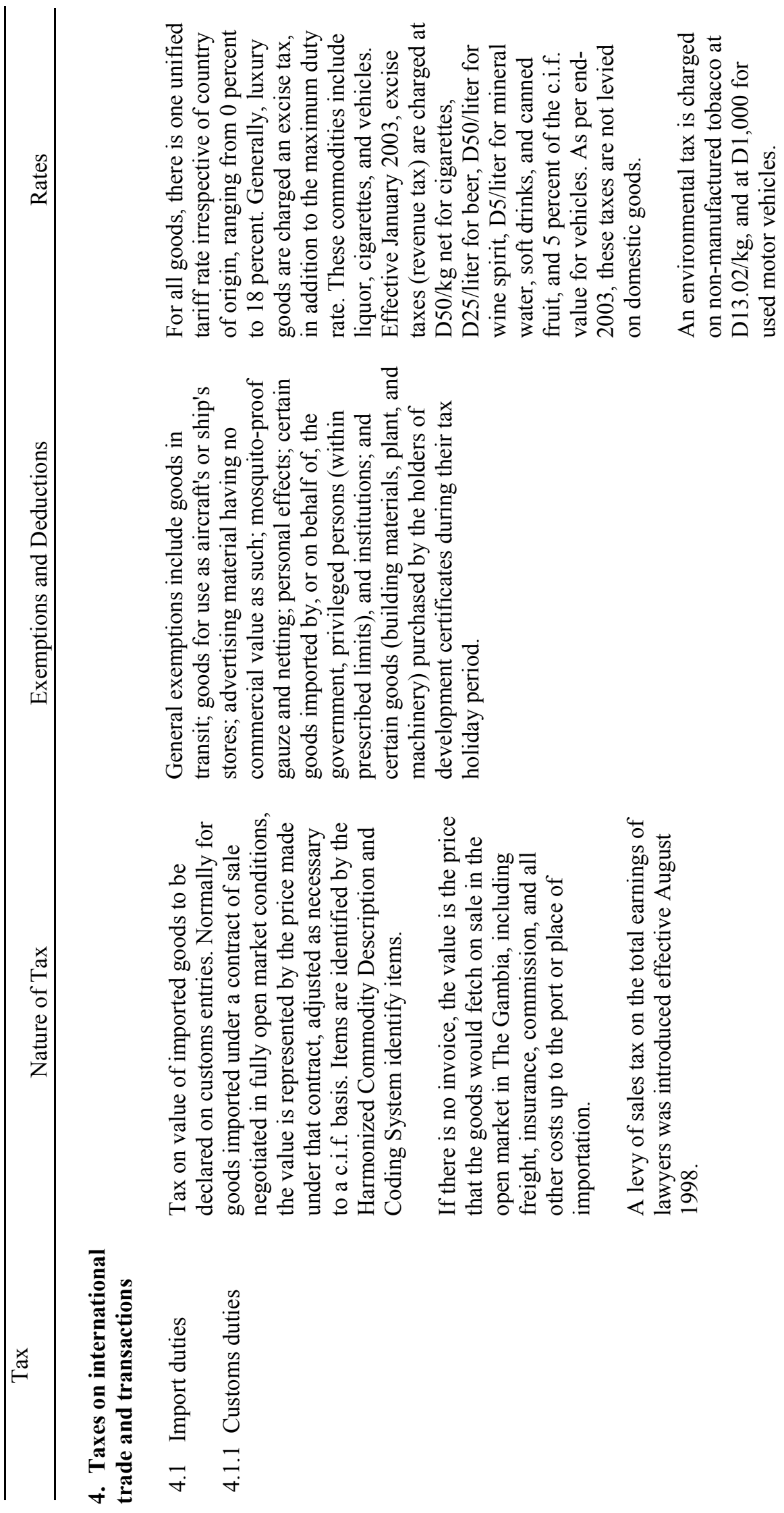




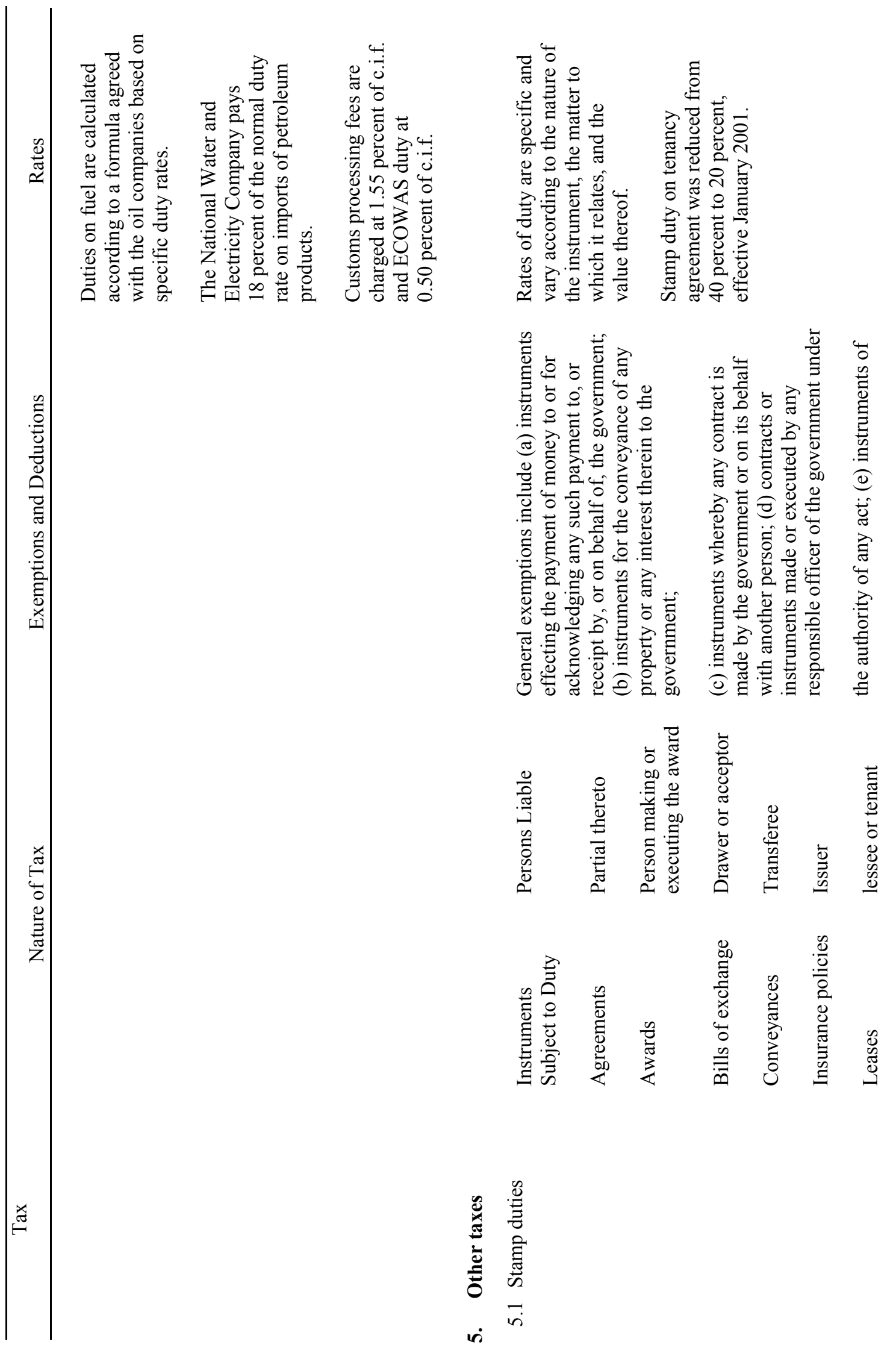




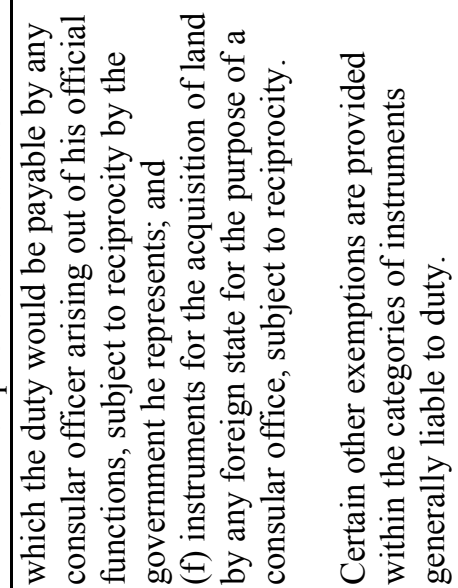

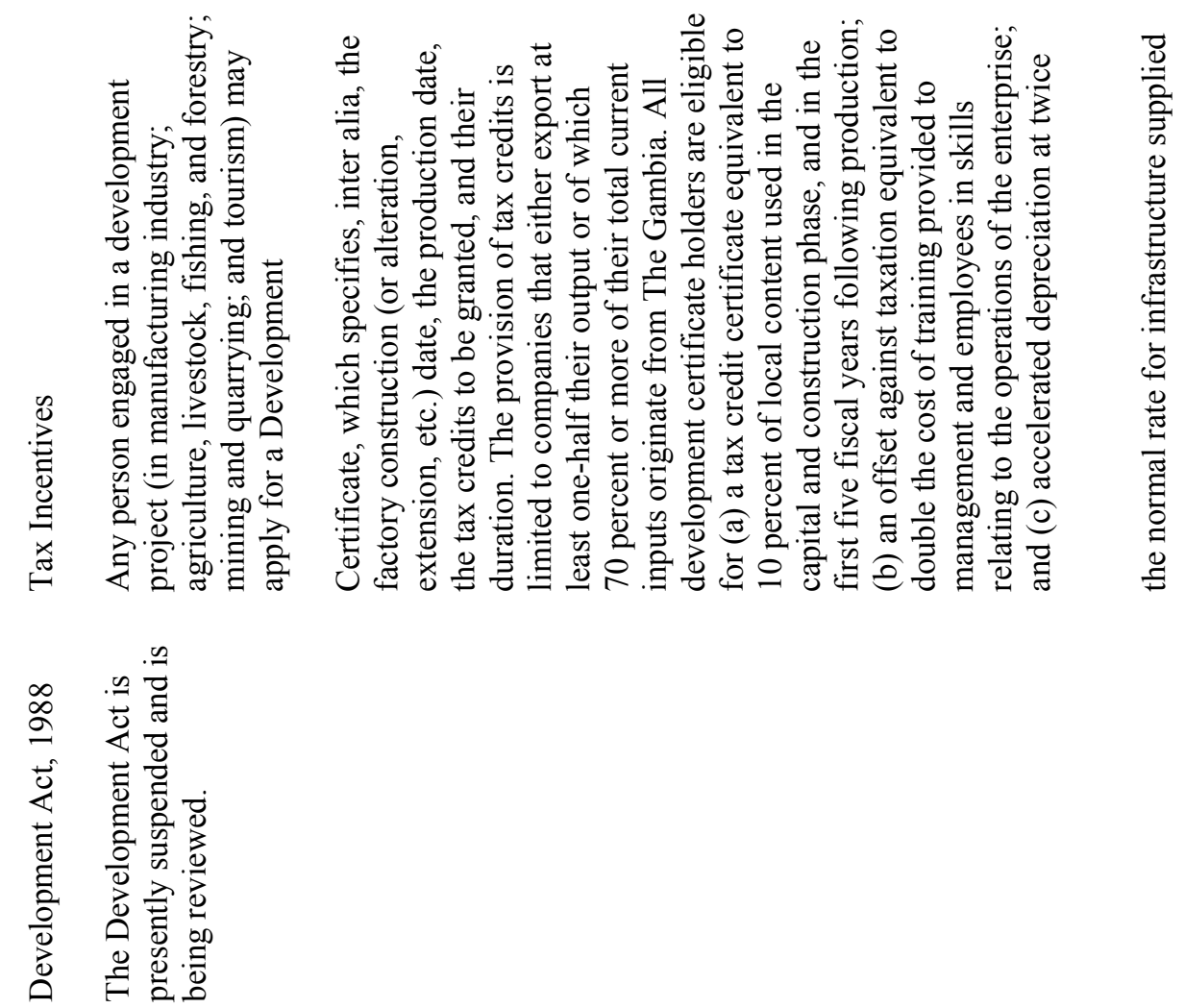




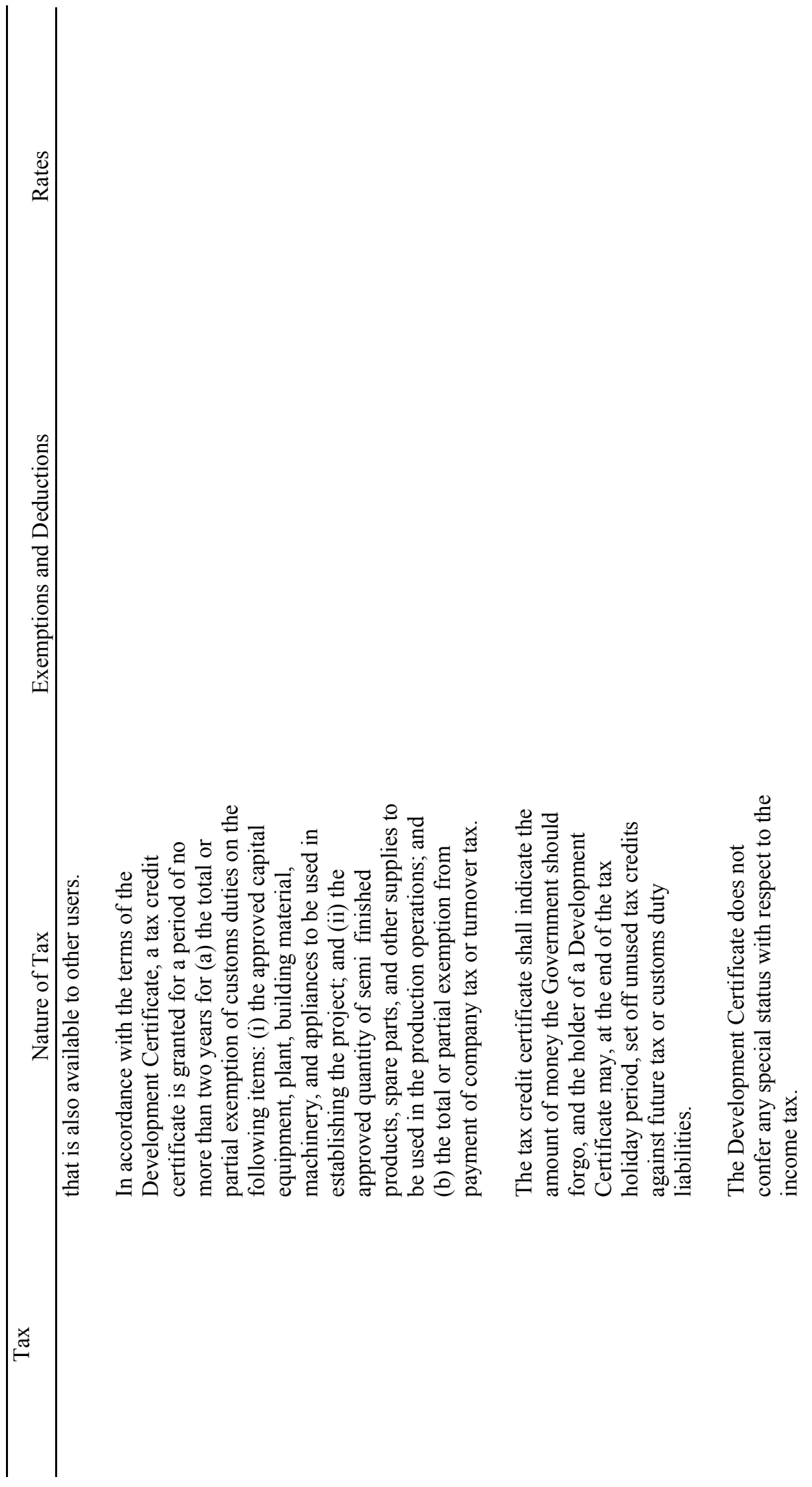

